\title{
TRACK 2: CATHETER INTERVENTIONS FROM FETUS TO ADULT
}

\author{
Abstract no: 23 \\ Device closures of large tubular patent ductus arteriosus with severe pulmonary hypertension: \\ Analysis of 47 cases \\ Nurun Nahar and Fatema Begum \\ Combined Military Hospital, Dhaka, Bangladesh
}

Objective: Large patent ductus arteriosus can cause severe pulmonary hypertension (PHT) due to increased blood flow or increased pulmonary vascular resistance (PVR). Surgical ligations of these large tortuous hypertensive ducts are very difficult. Device closure is an alternative option and here we report 47 such cases which have undergone device closure in our centre.

Patients and methods: A total of 47 patients had large tubular PDA with severe PHT from January 2009 - December 20 I0. Retrospective review of data was done from records kept in the department.

Results: The median age of the patient was 4.5 years (2 months - 45 years) and median weight 9.7kg (3.5 - 60kg). Narrowest PDA diameter varied from 3.4 I $4 \mathrm{~mm}$ with median of $4.8 \mathrm{~mm}$. Twenty nine patients were female, I 8 were male. Pulmonary-to-systemic blood flow ranged from I. I:I - 6.7: I. Pulmonary vascular resistance ranged from 4.2 - 8.I Wood units. Pulmonary arterial pressure ranged from 60/45 (50) - 120/84 (97) mmHg. Systemic blood pressure ranged from 65/40 (48) - $120 / 88$ (99) mmHg. With 100\% oxygen, pulmonary arterial pressure was reduced in 26 cases and remained the same in 9 . In 12 cases device were implanted without this test. Minimum PDA device size was $5 / 4 \mathrm{~mm}$ ADO II and maximum device size was I $8 \mathrm{~mm}$ Amplatzer septal occluder. Fourty three patients had complete occlusion and 4 had residual shunt. 3 cases had complete occlusion within Ist month. One patient developed haemolysis and managed with coil implantation. Embolisation was experienced in I case. On echocardiography, RV pressure reduced to normal by 3 months in all the cases except 2. Conclusion: Tubular PDA associated with severe PHT could be successfully closed by ductal occluders. After occlusion, pulmonary pressure had definite reduction and result in our centre is encouraging.

\section{Abstract no: 39 \\ Impact of fenestration creation on managing patients with protein losing enteropathy complicating Fontan procedure}

\section{Mashail Binobaidan and Jasim Abdulhameed}

Prince Sultan Cardiac Centre, Armed Force Hospital, Riyadh, Saudi Arabia

Introduction: Protein losing enteropathy (PLE) is a well known complication following Fontan procedure and one way of managing it is to create fenestration if it is not present, or by enlarging if it is small to reduce the Fontan pressure and reduce venous congestion which results in intestinal protein loss.

Aim: To evaluate the effect of such a procedure in our population.

Method and material: From February 2006 - October 20II, 9 patients - who had fenestration creation due to the development of PLE - were assessed with regard to clinical, laboratory result and haemodynamic effect pre- and post-procedure. Median age 7 years (4 - 21 ), median weight $23 \mathrm{~kg}$ (I 5 - 52 ); male: female ratio 3.5:I (male and 2 female); median saturation pre- and post procedure was $93 \%$ and $82 \%$ respectively; median albumin pre- and post-procedure was I8gm/ $\mathrm{dl}$ and $3 \mathrm{Igm} / \mathrm{dl}$ during the first 2 weeks and $36 \mathrm{gm} / \mathrm{dl}$ thereafter; median pulmonary artery pressure before and after was $25 \mathrm{mmHg}(\mathrm{I} 7 \mathrm{-} 32 \mathrm{mmHg}$ and $16 \mathrm{mmHg}$. Results: (14 - 19mmHg) transpulmonary pressure gradient reduced from a median of I I mmHg - $5 \mathrm{mmHg}$; No immediate deaths; 2 patients needed re-dilatation; there were 2 (22\%) late deaths; ( I patient had stent thrombosis I month after surgery followed by fulminant pulmonary embolism, though all patients were on anti-coagulant; the other I came to the emergency unit with sudden arrest but could not be resuscitated); 3 (33\%) patients have persistent low albumin though the fenestration is patent.

Conclusion: Transcatheter fenestration creation to manage PLE following the Fontan procedure is feasible and can be done in the cath lab with little morbidity and mortality and with beneficial effect; however late complications and completely solving PLE is of concern, especially if done late.

\author{
Abstract no: 40 \\ Right ventricular outflow tract stenting in the symptomatic infant with Tetralogy of Fallot \\ Mashail Binobaidan and Jassim Abdulhameed \\ Prince Sultan Cardiac Centre, Armed Force Hospital, Riyadh, Saudi Arabia
}

Background: The debate continues regarding the initial management of cyanotic or duct-dependent infants with ToF especially those patients with pulmonary artery hypoplasia. While repair can and has been performed in these patients, it is associated with increased morbidity.

Objective: We review the effectiveness of right ventricular outflow tract (RVOT) stenting in the symptomatic young infant with ToF.

Methods and material: Clinical, echocardiographic, angiographic and haemodynamic data were reviewed for 13 patients who underwent 17 RVOT stenting procedures from March 2008 - January 2012. There were 8 girls and 5 boys with median age 3 months and weight $3.5 \mathrm{~kg}$. The pulmonary valve was hypoplastic in all patients; median pulmonary valve diameter 3.I mm (range 2.7-5.2); and Z-score -5.5 (range -8.9 to -4.4).

Results: RVOT stenting improved arterial oxygen saturation from a median of 60\% (55 - 66\%) - $91 \%$ (82 - $94 \%$ ). Median Z-score for the left pulmonary artery increased from $-4.2(-7.2$ to -2.9$)$ before stent implantation, to $-1.8(-4.6$ to -0.8$)$ at time of surgery. Median Z-score for the right pulmonary artery increased from -3.1 ( -6.2 to -2.1$)--0.5(-2.1$ to 0.2$)$. There were no complications. Nine patients have undergone successful repair. There were no immediate or early deaths.

Conclusions: In the symptomatic young infant with ToF, who abandoned surgery for any reason or at high risk, stenting of the RVOT provides a safe and effective management strategy, improving arterial oxygen saturation and encouraging pulmonary artery growth. 


\title{
Abstract no: 105 \\ First experiences with the GORE $^{\circledR}$ septal occluder in children with atrial septal defects
}

\section{Camilla Nyboe*, Vibeke Elisabeth Hjortdal" and Jens Erik Nielsen-Kudsk*}

"Department of Cardiothoracic Surgery, Aarhus University Hospital, Aarhus, Denmark

"Department of Cardiology, Aarhus University Hospital, Aarhus, Denmark

Background: Transcatheter closure is considered standard treatment for most atrial septal defects (ASD). In this study we report the first experiences with the GORE $^{\circledR}$ septal occluder (GSO) in children with ASDs. The GSO is a new, non-selfcentering, retrievable, two-disc occluder shaped from five Nitinol wires covered by ePTFE. It resembles the GORE ${ }^{\circledR}$ HELEX Occluder but has been improved in terms of septal apposition, tissue ingrowth and delivery. It is a soft occluder with a presumed low risk of erosion available in sizes 15, 20, 25 and 30mm.

Materials and methods: Eleven children with a secundum ASD were selected for GSO closure. Mean age was $7.5 \pm 4.2$ years (4 - 17years) and mean weight $26.6 \pm 13.1 \mathrm{~kg}(16-45 \mathrm{~kg})$. Closure was performed through an IIF sheath in the right femoral vein in general anaesthesia guided by TEE. All defects were balloon sized and device size was chosen according to an occluder/defect size ratio $\geq 2$. TTE was used for follow-up (median 3 months). Mean ASD size was II.2 $\pm 1.7 \mathrm{~mm}$ (7 - I $13 \mathrm{~mm})$ and GSO size was $23.2 \pm 3.3 \mathrm{~mm}$ (I5-25mm). Three patients had I - 3 additional defects. Eight patients had a deficient aortic rim and I patient had a septal aneurysm. Seven patients had symptoms before closure and all had dilated right ventricles.

Results: Implantation was successful in all patients with no complications. Delivery was easy and intuitive by the new delivery system. No patients had residual shunts at follow-up. Mean procedure time was $37 \pm$ I I minutes (20 - 55 minutes) and flouro time was I I \pm 5.3 minutes (5 - 24 minutes). At follow-up all patients were relieved of symptoms, none claimed of palpitations, all had sinus rhythm and right ventricle dimensions were normalised.

Conclusion: The GORE ${ }^{\circledR}$ septal occluder is an efficient and safe device for closing ASDs in children with defects up to $15 \mathrm{~mm}$. It is feasible for ASDs with a deficient aortic rim and has a presumed low risk of erosion.

\section{Abstract no: I I 3 \\ Transcatheter VSD closure: Comparison of different devices and methods and their success rates}

Hojjat Mortezaeian Langeroudi, Yasaman Khalili, Akbar Shahmohamadi, Mahmoud Meraji, M.Y Aarabi, Avisa Tabib, P.N. Davari and M Vesal Refugee Health Care University of Medical Sciences (TUMS), Tehran, Iran

Background: Ventricular septal defects (VSD) were previously treated surgically. The introduction of percutaneous VSD closure via different devices, the management of PM VSD, muscular VSD and residual VSDs has evolved. In our centre, Amplatzer and coil devices have been implanted for selected PM VSD, muscular VSD and residual post-surgical VSDs since 2006.

Methods and material: The charts of all VSD closures since 2006 were reviewed retrospectively. Clinical, electrocardiographic, and echocardiographic data were analysed. The pre-closure, immediate post-closure and I-month, 6-month, and 12-month post-closure results were assessed. 107 patients (50 male (46.7\%), 57 female (53.3\%)) with mean age $9.40 \pm 4.22$ years ( 0 - 18 years) were evaluated. The mean diameter was $4.69 \pm 1.79 \mathrm{~mm}$ (minimum 2 up to maximum $12 \mathrm{~mm}$ ) and mean PAP patients was $17.98 \pm 6.86 \mathrm{mmHg}(13-70 \mathrm{mmHg})$. The mean ratio QP: Qs $1.4 \pm 0.13$ was calculated respectively (1.3 - 1.8). Nineteen patients (I7.9\%) had LVH before the procedure: 12 patients ( 1 I.2\%) had trace; 5 patients (4.7\%) mild; and II patients ( $10.3 \%$ ) had moderate aortic regurgitation. Sixteen patients ( $15 \%$, had trace regurgitation; 22 patients (20.6\%) had mild; and 7 patients (6.5\%) had moderate tricuspid regurgitation.

Results: The results showed 104 cases were closed successfully; 3 cases failed; a completely closed shunt were detected in $91.6 \%$ of patients ( 98 patients; while shunt remained in 5 patients (4.7\%); and there was a trace tricuspid regurgitation in 4 patients (3.7\%). The time for all arrhythmias onset was in the first 24 hours after the procedure, and right bundle branch block was the most common.

Conclusion: Congenital VSD closure using transcatheter devices resulted in stable improvement in clinical status and decreased interventricular shunting. Transcatheter device closure is an effective management option for patients with complex muscular VSDs that are difficult to approach surgically and for postoperative residual VSDs. To avoid intervention complications such as complete heart block and aortic insufficiency in patients with VSD, still remains a challenge. Selection a suitable device with the appropriate size and shape is the most important factor for success.

\author{
Abstract no: 115 \\ path after birth \\ Mats Mellander*, Oscar Svensson", Annika Ãhman" and Katarina Hanseus ${ }^{\dagger}$ \\ "Sahlgrenska University Hospital, Goteborg, Sweden \\ \#Uppsala University Hospital, Uppsala, Sweden \\ tSkåne University Hospital, Lund, Sweden
}

Outcome after pre-natal diagnosis of cardiac malformations predicted to require a univentricular

Background: Data on the prognosis for children with univentricular (UV) hearts is mainly based on surgical follow-up. However, knowledge of the outcome after fetal diagnosis is necessary during prenatal counselling.

Materials and methods: This 2-centre study analyse the outcome in 105 consecutive fetuses predicted to certainly $(n=79)$ or probably $(n=26)$ require a UVpath. Termination was an option <22 weeks. Median follow-up in survivors was 5.6 years $(0.3-10)$. Diagnoses in survivors were HLHS+variants (I5), TA (6), DILV (3), CoA (3), AVSD (2), PA/IVS (2), VSD thypoplastic RV ( I), complex UV (2) and complex TGA ( I). Survival was 33\% (35/I 05) after fetal diagnosis, $54 \%(35 / 65)$ in continuing pregnancies, 58\%(35/60) after live birth and 73\%(35/48) after surgery. Survival to surgery in continuing pregnancies was $74 \%$ (48/65). Results: The most common diagnoses were: HLHS+variants (37+7): unbalanced AVSD (17): CoA+LV-hypoplasia (9); PA/IVS (8); TA (8); AS (6); and DILV (5); aneuploidy (8); extracardiac malformations (9); isolated cardiac defects (88). $40 / 67$ (60\%) diagnosed $<22$ weeks chose termination. Of the remainder, 5 fetuses died due to AVSD+heart-block, CoA+diaphragmatic hernia, Ebstein+large TR, HLHS+trisomy 18 and PA/IVS+large TR respectively; 60 were live born; 12 died unoperated (inoperable (5); preterm (4), hydrops (I); I had trisomyl 8 and extracardiac malformations (I)). Of the 48 patients who were operated results were; stage I (40); stage I+II (32); stage I-III (20); stage I-III+transplant (I); and biventricular (BV) correction (8=I certain, and 7 probable pre-natal 
prediction of UV). I3/48 operated on died (27\%): between stages I-II 97); after a HLHS-hybrid procedure ( I); after stage II (2); from malignancy after transplant (I); and after BV correction (2). Median age at post-operative death was 3 months (0.2-40). Median follow-up in survivors was 5.6 years (0.3 - I0). Diagnoses in survivors were HLHS+variants (I5); TA (6); DILV (3); CoA (3); AVSD (2); PA/IVS (2); VSD+hypoplastic RV ( I); complex UV (2); and complex TGA (I), Survival was 33\% (35/I05) after fetal diagnosis; 54\% (35/65) in continuing pregnancies; 58\% (35/60) after live birth; and 73\% (35/48) after surgery. Survival to surgery in continuing pregnancies was $74 \%$ (48/65).

Conclusions: $74 \%$ of fetuses in continuing pregnancies survived to have surgery. Medium term surgical survival was $73 \% .60 \%$ chose termination if diagnosed $<22$ weeks. This is useful information in pre-natal counselling.

\author{
Abstract no: 144 \\ Can prenatal intracardiac echogenic foci affect postnatal cardiac function? \\ Rima Bader \\ King Abdul Aziz University Hospital, College of Medicine, Jeddah, Saudi Arabia
}

Objective: To determine whether prenatally diagnosed intracardiac echogenic foci (ICEF) are associated with neonatal cardiac dysfunction and persistence. Methods: Fetuses with ICEF shown on pre-natal sonography (January 2010 - December 20I I) at I peri-natal centre underwent post-natal echocardiography at ages I month - I year. A single peri-natal cardiologist assessed cardiac function by measuring the left ventricular shortening fraction (LVSF) and myocardial performance index (MPI). The presence of tricuspid valve regurgitation (TR) was sought.

Results: Prenatally, 24 fetuses had ICEF, mean age at diagnosis 25 \pm 3 . I weeks. 18 (75\%) fetuses had left ventricular intracardiac echogenic foci (LVIEF) and 6 (25\%) fetuses had right ventricular intracardiac echogenic foci (RVIEF). On examination post-natally I4 (58\%) of the infants were male and I 0 (42\%) female with a mean age of $8.2 \pm 4.1$ months. Pre-natally, all infants had normal LVSF. The overall mean left ventricular MPI (reference value, $0.36 \pm 0.06$ ), was normal for both infants with LVIEF $(0.34 \pm 0.06)$ and RVIEF (0.33 \pm 00.04$)$. Trace TR were noted in 12 (50\%) of the infants. LVIEF persisted in I4 infants (77\%), whereas RVIF persisted in 2 (33\%).

Conclusions: Pre-natally diagnosed ICEF can be persistent but is not associated with myocardial dysfunction in the Ist year of life.

\title{
Abstract no: 145 \\ Transcatheter fenestration in Fontan failure : Why, how an by which result? \\ Dietmar Schranz \\ Paediatric Heart Center, Justus-Liebig University, Giessen, Germany
}

Total Cavo-Pulmonary Connection (TCPC) is the final step of the palliative separation of the circulations in children with a univentricular heart. Fenestration between the systemic venous conduit and the common atrium might be a life saving approach.

Between 2005 and 2011, 22 percutaneous catheter-based fenestrations were performed in 19 patients, in 17 with an extra cardiac conduit, in 2 with a lateral tunnel. A fenestration of a TCPC tunnel was newly created or a surgical closed reopened in II patients each. Seven (36.8\%) had a dominant left ventricle and 12 (63.2\%) a dominant right ventricle. The median time between the TCPC operation and the interventional catheter fenestration was I5.5 days (0 - 4448 days). Fourteen interventions (63,6\%) were performed under analog-sedation. After crossing the conduit or patch by stiff wire or Brockenbrough technique a stent was placed in the fenestration after gradual balloon dilatation in all patients. The mean arterial oxygen saturation decreased from $92.5 \%$ ( $=22$; SD $5.5 \%)$ before to $84.2 \%(n=21$; SD $5.5 \%)$ after intervention. Serious complications, especially bleedings were not observed. After a median period of 16 months (0 - 69 months), the fenestration could be shown open by color Doppler echocardiography in 13 patients (68,4\%). In 6 patients (3I,6\%) the fenestration had already closed spontaneously.

Conclusion: Based on this case series, we showed that the catheter interventional reopening of a closed fenestration or creation of an entirely new fenestration in children with TCPC is a therapeutic option in case of failure of the Fontan circulation. It is a low risk procedure even in TCPC with an extra-cardiac conduit. Therefore, this procedure has a big advantage over surgery, which in a similar situation would be risky and complex.

\section{Abstract no: 150}

\section{Oval fossa defects: Morphologic variations and impact on trans-catheter closure}

\section{Joseph Vettukattil", Zaheer Ahmed*, Anthony Salmon*, Timothy Mohun ${ }^{\#}$ and Robert Anderson ${ }^{\dagger}$}

"University Hospital Southampton NHS Trust, Hampshire, United Kingdom

\#National Institute for Medical Research, Medical Research Council, London, United Kingdom

tInstitute of Genetic Medicine, Newcastle University, Newcastle-on-Tyne, United Kingdom

Background: Incomplete formation of the partition between the 2 atrial chambers in the region of the oval fossa results in a range of defects, which extend from PFOs to large secundum ASDs. There is wide variation in the morphology of the latter lesions. The spatial orientation of the margins of ASDs relative to the persisting flap valve is not easily definable with standard echocardiographic imaging. Careful evaluation of the morphology is essential in optimising successful transcatheter closure to minimise complications. The advent of three-dimensional transoesophageal echocardiography (3-DTOE) has changed our understanding of the morphology of these defects, and facilitates successful percutaneous closure.

Methods and results: Since 2007, over a 4 year period, we performed transcatheter closure of ASDs in 104 patients. During this period there were 2 instances of embolisation of the device. We carefully evaluated the morphology of the defects in the patients suffering embolisation, and noted an unusual spiral configuration of the flap valve relative to the rims of the oval fossa. These findings were then found in 4 additional patients, and serve as the focus of this report. To facilitate our understanding of the unusual morphology, we compared the clinical findings with images showing the mechanism of development of the atrial septum in the mouse, revealing a striking similarity.

Conclusions: Though uncommon, spiral spatial orientations of the margins of ASDs are predisposed to embolisation of devices used in percutaneous closure. Standard cross-sectional techniques have limited use in identifying this variation. Understanding the development of the atrial septum in the mouse heart may help explain morphogenesis of the defect, and the mechanism pre-disposing to embolisation. 


\section{Abstract no: 190 \\ Premature closure/restriction of the foramen ovale with severe pre-natal cardiac asymmetry: Will the left heart be sufficient post-natally?}

Karlien Carbonez*, Francoise Mascart" and Catherine Barrea*

"Saint-Luc University Clinics, Brussels, Belgium

\#Saint-Vincent (CHC) Clinic, Rocourt, Belgium

Background: Premature closure of the foramen ovale (FO) is a rare, but serious condition that can be associated with severe right heart failure and hydrops, supraventricular tachycardia and left heart obstructive lesions.

Methods: We report 2 cases of early pre-natal restriction/closure of the FO. Both patients were referred for fetal echocardiography, at $22+4$ and $26+I$ weeks respectively, for severe cardiac asymmetry with suspicion of "hypoplastic left heart" (HLH) at US.

Results: In the Ist case, a 30-year-old GIPO woman, cardiac asymmetry was initially attributed to an aortic coarctation (CoAo). Flow in the FO appeared normal up to $29+3$ weeks. At 34+3 weeks, cardiac asymmetry worsened with a bulging septum towards the left atrium. At 37+I weeks, the left heart appeared tiny with no visible inter-atrial flow. A $2.3 \mathrm{~kg}$ baby-boy was born at $37+3$ weeks. Post-natal echocardiogram showed a tiny restrictive FO, a dilated right heart with transient pulmonary hypertension and a smallish left heart. The aortic arch was small and disharmonious but no CoAo developed. In the 2nd case, a 32-year-old G2PO patient, cardiac asymmetry was immediately attributed to a restrictive FO. A CoAo was suspected at $31+1$ weeks and the FO was closed at $34+4$ weeks with increasing cardiac asymmetry. $2.5 \mathrm{~kg}$ baby girl was born at $38+$ I weeks. Post-natal echocardiography confirmed an intact atrial septum with suspicion of $\mathrm{HLH}$, a bicuspid aortic valve and CoAo. She was treated with prostaglandins. The follow-up echocardiograms showed a progressively better-filled left heart and reduction of pulmonary pressures. She underwent a CoAo repair at day 13. Both children are currently well at respectively 6-year and 2-month follow-up.

Conclusions: Premature closure of the FO may mimic HLH pre- and post-natally. In some cases, despite severe cardiac asymmetry, post-natal evolution may be very good. Further research is necessary to better predict outcome.

\section{Abstract no: 196}

Percutaneous tricuspid valve-in-valve implantation: A paediatric case report

Baher Hanna*, Josep Rodés-Cabau', Suzanne Vobecky", Marie-Josée Raboisson* and Nagib Dahdah*

"Division of Paediatric Cardiology, Ste-Justine Hospital, Montreal, Quebec, Canada

\#Division of Cardiothoracic Surgery, CHU Ste-Justine, University of Montreal, Canada

tDepartment of Cardiology, Quebec Heart \& Lung Institute, Laval University, Quebec, Canada

Background: Tricuspid valve disease proposes a challenge to surgical treatment because of high morbidity and mortality rates. Advances in interventional cardiology introduced the use of bioprosthetic valved stents for the replacement of dysfunctional valves, initially the pulmonary valves and then the aortic valves. Few reports followed for the use in the atrio-ventricular positions.

Materials and methods: A I 4 year-old adolescent with Ebstein's anomaly received a Medtronic MOSAIC bioprothesis replacement of the tricuspid valve at the age of 6 years. Seven years later progressive symptoms related to right-sided heart failure due to severe bioprosthetic valve immobility causing severe regurgitation and moderate stenosis required reintervention. The procedure was performed under general anaesthesia and 3-D TEE guidance. A femoral approach was utilised, the dysfunctional valve crossed by a balloon floatation catheter, a super-stiff guide wire was placed in the left pulmonary artery, over which a 24 Fr RetroFlex 3 system was advanced and flexed at the right atrium but failed to cross the bioprothesis at this point. A pre-dilatation through another femoral access was performed and enabled the SAPIEN system to cross the valve. An inverted Edwards SAPIEN $23 \mathrm{~mm}$ valve was deployed with slow inflation technique under controlled apnea and rapid ventricular pacing. A stable position was assured by a small waist on fluoroscopy. The right atrial pressure rapidly dropped to $7 \mathrm{mmH} g$ with mild central regurgitation on TEE. The patient was extubated after a few hours. The following day fluoroscopy and echocardiography confirmed a stable position with a peak RA-RV gradient of $8 \mathrm{mmHg}$ and a mean of $4 \mathrm{mmHg}$ with mild central regurgitation. The patient was discharged from the hospital after 3 days on daily aminosalicylic acid.

Conclusion: We report a successful percutaneous tricuspid valve replacement for a dysfunctional bioprosthetic valve. Future developments of new material specifically adapted to the tricuspid position is likely to broaden the applicability of this relatively minimally invasive technique.

\section{Abstract no: 224 \\ Transcatheter closure of atrial septal defect closure in small children and infants}

\section{Edwin Francis, Shine Kumar and Raman Krishnakumar}

Amrita Institute of Medical Sciences, Cochin, India

Introduction: Transcatheter closure of atrial septal defect (ASD) is the procedure of choice for central fossa-ovalis defects. However, there is very limited information on safety and follow-up of transcatheter closure of ASD in young children.

Objective: To describe our institutional experience on transcatheter closure of atrial septal defect in infants and children weighing $\leq 10 \mathrm{~kg}$.

Methods: Records of 56 patients weighing $\leq 10 \mathrm{~kg}$ (mean age: $28.70 \pm 9.54$ months, range 4 - 50 months; mean weight: $8.98 \pm 1.29 \mathrm{~kg}$, range 4 - I0kg) who underwent transcatheter closure of ASD in our Institute (January 2007 - December 20I I) were reviewed.

Results: The study population represented $8.6 \%$ of our total experience in this period. Indications for closure included failure to thrive (75.I\%), recurrent respiratory infections (39.4\%) and or heart failure (7.2\%). Case selection was through trans-thoracic echocardiography. The mean ASD size was $14.66 \pm 3.27 \mathrm{~mm}$ (range $9-23 \mathrm{~mm}$ ) and mean pulmonary artery pressure was $27 \pm 6.42 \mathrm{~mm}$ of $\mathrm{Hg}$. Closure was achieved in all with mean fluoroscopy time of $8.6 \mathrm{I} \pm 6.75 \mathrm{minutes}$; device size ranged from $9-24 \mathrm{~mm}$. General anesthesia and transesophageal echo guidance was utilized in 22 (39.3\%). One patient had embolisation of device immediately after release and was surgically retrieved. Transient ECG abnormalities included first degree atrioventricular (AV) block (2) and junctional rhythm (3). All had normal sinus rhythm at 24 hours after procedure. On follow up (median 6 months; range I - 53 months) all were symptom free with over all improved weight $z$ scores and no instances of impingement on adjacent structures.

Conclusion: The immediate and short-term follow-up results of transcatheter device closure of ASD are encouraging and suggest that the indications can be broadened to include selected children $\leq 10 \mathrm{~kg}$. 


\author{
Abstract no: 226 \\ Andrastent in the management of coarctation of the aorta \\ Jacek Bialkowski, Malgorzata Szkutnik and Roland Fiszer \\ Medical University of Silesia, SCCS Zabrze, Poland
}

Background: Stenting in coarctation of the aorta (CoAo) has emerged as an alternative to surgery with a good intermediate result. Recently a new bare metal stent made of a cobalt-chromium alloy (namely Andrastent XL, XXL, Andramed, Germany) was introduced to clinical practice. Its strong radial force, flexibility and good radio-opacity should be advantageous in implantation in CoA.

Objective: To evaluate the use of Andrastent $\mathrm{XL}$ and $\mathrm{XXL}$ in the management of CoA at a single tertiary care centre with regards to immediate results and mid-term follow-up.

Methods: Andrastents were implanted over a 30-month-period in 29 patients: 25 with native CoA and 4 with recurrent after previous surgery (ReCoA). The Andrastents were manually mounted on Maxi LD and BIB balloons and delivered through 10 to 14 Fr Mullins sheaths using a conventional femoral approach. Results: Mean patient age was 28.3 15.6 (from 9 - 65) years. The systolic gradient across the native CoA decreased from a mean $48.3 \pm 20.2$ before to $11.9 \pm 10.2 \mathrm{mmHg}$ after the procedure and in case of ReCoA from $37.8 \pm 20.7$ before to a mean $9.7 \pm 12.4 \mathrm{mmHg}$ after the procedure. No aneurysm formation, stent migration, or rupture of the aorta was observed in any patient during the procedure. The mean fluoroscopy time was $6.1 \pm 2.3$ minutes. Procedural outcome remained favourable during mean follow-up I.I \pm 0.8 without stent fracture. Planned re-dilatation of implanted stent was performed between 4 and 14 months in 6 patients. In one man with secondary LV failure with EF I5\% (49 years old) the procedure was performed urgently during cardiogenic shock with good clinical result.

Conclusions: Implantation of Andrastents $X L$ and $X X L$ is a very good therapeutical option for the treatment of native and recurrent CoA.

\title{
Abstract no: 235 \\ Pre-natal diagnosis of heterotaxy syndrome: Impact of subtype on peri-natal and short term outcome
}

Maria Clara Escobar", Yishay Salem", Brian Kalish", Rahul H. Rathod", Tal Geva", David W. Brown", Sitaram Emani" and Wayne Tworetzky"

"Boston Children's Hospital, Massachusetts, United States of America

\#Harvard Medical School, Boston, Massachusetts, United States of America

Objective: To assess the influence of heterotaxy syndrome (HS) subtype after pre-natal diagnosis (Dx) on peri-natal and short-term survival.

Methods: We included fetuses with HS from 1995-20II. We collected data on gestational age (GA) at Dx, cardiac anomaly, congenital heart block (CHB), extra cardiac anomalies, pregnancy outcome, surgery, current survival and circulation.

Results: Of the 154 fetuses, 6 I (40\%) were categorised as asplenia syndrome patients (ASP) and 93 (60\%) polysplenia syndrome patients (PSP). Median GA at Dx was 21 (range 14 - 39 weeks). Complex cardiac anomalies were more frequent in ASP than PSP (98\% vs. 58\%). Bradycardia-CHB was exclusive to PSP (18/93). Extra cardiac anomalies were present in 45\%. In ASP, 24/36 (66\%) Dx<24w elected for termination of pregnancy (TOP); 3 fetuses died from noncardiac lesions; and 34 (56\%) were live born. In PSP, I I/6I ( I 8\%) Dx<24w elected for TOP: 5 fetuses died (4 with CHB); and 77 (83\%) were live born. Mean follow-up was 6.1 years $( \pm 4.6)$. In the live born ASP, I5/34 (44\%) died: 6 with pulmonary vein stenosis; 5 post-operatively: and 4 from non-cardiac lesions. In the live born PSP 10/77(13\%) died: 6 postoperatively; 2 with CHB; and 2 from extra cardiac lesions. Of the current survivors I00\% of ASP underwent cardiac surgery compared to $53 \%$ of PSP. Starting with fetal Dx, 64/93 (69\%) of PSP are still alive, 57\% with biventricular circulation. Of the ASP, I $7 / 6$ I (28\%) are still alive only $5 \%$ biventricular.

Conclusion: After fetal Dx there were significant peri-natal survival differences between ASP and PSP. ASP had higher rates of TOP and post-natal mortality. The majority PSP were biventricular compared to rather few ASPs. CHB was a risk factor for fetal demise. Neonatal deaths were cardiac related in both groups whereas late deaths were due to extra cardiac causes in PSP, and to pulmonary vein stenosis in ASP.

\section{Abstract no: 249}

Ventricular septal defect closures with a new platinum-coated nitinol device

\section{Pornthep Lertsapcharoen*, Suphot Srimahachota*, Phingphol Charoonrut" and Kanyalak Vithessondhi}

"Chulalongkorn University, Pathum Wan, Bangkok, Thailand

\#Mahidol University, Phutthamonton, Nakhon Pathom, Thailand

Background: New platinum-coated nitinol VSD (CocoonTM) devices were designed into 3 types: perimembranous, aneurysmal peri-membranous and muscular VSD devices according to the morphology of the VSDs. This study reported the animal trial and early clinical results.

Materials and methods: In the animal trial, VSD was created in 12 pigs by retrograde aortic approach for ventricular septal puncture with Brokenborough needle and followed by balloon dilation of the septum. The clinical trial included 16 VSD patients. Age ranged from 2 - 56 years, weight ranged from 10 - $72 \mathrm{~kg}$. There were 8 peri-membranous, 5 aneurysmal, 2 muscular and I post-myocardial infarction VSDs.

Results: In the animal study, the device was successfully deployed to close the created VSD in the I 2 pigs. Imaging studies demonstrated complete VSD closure in II animals. The autopsy findings demonstrated minimal and complete neo-endothelialisation over the device at I week and 8 weeks after implantation, respectively. In the clinical trial, the device was successfully deployed in 14 patients: 2 unsuccessful cases underwent surgical closure; $2 / 14$ successfully deployed cases developed haemolysis after the procedure; I due to residual VSD leakage; and another one due to undiagnosed aortic regurgitation with the regurgitant flow hitting on the device. Both of them had surgical device removal and VSD closure. On echocardiographic follow-up over a period ranged from I to 6 months, 3 cases still had tiny leakage. There was no device-related complication.

Conclusion: The new nanoplatinum-coated nitinol VSD devices had a good outcome in animal trials. It also had an attractive result in early clinical trials. 


\section{Abstract no: 277 \\ Device closure of very large atrial septal defects}

Edwin Francis", Artur Lopes" and Raman Krishnakumar*

"Amrita Institute of Medical Sciences, Cochin, India

\#Hospital, Lisbon, Portugal

Background: Device closure of small to moderate sized atrial septal defects (ASD) are well accepted. But device closure of very large defects using 40mm or larger devices are technically challenging and there limited data on such procedures. We retrospectively analysed large devices used in our institute.

Methods: We retrospectively analysed large devices used in our institute. From January 2006 - June 20I2, all ASD device closure attempts using devices 40mm or above were analysed.

Results: 18 patients had an ASD device closure attempt using devices $40 \mathrm{~mm}$ in size or larger. Mean age of patients were $35 \pm 13$ years; I I of them were male. ASD measured mean of $38 \mathrm{~mm} \pm 2 \mathrm{~mm}$. Fluorotime for the proceudre mean was 13.9 minutes \pm 10.2 minutes (median was I0.0 minutes). Device size was $40 \mathrm{~mm}$ in 12pts; $42 \mathrm{~mm}$ in 2 pts; $46 \mathrm{~mm}$ in 2pts. The procedure was successfull in 14 pts. Three patients had immediate device embolization (device size $40 \mathrm{~mm}$ ) requiring emergency surgery. One patient had the procedure aborted as the $46 \mathrm{~mm}$ device could not effectively catch the post-inferior margin and was electively sent for surgery. One patient had acute pulmonary edema in the immediate post-procedure period. There were no hospital deaths or other major complications. Mean follow-up time was 10 months \pm 9 months (median 3 months). No major cardiovascular events were noticed on follow-up. Two patients were on atrial fibrillation, both of them with previous history of persistent or permanent AF.

Conclusions: Very large defects can be safely considered for trancatheter closure, with a higher chance of failure or embolisation rates.

\section{Abstract no: 287 \\ Outcome of transcatheter device closures of peri-membranous ventricular septal defect}

Fang Liu, Wu Lin, Feng Sheng, Lan He, Chunhua Qi, Haiyan Deng and Lifeng Zhang

Heart Centre, Children's Hospital of Fudan University, Shanghai, China

Purpose: To evaluate the safety and efficacy of transcatheter closure for perimembranous ventricular septal defect (PmVSD).

Methods: From September 2002 - June 20I I, I55 PmVSD patients were admitted for percutaneous device closure; aged from 26 months - 21 years and weight from 1 I - 67kg. Among these 155 patients, 12 patients were post-surgery residual VSD, I patient was associated with Down's syndrome. All patients had transthoracic echocardiography (TTE), ECG and 24h Holter pre- and post-occlusion, and were asked to follow-up in Im, 3m, $6 \mathrm{~m}$, $12 \mathrm{~m}$; then once every year for 5 years; and then every 3 years. ECG and TTE were performed in every follow-up, and 24h Holter was performed only when necessary.

Results: Devices were deployed successfully in 151 patients (97.5\%). The VSD size measured by left ventricle angiogram was $2.0-8.46 \mathrm{~mm}$ on right ventricle side with multiple shunt in most patients with aneurism, and Qp/Qs was 1.4-2.18. The device used included 4 types with varying shape of the left disc and the height, and size ranged from $4 \mathrm{~mm}-12 \mathrm{~mm}$. All patients were followed up until June 2012, with the follow-up duration 12 - $82 \mathrm{~m}$. During follow-up, 15 immediate adverse events (9.9\%) were reported, including 2 complete AVB, 5 CRBBB, 3 CLBBB, I premature ventricular contraction, and 4 moderate TR. Almost all patients recovered, except 3 CRBB and 2 moderate TR which still presented with normal heart chamber and function. There was also I late complete AVB in 3-month follow-up. No patient required a permanent pacemaker or surgery till now.

Conclusions: Transcatheter PmVSD closure can be performed safely and successfully with low morbidity and mortality. Medium to long term prognostic results are favourable, however, late complications especially high-degree AVB should also be considered by all intervention cardiologists.

\section{Abstract no: 317 fetal heart rate monitor \\ Bettina Cuneo*, Farhan Hanif*, Carolyn Sullivan*, and D. Woodrow Benson ${ }^{\dagger}$ \\ "Advocate Hope Children's Hospital Chicago, Illinois, United States of America \\ \#Advocate Lutheran General Hospital, Chicago, Illinois, United States of America \\ †Children's Hospital of Wisconsin, Milwaukee, Wisconsin, United States of America}

Heart sounds at home: Prospective maternal surveillance of SSA positive pregnancies using a hand-held

Background: The purpose of this study was to test a simple hand-held Doppler fetal heart rate (FHR) monitor in the ambulatory setting to prospectively identify the onset of AV block. This may be useful in monitoring maternal SSA/SSB disease.

Methods: We recruited pregnant women with SSA and/or SSB antibodies at gestational age (GA) 16 - 17 weeks from a large peri-natal cardiology practice. Mothers were instructed and then given hand-held Doppler FHR monitors. Between 17 - 27 weeks, mothers were instructed to listen daily for I minute twice daily and seek attention immediately if there were "skipped beats" or a "slow rate" (<120bpm). The mothers returned to clinic every other week until GA 27 weeks for a fetal echocardiogram to assess the mechanical PR interval, endocardial fibro-elastosis (EFE) and AV valve insufficiency. At the conclusion of the monitoring period the mothers filled out a questionnaire rating their experience. Newborns were evaluated by I2-lead ECG to confirm verify conduction.

Results: Between 2009 and 2012, II mothers with SSA (9) or SSA/SSB (2) antibodies were given monitors at GA I6.5 - 18 weeks. 3 mothers previously had a child with AV block. Fetal AV block recurred in 2 of 3 fetal siblings. Each fetus had "skipped beats" at GA 17.5 and 18 weeks, 12 hours after auscultation of normal fetal heart tones. The mother of Fetus number I sought medical attention 24 hours later; the fetus had EFE and $3^{\circ} \mathrm{AV}$ block. The mother of Fetus number 2 was seen within 4 hours; her fetus had intermittent $2^{\circ} \mathrm{AV}$ block. Both mothers were given high dose vitamin D, IVIG and dexamethasone. Sinus rhythm was restored in Fetus number 2 and the AV block in Fetus number I unchanged. No fetus with normal FHR Doppler monitoring $(n=9)$ had echocardiographic abnormalities; echo did alter later management in Fetuses number I and number 2. 90\% of mothers were reassured by the FHR monitor, would recommend it and use it during subsequent pregnancies.

Conclusions: This pilot study suggests that by using a simple FHR monitor, mothers can prospectively detect the onset of SSA-mediated conduction system abnormalities. This capability may make early successful treatment feasible. 


\section{Abstract no: 322 \\ Percutaneous balloon angioplasty for severe native aortic coarctation in young infant $<6$ months}

Fang Liu, Wu Lin, Lan He, Chunhua Qi, Haiyan Deng, Lifeng Zhang and Guoying Huang

Heart Centre, Children's Hospital of Fudan University, Shanghai, China

Purpose: To assess the effects and the potential role of percutaneous transcatheter balloon angioplasty as an alternative therapy to surgical operation. Methods: Thirty patients aged 6d - 6m with severe coarctation of the aorta were admitted for balloon angioplasty, with body weight 2.5 - $6.1 \mathrm{~kg}$. All 30 patients had cardiac dysfunction, and 8 patients had cardiac shock with severe metabolic acidosis. Ten patients were isolated CoA, while the others were associated with other cardiac malformations. Cardiac catheterisation and aortic angiogram were performed under general anesthesia with intubation. Balloon size was from $3 \times$ $20 \mathrm{~mm}-8 \times 20 \mathrm{~mm}$, and PTCA balloon was preferred for its low profile and small sheath, and then is Cordis balloon.

Results: Femoral artery was successfully punctured in all patients, except for I failed patient who had an additional carotid artery puncture. The pressure gradient (PG) across the coarctation was $13-76(40 \pm 17.0) \mathrm{mmHg}$. The narrowest diameter was $0.5-2.8$ ( $1.7 \pm 0.6) \mathrm{mm}$. All patients had successful dilatation with PG significantly decreasing to 0 - 40 ( I $4.7 \pm$ I I.7) $\mathrm{mmHg}$ and the diameter improved significantly to $2.5-4.8 \mathrm{~mm}(3.7+0.9)$. There were no intra-operative complications in all patients. However, in the case with the carotid arteries puncture, a giant aneurysm formed in the puncture site and required surgical repair. During 6 months - 5 years follow-up, it was shown that: (I) pressure gradient crossing coarctation measured by echocardiography further decreased or remained stable in 28 cases. The remaining 2 patients, whose pressure gradient gradually increased, needed a second dilatation. No case needed further surgery because of CoA; (2) Aortic aneurysm formation in I case, which disappeared in I8m; (3) Tricuspid regurgitation and pulmonary hypertension improved in all patients; (4) all patients were asymptomatic.

Conclusions: Percutaneous balloon angioplasty is relatively safe and effective and should be considered as a valid alternative to surgical operations considering its good effect, less trauma and low complications in patients.

\section{Abstract no: 343}

Outcome and feasibility of transcatheter atrial septal defect occlusion under transthoracic echocardiography guidance in resource-limited centre: Initial report

\section{Rahmat Budi Kuswiyanto and Sri Endah Rahayuningsih}

Department of Paediatric and Child Health, Faculty of Medicine, Padjadjaran University, Hasan Sadikin Hospital, Bandung, Indonesia

Background: Transcatheter ASD occlusion has become the established treatment of secundum ASD under transoesophageal echocardiography (TOE) guidance in many centres, nevertheless TOE may not available in several centres.

Aim: To evaluate the outcome and feasibility of transcatheter ASD occlusion under transthoracic echocardiography (TTE) guidance in our institution.

Methods: TTE was performed to assess the suitability of transcatheter occlusion. The occlusion procedures were performed under sedation and local anaesthesia. Routine right heart catheterisation was performed in all cases to assess the pulmonary vascular resistance. Balloon sizing was done in case of inadequacy of the rims. Amplatzer Septal Occluder device was used for occlusion. TTE and angiogram were done to assess the position of the device and the result of occlusion. All patients were followed-up periodically by clinical, ECG and TTE at 24 hours and I, 6 and I 2 months after closured.

Results: Out of a total of 45 patients who underwent non-surgical cardiac interventions from April 201। - December 201।, there were 9 cases of secundum ASD which were suitable for occlusion. The median of age, weight, and diameter of defect was 15 years (ranged from 10 - 42 years); $32 \mathrm{~kg}$ ( 27 - $54 \mathrm{~kg}$ ); and $24 \mathrm{~mm}(16-34 \mathrm{~mm})$, respectively. 7 patients had achieved complete immediate closure, and 2 cases failed due to limitations in assessing the inferior and floppy of posterior rim. There were no complication during procedures and followed-up; all patients were discharged the next day; and complete closure was seen on further followed-up.

Conclusions: Transcatheter ASD occlusion may perform under TTE guidance which showed effectiveness and safe therapy, but it has limita-tions in assessing the inferior and floppy posterior rim. Further evaluation of this method is mandatory.

\section{Abstract no: 349}

\section{Closure of ventricular septal defects by Lê Pfm coil}

\section{Keyhan Zanjani", Aliakbar Zeinaloo* and Trong Phi Le"}

"Tehran University of Medical Sciences, Tehran, Iran

\#University of Hamburg, Hamburg, Germany

Background: Transcatheter closure of ventricular septal defects (VSD) is a promising procedure. Although it is limited to smaller VSDs, its apparent advantages over surgical closure (lesser traumatic burden, better esthetic result, and avoidance of cardiopulmonary bypass) made it the preferable method of VSD closure whenever applicable. In the near past, Amplatzer occluders were the leading devices for this purpose. However, high risk of heart block is a great concern. Lê Pfm coil is a newer device, which causes less heart block due to its softer consistency.

Methods: Since March 2009, we attempted to occlude 9 VSDs using Lê Pfm coil. The cohort consisted of 6 female and 3 male patients with a median age of 6 years (4 - 9). Indications for VSD closure were Qp/Qs>1.5 in 7 and/or left ventricular hypertrophy in 5 patients. The smallest VSD diameter on echocardiography was $3.7 \pm 0.7 \mathrm{~mm}$. Mean pulmonary artery pressure was $32 \pm 14 \mathrm{mmHg}$. One patient had a patent ductus arteriosus (PDA) closed by pfm coil previously, while PDA of another patient was closed at the same time by a similar device.

Results: We have successfully implanted two $8 \times 6$, four $10 \times 6$, and $12 \times 6,14 \times 8$, and $16 \times 8$ coils one each. VSDs were occluded in $2 / 9$ immediately or on the next day, 4/7 after 6 months, and 4/5 after I year. One patient (I6 8 coil) developed trivial Al (improved during the follow up) and transient complete heart block (I day). No other major complication occurred.

Conclusion: Lê Pfm coil is an attractive device for VSD closure. Late closure was the main drawback of this device in our cohort. 


\section{Abstract no: 356 \\ Pre-natal fetal echocardiographic findings in total anomalous pulmonary venous return in 25 patients}

Suguna Ganesan, Michael Brook, Norman Silverman and Anita Moon-Grady

University of California, Oakland, California, United States of America

Background/hypothesis: Pre-natal diagnosis of total anomalous pulmonary venous return (TAPVR) is crucial as there is the clinical possibility that it could obstruct venous return - a condition which only manifests post-natally. Optimal peri-natal management in these cases involves timely identification and delivery at an appropriate centre. Ante-natal diagnosis has been considered a challenge.

Aim: To show that consistent pre-natal echocardiographic features exist in TAPVR.

Methods: This was a retrospective cohort review of fetal echocardiographic studies performed at the University of California, San Francisco from 2001 - 2012. We systematically reviewed the sonographic features that led to the diagnosis of TAPVR in each case.

Results: Over the study period, 25 patients were diagnosed pre-natally with TAPVR, at a mean gestational age of 24 weeks: 4 were isolated cases of abnormal pulmonary venous return; 21 had heterotaxy syndrome with additional cardiac abnormalities. Abnormal connections were supracardiac in 18 cases, cardiac in I and infracardiac in 6 . Identification of a venous confluence posterior to the left atrium on axial images and presence of additional vertical venous channels apart from the vena cavae on the 3-vessel view or sagittal views were consistent diagnostic markers on 2-D imaging. Cardiac asymmetry was not consistently noted. The most consistent Doppler finding was one of low-velocity mildly pulsatile mono- or biphasic flow in individual pulmonary veins, seen in $24 / 25$ (96\%) of the patients studied, irrespective of presence or absence of downstream obstruction.

Conclusions: The diagnosis of TAPVR can be suspected by a targeted approach with attention to important B-mode and spectral Doppler clues. Diagnosis can be suspected from standard cardiac views used in routine anomaly screening. The spectral Doppler waveform pattern is extremely helpful in identifying patients with abnormal connections and in patients with isolated TAPVR it may be the most useful finding upon screening when gross 2-D and colour Doppler appearance is apparently normal.

\section{Abstract no: 358 \\ Left heart structures in fetuses with congenital diaphragmatic hernia and the effect of fetal endoscopic tracheal occlusion}

Luciane Rocha", Francesca Byrne", Roberta Keller", Doug Miniati", Michael Brook\#, Norman Silverman" and Anita Moon-Grady"

"University Federal of Sao Paulo, São Paulo, Brazil

\#University of California, Oakland, California, United States of America

Background/hypothesis: Small left heart structures have been observed in fetuses with left-sided congenital diaphragmatic hernia (CDH). Compression from herniated abdominal organs, and reduced filling of the adjacent left ventricle, seem to be the main pathophysiologic mechanisms. Foetoscopic tracheal occlusion (FETO) is a novel procedure performed in mid-gestation which seems to promote lung growth in fetuses with CDH; however, the cardiac effects of FETO are poorly described.

Aim: To study the effects of FETO on the heart and analyse the left cardiac structures size at birth.

Methods: This is a retrospective case-control study. We performed measurements of mitral, tricuspid, aortic and pulmonary valve and pulmonary artery diameters, ventricular lengths, left ventricular end-diastolic volume indexed (LVEDVi) to body surface area. Comparisons were made between fetuses that underwent FETO and matched controls with similar $\mathrm{CDH}$ disease severity and fetal cardiac dimensions who did not undergo fetal intervention.

Results: A total of 35 fetuses were studied: 9 had FETO and 26 were controls. All had liver herniation and lung-head ratio $<$ I at fetal evaluation (average GA 24 weeks). At birth, prior to CDH repair, the intervention group had: larger LVEDVi $(16.8 \mathrm{ml} / \mathrm{m} 2 \mathrm{vs} .12 .76 \mathrm{ml} / \mathrm{m} 2, \mathrm{p}=0.04)$; increased $\mathrm{LV}$ length $z$-score $(-2.05 \mathrm{vs}$. $-4, p=0.006)$; larger $L V: R V$ length ratio (I.43 vs. I.04, $p=0.03)$; increased LPA diameter $z$-score $(1.71$ vs. $-1.04, p=0.021)$; and better growth of aortic valve $(-1.66$ fetal to -2.18 neonatal in FETO, -1.09 fetal to -3.3 neonatal in controls, $p<0.005)$. A trend towards better growth of the mitral and tricuspid valves was observed. Conclusions: Left heart structures and LPA were larger post-natally in fetuses with CDH who underwent FETO than in those who did not. These data suggest that haemodynamic alterations are introduced with fetal tracheal occlusion which are associated with alterations in ventricular loading and may influence growth; further study of these observations is necessary to determine overall significance.

\section{Abstract no: 365 \\ The relationship between left heart hypoplasia and congenital diaphragmatic hernia in human fetuses \\ Francesca Byrne, Roberta Keller, Jeffery Meadows, Doug Miniati, Michael Brook, Norman Silverman and Anita Moon-Grady \\ University of California, San Francisco, California, United States of America}

Background: Congenital diaphragmatic hernia $(\mathrm{CDH})$ is associated with small left ventricle (LV), mitral valve (MV) and aortic valve (AV) in utero, but the pathophysiologic processes behind this observation and implications for prognosis are not well known.

Hypotheses: We hypothesised that both left- and right-sided CDH severity would correlate with worsening left heart hypoplasia and lower left cardiac output. We analysed factors leading to abnormal fetal blood flow patterns that potentially contribute to left heart hypoplasia: compression, altered ductus venosus and reduced pulmonary blood flow.

Materials and methods: Retrospective cohort of fetuses with CDH from 2000 - 20 I 0. Ultrasound-derived lung:head ratio (LHR), liver position and hernia side were recorded. $C D H$ severity was categorised as severe ( $L H R<I=1.0$ and liver herniated into thorax) or mild (LHR>I.0 or no liver herniation). Cardiac dimensions and combined ventricular output (CVO) were measured on echocardiogram at presentation. Fetuses with anomalies other than $\mathrm{CDH}$ were excluded. I7I left $\mathrm{CDH}$, and 17 right $\mathrm{CDH}$ were included.

Results: Fetuses with severe left CDH had smaller MV ( $Z=-2.24 \pm 1.3$ vs. $-1.33 \pm 1.08)$; $A V(Z=-1.39 \pm 1.21$ vs. $-0.51 \pm 1.05)$ and LV length ( $Z=-1.66 \pm 1.28$ vs. $-1.1 \mathrm{I} \pm 1.45) Z$-scores; and a lower mean $L V$ output as percentage of CVO $(26 \% \pm 10 \%$ vs. $32 \% \pm 10 \%)$; versus those with mild $C D H$ (all $p<0.01)$. Although

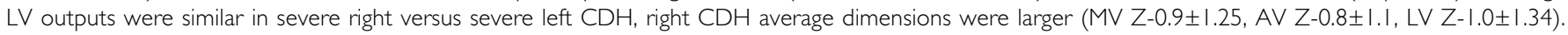
Severe dextroposition and abnormal liver/ductus venosus position were independently associated with smaller left heart structures, while LHR and branch pulmonary artery measures were not.

Conclusions: Severity of left heart hypoplasia correlates with severity of $\mathrm{CDH}$ when the left diaphragm is affected, but not when the lesion is right-sided. Results suggest that both compression by left $\mathrm{CDH}$ and preload alterations from changes in ductus venosus flow are present and may be responsible for poor ventricular growth in $\mathrm{LCDH}$. 


\section{Abstract no: 389 \\ Use of local anaesthetic $(0.25 \%$ bupivacaine) for pain control in paediatric cardiac catheterisation: A randomised controlled trial}

\section{Jacqueline Viegas, Amy Palma, Lee Benson, Cedric Manlhiot and Brian McCrindle}

Labatt Family Heart Centre, The Hospital for Sick Children, Toronto, Canada

Background: In paediatric cardiac catheterisation procedures performed under general anaesthesia, local anaesthetic is used for some patients prior to femoral sheath removal. We found no published reports of the impact of local anaesthetic on pain after paediatric cardiac catheterisation, and mixed reports on its effectiveness in adults.

Aim: Our purpose was to investigate the effects of local infiltration of $0.25 \%$ bupivacaine on self report measures of pain and analgesic use up to 6 hours postprocedure in paediatric patients undergoing cardiac catheterisation procedures.

Hypothesis: We hypothesised that administering $0.25 \%$ bupivacaine around the femoral catheter insertion site in children under general anaesthetic at the end of cardiac catheterisation procedures would have no effect on pain scores and use of analgesics up to 6 hours post-procedure.

Materials and methods: A randomised controlled trial with 140 participants aged 7 - 18 years undergoing cardiac catheterisation under general anaesthesia via femoral vein/artery is being conducted. Participants were randomised to the intervention group receiving usual care plus subcutaneous infiltration of $0.25 \%$ at the femoral site just prior to sheath removal or to the control group who had femoral sheaths removed under general anaesthetic but without subcutaneous bupivacaine.

Results: To date 133//40 participants have been recruited and followed; the remaining 7 will be recruited by September 20 I 2. Blinded mid-point data analysis was conducted for data safety monitoring purposes and found that recruitment should continue. Full results will be available by October 2012 . Primary outcomes are patient-rated pain scores and use of analgesics post-operatively. Procedural information, such as size and number of catheters used and post-operative bleeding, will also be compared between groups.

Conclusion: This study's results will be the first to contribute to an evidence base regarding the effectiveness of $0.25 \%$ bupivacaine given just prior to femoral sheath removal for pain control after paediatric cardiac catheterisation

\section{Abstract no: 391 \\ Coronary interventions in children with congenital heart diseases}

\section{Peter Zartner and Martin Schneider}

Cardiology, German Paediatric Heart Centre, Sankt Augustin, Germany

Background: The small vascular anatomy of infants and children makes interventional treatment of impaired coronary perfusion, such as stenosis, complete occlusions and fistulae demanding. Appropriate materials and techniques for this young age group, have to demonstrate their ability to effectively treat these lesions and avoid problems, such as disruption, myocardial infarction or malfunction.

Methods and results: Between 2004 and 20I I, I 4 patients aged 9 days - 25 years (median 4.6 years) with a bodyweight of I.7 - $65 \mathrm{~kg}$ (median I 4kg) underwent coronary intervention. In 3 cases emergency revascularisation of the left coronary artery was performed successfully, followed by stent implantation in I patient. Embolisation of the coronary arterial fistulae with coils and vascular plugs was effective in 10 patients. An antegrade, retrograde or combined approach to achieve the most distal device placement preserved all side branches. One infant with pulmonary atresia and an intact ventricular septum was prepared for biventricular repair by step-by-step closure of the right ventricular to the coronary artery connections. No procedure-related deaths occurred.

Conclusion: Congenital and post-procedural coronary obstructive lesions can be treated effectively and saved with balloon dilation at any age. In coronaries impaired by external compression, stent implantation can restore perfusion but long term results are still missing. Interventional closure of coronary fistulae improves peripheral coronary arterial perfusion. A combination of these strategies allows the preparation of patients with pulmonary atresia and intact ventricular septum for biventricular repair.

\section{Abstract no: 392}

Interventional closures of muscular VSDs in young patients

\section{Peter Zartner, Nicole Toussaint Goetz, Viktor Hraska and Martin Schneider}

German Paediatric Heart Centre, Sankt Augustin, Germany

Purpose: The presence of muscular ventricular septal defects (mVSD) may pose a high circulatory burden in young patients. Surgical closure is difficult because of the multi-layer structure of these malformations.

Method: We present the course of 13 patients (age 10 days - 7.3 years (median 9 months); bodyweight 2.2 and I $8 \mathrm{~kg}$ (median $8.7 \mathrm{~kg}$ ) undergoing transcatheter closure of such defects. All closures were assisted by transoesophageal echo guidance. For transvascular closure of 5 singular and 8 multiple mVSD 5 Amplatzer PDA II occluder, 6 Amplatzer VSD occluder and 4 Amplatzer vascular plugs IV were used. Follow-up time ranges from 23 days to 2.8 years (mean I.2 years).

Results: Three defects observed, and closed immediately. Nine mVSD showed a residual shunt immediately after the implantation procedure, which further reduced during follow-up in all patients. Two intra-procedural complications occurred. A sudden complete AV-Block led to transcatheter explantation of the device in I patient. The patient with this restrictive cardiomyopathy died during follow-up. In I patient on ECMO a left ventricular perforation with the device already in place had to be over-sewn in an emergency operation.

Conclusion: With some of the newer devices available, which pass through smaller delivery sheaths of 4 - $6 \mathrm{~F}$, interventional closure of mVSD has become a feasible option in the treatment of patents of all ages and a bodyweight from $2.2 \mathrm{~kg}$ on, who present with elevated right ventricular pressures and high shunt volume. The procedure is challenging in newborns and infants and severe complications may occur. 


\section{Abstract no: 409 \\ Catheter interventions in congenital heart disease without a regular catheterisation laboratory equipment: The chain of hope experience in Rwanda}

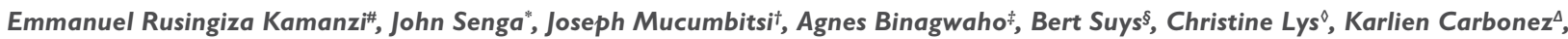

Caroline Ovaert ${ }^{\Delta}$ and Thierry Sluysmans ${ }^{\Delta}$

"Cliniques Universitaires de Kinshasa, Kinshasa, Republique Democratique du Congo

\#Kigali University Teaching Hospital, Kigali, Rwanda

†King Faisal Hospital, Kigali, Rwanda

* Minister of Health of Rwanda

§aediatric cardiology, RUG, Ghent University Hospital, Gent, Belgium

${ }^{\wedge}$ Anesthetist, Belgium

$\triangle$ Paediatric Cardiology, Cliniques Universitaires Saint-LUC, Bruxelles, Belgium

Background: Thousands of children live in developing countries with untreated but correctable congenital heart disease. Most of them will die due to the lack of suitable medical and surgical facilities. Since 2008, visiting teams made by cardiologists and cardiovascular surgeons from Chain of Hope/Belgium make trips in Rwanda to perform cardiac catheterisation and open heart surgery in children presenting congenital heart defects. The aim of this study is to demonstrate the feasibility and the effectiveness of cardiac catheterisation in Rwanda without the support of a sophisticated catheterisation laboratory.

Objectives: We report the feasibility and safety of cardiac catheterisation in a developing country without access to a regular cardiac catheterisation laboratory. Methods: The equipment used for imaging consisted of a mono-plan conventional C-Arm. X-Ray system and a portable ultrasound machine, using usual guidewires and catheters for cardiovascular access.

Results: A total 30 patients, including 17 children under the age of 2 years and 2 adults, underwent catheterisation treatment of their cardiac anomalies: 20 with patent ductus arteriosus, 9 with pulmonary valve stenosis including 2 with critical stenosis, 3 with secundum atrial septal defect. Except for 2 cases requiring surgery, the other patients were successfully treated without complication. They were all discharged from hospital, usually the day after cardiac catheterisation, and showed significant clinical improvement on follow-up.

Conclusion: Cardiac catheterisation can be performed safely and is highly effective in a country with limited resources. If patients are well selected, this mode of treatment is possible without the support of a sophisticated catheterisation laboratory.

\section{Abstract no: 422}

Amplatzer duct occluder II in alien positions

\section{I.B. Vijayalakshmi}

Sri Jayadeva Institute of Cardiovascular Sciences and Research, Bengaluru, Bengalore, India

Background: Amplatzer Duct Occluder II (ADO II) is specially designed for closing long ducts in infants. There are few reports of "off-label" use of ADO II in alien positions.

Aim: To evaluate the advantages and disadvantages of ADO II in alien positions.

Material and results: In this prospective study 5 I cases of VSD and one case of coronary artery venous fistula (CAVF) with I aorto-right ventricular tunnel (ARVT) were all closed with ADO II. 72 cases of VSDs were closed with regular devices. Age ranged from 8 months - 21 years (mean 9.7 years); and male/ female ratio I.I:I: In 2 patients associated ASDs were simultaneously closed with ASD devices; dextrocardia (I); mid or high muscular VSDs (I I); perimembraneous VSDs (38); Gerbode defect (2). In I patient, 2 ADO Ils were used. The shortest fluoroscopic time was 4 minutes, mean was 8.4 \pm 4 .I minutes. Complete closure was achieved in all: I case of Gerbode defect closed with ADO II developed complete heart block and recovered with temporary pacing and steroids while none of the patients developed tricuspid regurgitation or aortic regurgitations. Fluoroscopic time was more (5.6 \pm 3.4 minutes) in the control group: 3 cases had residual shunt and transient complete heart block while haemolysis occurred in I patient each.

Discussions: The advantages of using ADO II are that it has a very low profile and can be easily delivered through a 5F guiding catheter with short fluoroscopic time at a I/3 of the cost of a ventricular septal occluder. The disadvantage is that it cannot be used in VSDs $>6 \mathrm{~mm}$.

Conclusions: ADO II is an excellent device in an alien position like VSD, Gerbode defects, CAVF or ARVT. The procedure time and the cost are significantly less than that of regular devices. The success rate is very high and complication rate is very low.

\section{Abstract no: 425}

Transcatheter closure of atrial septal defects: Single-centre experience in a resource-limited paediatric heart centre

\section{Sukman Putra, Mulyadi Djer, Nikmah Idris, Dedy Wilson and Sudigdo Satroasmoro}

Department of Paediatrics, University of Indonesia, RSCM Jakarta, Indonesia

Background: Transcatheter of atrial septal defects (ASD) has been accepted worldwide as an alternative to surgical closure with good clinical results. This procedure plays an important role in most of the developing countries with limited resources, including: lack of a cardiac surgeon, limited ICU beds, few paediatric cardiac intensivist as well as limited funding.

Objective: To report our clinical experience in transcatheter closure of ASD in a paediatric heart centre with little human resources and very limited funding.

Materials and methods: We successfully performed I 8 transcatheter closure of ASD out of a total of various 556 interventional procedures for congenital and structural heart disease (21.2\%) September 2002 - June 2012. The procedures were performed under general anaesthesia and guided by fluoroscopy and transoesophageal echocardiography.

Results: The median age was 7.6 years (ranged I.3 - 69.3 years) with 93 (78.8\%) female and 25 (21.2\%) male. The majority of patients' body weight was between $10-30 \mathrm{~kg}$. The defect size was $17.8 \mathrm{~mm}$ (ranged $4-36 \mathrm{~mm}$ ) and the devices size implanted was $22 \mathrm{~mm}$ (ranged 10 - 38mm), all using Amplatzer septal occlude (ASO). The mean procedure time was 109 minutes (range 50 - 74 minutes) with the fluoroscopic time was 30 minutes (range 12 - 83 minutes). I patient had an embolised to right ventricle requiring surgical intervention, which means the success rate was $99.1 \%$.

Conclusion: Transcatheter closure of ASD is an effective and safe procedure. In a country with limited resources, this procedure plays an important role in treating atrial septal defects as an alternative to surgery. 
Abstract no: 432

Tailored management approach for critically sick children and late presenters with congenital heart disease

Milad El-Segaier"\# and Mohammed Omar Galal",t

"King Fahad Medical City, PSHC, Riyadh, Saudi Arabia

\#Skåne University Hospital, Lund, Sweden

+University of Essen, Germany

Background and objectives: Re-conditioning before cardiac surgery in critically sick children is often required. We report our experience using tailored management approach in these patients.

Methods and patients: The charts of patients with CHD judged to be a high operative risk were reviewed. Included were patients with: Large left-t- right shunt and ventilation $>2$ months; significant left-to-right shunts at multiple levels combined with malnutrition or recent infection; severely impaired cardiac function requiring inotropic support and anti-failure medications; recent RSV infection; severe malnutrition (body weight < 5th percentile); and critically sick patients during early post-operative course. Excluded were patients with: significant left to right shunts which presented early with minor growth retardation and without recent active infection.

Results: Six patients were included. The median age was 13 months (2 - 48 months) and median weight $4.6 \mathrm{~kg}(2.3-12.6 \mathrm{~kg})$ : 2 patients had multiple left-to-right shunts and ventilator dependency; I with huge VSD presented at 4 years of age; I had low body weight, large VSD and impaired LV function; 2 more had early post-operative complications and ventilator dependency. In the first category, staged approach was essential. This was done by transcatheter closure of the PDA followed by pulmonary artery banding. This approach led to extubation. After proper nutrition, total correction was done. The patient who presented at 4 years of age underwent banding of PA and surgical repair of VSD later. Because of a long post-operative ICU stay, he needed percutaneous closure of his residual VSD and was then discharged. The patient with large muscular VSD and impaired LV function underwent percutaneous VSD closure with good result despite low weight (4.2kg). Of the last 2 patients one needed balloon dilation of the residual coarctation, while the other had stenting of stenoses because of severe obstruction to his bilateral Glenn anastomosis. Both had good results.

Conclusions: In severely sick children and a late presenter with multiple shunts tailored management, including therapeutic catheterisation and supportive measures, are essential before cardiac surgery. In severely sick patients catheterisation and possible intervention should be considered in the early post-operative phase.

\title{
Abstract no: 433
}

Percutaneous closure of coronary artery fistulas: Various embolisation techniques and devices

Ghee Tiong Koh, Sharifah Ainon Ismail Mokthar and Ahmad Rustam Zainudin

Department of Paediatric Cardiology, Penang General Hospital, Malaysia

Background/hypothesis: Percutaneous closure of coronary artery fistulas (CAF) has emerged as an alternative to surgery. Closure of CAF with coils has been well described. We aimed to review our experience about the closure of CAF with various techniques and devices.

Materials and methods: We retrospectively reviewed 6 patients (4 male, 2 female) with a median age of 10.6 years (range: 4.1 - 24.1 years) who had percutaneous closure of CAF from March 2010 - March 2012. The closure results and clinical follow-up were analysed.

Results: The origin sites of the fistulas were in the left $(n=3)$ and right $(n=3)$ coronary arteries. The fistulas drained to the right atrium $(n=3)$ and right ventricle $(n=3)$. I patient had multiple drainage sites to the right ventricle. A single device was used in 4 patients: vascular plug $(n=2)$, vascular plug $\|(n=1)$ and duct occluder II $(n=1)$. 2 patients required the use of more than I device (duct occluder and duct occluder II, 2 duct occluders with multiple coils). All devices were deployed retrogradely except in I patient. I patient had significant residual shunt at 6-month follow-up which was occluded by percutaneous technique. Followup studies 1.6 - 22.0 months (mean 13.9 months) showed complete occlusion in all patients.

Conclusions: Percutaneous closure of CAF is effective and safe with good results. With the availability of newer devices, most CAF can be closed percutaneously. Transcatheter closure should be considered as the treatment of choice.

\author{
Abstract no: 442 \\ of fetal aortic stenosis \\ Rudi Meyer ${ }^{\ddagger}$ and Klaus Schmidt ${ }^{t}$ \\ "Imperial College London, United Kingdom \\ \#Royal Brompton NHS Foundation Trust, London, United Kingdom \\ tHeinrich Heine University Düsseldorf, Germany \\ ¥Children's Heart Centre, Linz, Austria \\ \$Medical University of Warsaw, Poland \\ 'The Queen Silvia Children's Hospital, Gothenburg, Sweden \\ $\triangle$ Uppsala University Hospital, Uppsala, Sweden
}

Recognition of surgical outcome bias in multi-centre studies: A methodological approach using 109 cases

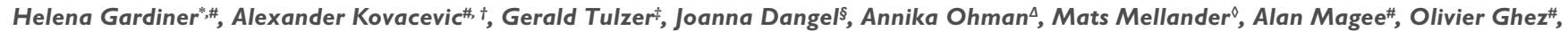

Background/hypothesis: The Fetal Working Group of the Association for European Paediatric and Congenital Cardiology studied the influence of fetal valvuloplasty on natural course of aortic stenosis (AoS) from fetal diagnosis to determination of surgical pathway.

Aim: To recognise bias, we tested a committee approach for the following hypothesis: institutional preference for neonatal AoS treatment overrides a committee's decisions based on morphological and clinical characteristics.

Material and methods: The committee re-assessed post-natal, pre-procedure imaging studies (including measurements and Z-scores) of 109 live born, prenatally diagnosed AoS (2005 - 20I I), treated in 24 institutions, blinded to fetal intervention, location and outcome.

Outcome measures for decision concordance: Individual and consensus decision on univentricular (UV) or biventricular (BV) pathway; concordance for first (BV) and second (UV) decision for those converting BV-UV; image quality; specialism (fetal/paediatric cardiologist, interventionalist or surgeon) and institutional ethos; Results: 64/109 had initial BV outcome, with later conversion to UV circulation in 6. Consensus concordance for first pathway: 85//09 (78\%); second: 81//09 (74\%). Concordance with initial BV surgical pathway in 4/ 6 required conversion to UV later. Poor imaging led to consensus "undecided" in 3/4. Committee decision was BV in 16/45 (36\%) final UV decisions and undecided in I (poor imaging). 
The interventionalist disagreed with I st $(p=0.015)$ and final outcome $(p=0.009)$, grading more UV than other specialists. Those with the greatest fetal intervention experience (GT, RM) agreed more with the Ist and final outcomes: ORI.86 (I.03,3.33; $p=0.039)$ and ORI.87 (I.04,3.38; $p=0.037)$. Committee's discordance was less for cases treated in surgical centres performing neonatal Ross-Konno ( I I\% vs. 27\%).

Conclusions: The committee's consensus was more optimistic than the eventual outcome, perhaps reflecting study members' experience in fetal intervention and neonatal Ross-Konno. This approach recognised greater discordance with institutions not performing Ross-Konno, leading to the potential to underestimate the influence of fetal valvuloplasty in achieving BV outcomes.

\section{Abstract no: 444 \\ Doppler echocardiographic diagnosis of fetal long QT syndrome with functional 2nd degree heart block}

\section{Sven-Erik Sonesson and Håkan Eliasson}

Karolinska Institute, Stockholm, Sweden

Background: A rare presentation of fetal long QT syndrome (LQTS) is a functional 2nd degree atrioventricular block $\left(2^{\circ} \mathrm{AVB}\right)$ sometimes in association with ventricular tachycardia leading to congestive heart failure. Junctional and/or ventricular tachycardia has also been suggested characteristic of an acute stage of antibody-mediated heart block requiring a completely different strategy of treatment. Recently observing a fetus with $2^{\circ} \mathrm{AVB}$ and abnormal diastolic relaxation, later confirmed to have LQTI, we speculated that this probably was an effect of a long refractory period in the ventricles and a possible marker to differentiate LQTS from other causes of $2^{\circ} \mathrm{AVB}$.

Material and methods: Isovolumetric relaxation (IRT) and contraction (ICT) time intervals were retrospectively determined from left ventricular inflow/outflow Doppler records obtained from 21 cases of fetal bradycardia: $2^{\circ} \mathrm{AVB}$ ( 5 with I LQTS), $3^{\circ} \mathrm{AVB}(7)$, and blocked atrial bigeminy (9).

Results: A markedly prolonged IRT ( $105 \mathrm{~ms})$ and a short ICT (7ms) clearly distinguished our LQTS case from all other cases, where IRT values ranged between $29-67 \mathrm{~ms}$ and short ICT values only seen in those with blocked atrial bigeminy. Long ICT values were seen in $3 / 4$ cases with antibody-mediated $2^{\circ} \mathrm{AVB}$.

Conclusions: Even if our observation of IRT prolongation in LQTS with $2^{\circ} \mathrm{AVB}$ is based on only one case, it is not unexpected and could well be explained by a QT-interval exceeding the duration of mechanical systole. Moreover, IRT prolongation was not seen in any other case with other mechanisms causing the fetal bradycardia, suggesting that measurements of IRT and ICT could be a valuable complement to get a correct diagnosis.

\section{Abstract no: 449 \\ Stent implantation in the aortic isthmus: An animal model of haemodynamic, hormonal, pressure and gene expression effects}

Ornella Milanesi, Nicola Maschietto, Luca Semplicini, Helen Poser, Giulia M De Benedictis, Giulio Ceolotto, Luca Aresu, Gabriele Gerardi, Ilaria lacopetti and Andrea Semplicini

University of Padua, Padua, Italy

Background: Stenting of the isthmus has been increasingly adopted for treatment of the aortic coarctation in children and adolescents. However it is not ascertained whether this technique can contribute to the development of late systemic hypertension.

Hypothesis: Stent implantation in the isthmus in growing subjects can affect haemodynamic, hormone incretion, arterial pressure and gene expression in an animal model.

Methods: Sheep 3 - 5 months-old underwent 2-D echocardiography, cardiac catheterization, haemodynamic study, and stent implantation in the isthmic region. Pressure was measured in the LV, ascending and descending aorta before and after stent implantation; sham animals underwent the same procedure without stent implantation. All were followed for one year to adulthood. Quarterly pressure measurements and echo-cardiography were performed; blood samples were drawn for determination of renine-angiotensin-aldosterone levels. A 2nd catheterisation, with haemodynamic study and a dobutamine test, was carried out I year after the enrolment, before the sacrifice. Samples were taken from myocardium, ascending and descending aorta for molecular biology examination.

Results: Four sheep received stent implantation, 4 sham animals were enrolled. The stent was oversized, considering the future growth. No differences in echocardiographic parameters or pressure measurements have been detected between the 2 groups in the interim evaluations. No pressure gradient across the isthmus was present at the follow-up; angiography did not show any narrowing. The dobutamine challenge test did not reveal differences, nor difference in renine-angiotensin-aldosterone level. The oxidative stress genes expression (MMP-9 and Caspase-3), showed a trend in significance in the expression of the MMP-9 that resulted higher in the ascending aorta of the animals with a stent.

Conclusion: Stent implantation in the in growing sheep does not affect haemodynamic, hormonal, pressure parameters. The increased expression of MMP-9 in ascending aorta of stented animals could indicate a subliminal endothelial dysfunction, promoted by the presence of a rigid metal segment.

\section{Abstract no: 45 I \\ Hematuria post cardiac catheterisation in children: Case series and literature review}

\section{Muhmmad Arif Khan, Omar Galal, Yahya Al Masham and Tarek Momenah}

Prince Salman Heart Centre, King Fahad Medical City, Riyadh, Saudi Arabia

Background: Mortality and morbidity from paediatric cardiac catheterisation procedures with or without interventions is very low. However, hematuria is a rare complication of cardiac catheterisation. This complication usually occurs from residual leak around the PDA coil.

Objective: To determine the frequency of hematuria in paediatric post catheterisation patients, its etiology and review of literature.

Methods and results: Retrospective study, review of all paediatric patients underwent cardiac catheterisation from January 2009 - July 2012 . All patients had routine blood investigations before the cardiac cath. Post cardiac cath all patients were observed for 24 hours before discharge. A total of 392 paediatric cardiac catheterisation procedures were performed. Total of interventions was 324(82.7\%), of those 60(I5.3\%) were PDA closure. Three (0.76\%) had Hematuria 2 male, I female age 8, 10, 14 months. Two of them had coil closure of the PDA with residual shunt. Third patient had hematuria secondary to hypercalciuria. Two patients were managed medically while third case recatheterised with retrievel of coil and replaced with device.

Conclusion: Hematuria is a rare complication of paediatric cardiac catheterisation. Patients need to be observed for hematuria. If it occurs should be managed and treated according to cause and severity. 


\author{
Abstract no: 489 \\ Melody pulmonary valve endocarditis \\ Juan Villafane*, Stephen Miller", G. Hamilton Baker ${ }^{\dagger}$ Lynn Peng ${ }^{\ddagger}$ and Erle Austin \\ "University of Kentucky, United States of America \\ \#Duke University Medical Center, United States of America \\ tMedical University of South Carolina, United States of America \\ ¥Stanford School of Medicine, United States of America
}

The Melody valve is an alternative to surgical replacement in selected patients with pulmonary outflow tract obstruction (PS) or regurgitation (PR). Implantable cardiac valves are susceptible to infection (SBE). We report on 4 male patients with Tetralogy of Fallot (TOF) who developed Melody pulmonary valve SBE, 3 of them required surgical valve replacement. In 2 the diagnosis was delayed. All survived.

\begin{tabular}{|c|c|c|c|c|}
\hline & Case I & Case 2 & Case 3 & Case 4 \\
\hline Age (years) & 8 & 14 & 20 & 11 \\
\hline RV outflow & Stentless porcine valve & Stented porcine valve & Bovine valve conduit & stented porcine valve \\
\hline Indication for Melody & PS-PR & PS & PS-PR & PS-PR \\
\hline Diagnosis of SBE & 3.5 months & 16 months & 7 weeks & I year \\
\hline Organism & Strep Viridans & Corynebacterium & Strep Sanguinis & Staph Aureus \\
\hline \multirow[t]{2}{*}{ Antibiotics } & Vanc $\times 8$ weeks & Pen G & Ceftriaxone $\times 8$ weeks & Nafcillin \\
\hline & Gent $\times 2$ weeks & $\times 7$ weeks & Gent $\times 6$ weeks & Rifampin, Gent \\
\hline $\mathrm{ECHO}$ & No changes & PS+ Vegetation & PS+ Vegetation & PS \\
\hline Surgical replacement & No & Yes & Yes & Yes \\
\hline
\end{tabular}

Case I had a tattoo a month prior to SBE. Cases 2 \& 3 had symptoms for several weeks while Case 4 presented with septic shock/ARDS requiring ECMO and emergency implantation of a second Melody valve for stabilisation.

Conclusion: The Melody valve can be subject to SBE. Therefore, prophylactic antibiotics during implantation and close post-implantation SBE surveillance are recommended. Three of our 4 patients presented with significant PS. Patients may present with a fulminant clinical course requiring aggressive management. Three of our 4 patients required surgical conduit replacement.

\title{
Abstract no: 492 \\ Impact of fetal echocardiography on patients' decisions in China: Preliminary report from a single centre in Shanghai
}

Lin Wu*, Luming Sun", Yingjun Yang", Gang Zou" and Guoying Huang*

"Children's Hospital of Fudan University, Shangai, China

\#Shanghai First Maternity and Infant Hospital of Tongji University, Wuhan, China

Background: Fetal echocardiography allows for pre-natal diagnosis of congenital heart disease or severe arrhythmia, and now serves as a routine screening tool for fetal cardiac anomalies in Shanghai. The aim of this retrospective study was to evaluate the impact of pre-natal echocardiography diagnosis on parents' decisions.

Materials and methods: A total of 112 patients were referred for detailed fetal echocardiography and prenatal counselling to a pre-natal counselling clinic in Shanghai First Maternity and Infant Hospital from July 2010 - December 201 I. The serial echocardiographic assessment was performed by both an experienced fetal sonographer and paediatric cardiologist. Based on the diagnosis of fetal echocardiography, a paediatric cardiologist, obstetrician and neonatologist will provide pre-natal counselling for the couples to outline the treatment options and to provide a true and clear picture of prognosis.

Results: Among these 112 patients 4 I fetuses (36.6\%) were diagnosed with congenital heart disease; 5 (4.5\%) with severe cardiac arrhythmia leading to haemodynamic compromise; and I with a huge cardiac tumour. With the above counselling, stillbirth was found in I patient. Abortion was opted for by 23 patients (56.1\%) with prenatally diagnosed congenital heart disease; 4 (80\%) with severe arrhythmia and in I with cardiac tumour, respectively. And abandoning treatment after birth in 2 patients, including I patient was diagnosed with transposition of great arteries with abnormal coronary arteries and I was congenital complete atrioventricular block with extremely low heart rate.

Conclusions: In China, most parents are not willing to accept multiple-staged surgical repair and possible long-term complication. The medical cost, neurodevelopmental outcome and ultimate quality of life, play important roles on parents' pre-natal decisions. 


\title{
Abstract no: 533 \\ Non-conventional uses of occluder devices
}

\section{Savitri Shrivastava}

Fortis Escorts Heart Institute (Fehi), New Delhi, India

Device closure is now an accepted modality of treatment for cardiac septal defects. We are reporting on the efficacy of closure of nonseptal defects with devices conventionally used for septal cardiac defects; A retrospective study.

Material and methods: 46 patients, age group 2 - 67 years were divided into 2 groups: Group l: with no available customized device; and Group 2: For which customized devices are available but alternate devices have been used. 37 in group I had: ruptured Sinus of Valsalva (duct occluder $n=I$ I); coronary arteriovenous (CAV) fistula (duct occluder: $n=5)$; closure of paravalvular leak $(n=5)$; mitral $(n=4$ : duct occluder devices=3,VSD device: $n=1$ ); and aortic $($ duct occluder: $n=1$ ); closure of AP window (duct occluder: $n=3$ ); Fontan fenestration closure ( $n=3$, asd septal occluder, patent foramen ovale device, vascular plug, I each); Pulmonary AV fistula (duct occluder; $n=2$ ); systemic AV fistula (vascular plug; $n=1$ ); closure of ascending aorta perforation (septal occluder, $n=1$ ); occlusion of subclavian artery (vascular plug; $n=1$ ); splenic artery (duct occluder; $n=1$ ); and Blalock-Taussig shunt (duct occluder, $n=1$ ). In group 2 there were 9 patients who had closure by ADO II device $(n=6)$, PDA closure by muscular VSD device $(n=2)$ and ASD device $(n=1)$.

Results: Residual shunt was detected in 2 patients each with coronary AV fistula and mitral paravalvular leak. No shunts were detected in the ruptured sinus of Valsalva, fenestrated Fontan and ascending aorta perforation. Complications: Local site haematoma (4); haematuria (4); I mortality in a large RSOV with gross congestive heart failure. On follow-up (2 months-6 years), all the patients are asymptomatic with no late complications.

Conclusion: It is feasible in selected lesions, to treat successfully with the use of non-prototype occluder devices without significant complications.

\section{Abstract no: 535}

Successful treatment of multiple mycotic aneurysms of aortic coarctation with covered stents

\section{Mehnaz Atiq", Imran Iftikhar\#, Ahmed Noor", Sajid Dhakam" and Zahid Amin ${ }^{\dagger}$}

"Department of Paediatrics and Child Health, Aga Khan University Hospital, Pakistan

\#Department of Cardiology, Indus Hospital, Karachi, India

tRush Medical Centre, Chicago, United States of America

Introduction: Coarctation of the aorta constitutes $7 \%$ of all congenital heart diseases and is 2 - 5 times more frequent in males. Infective endocarditis has been reported in $18 \%$ of aortic coarctation and the resulting mycotic aneurysm has a high mortality. The treatment reported in different case reports for these aneurysms has been surgical. We report a case of a long segment coarctation treated with multiple covered stents.

Case report: 22-year-old-female was investigated for hypertension and fever for 3 months. She was a known case of auto-immune disease with anaemia and moderate splenomegaly. Trans-thoracic echocardiogram revealed coarctation of aorta and transoesophageal echocardiogram showed multiple vegetations and aneurysms in the post stenotic segment. She was treated with ceftrioxone (for 4 weeks) and gentamycin (for 2 weeks) for streptococcus viridans. MRA revealed a long segment coarctation distal to the left subclavian with multiple aneurysms in the post stenotic area. Conventional aortogram confirmed the above findings. The pressure gradient across the coarctation was $100 \mathrm{mmHg}$. Two overlapping covered CP stents (Nu-Med, New York, USA) were placed to dilate the coarcted segment as well as completely cover the aneurysms, reducing the pressure gradient to $5 \mathrm{mmHg}$. The recovery was unremarkable.

Discussion and conclusion: Osler introduced the term mycotic aneurysm in 1885 to describe any aneurysm that develops in context with bacterial endocarditis irrespective of the pathogen. Mycotic aneurysm has a high risk of rupture in coarctation of aorta with up to 50 - $100 \%$ mortality. Treatment of adult coarctation with covered stent is a standard practice in several centres. The PTFE covering is useful in treating aneurysms complicating bare metal stent implantation or coexisting patent ductus arteriosus. This report highlights the safety of endovascular covered stents in managing mycotic aneurysms of coarctation of aorta not reported hitherto.

\section{Abstract no: 538 \\ An unusual systemic venous collaterals channel to left atrium causing desaturation after Fontan operation closed percutaneously}

Savitri Shrivastava, A. Marwah, S.I. Khatri and K.S. lyer

Fortis Escorts Heart Institute, Okhla Road, New Delhi, India

\begin{abstract}
We present an unusual cause of progressive cyanosis in a child two years after successful Fontan surgery due to I large venous channel and 2 small venous channels draining into LA directly. The larger channel was closed by Amplatzer vascular plug and small venous collaterals were closed by coils, resulting in improvement of oxygen saturation.

Case report: NS, a 7-year-old-girl a case of ToF with hypoplastic right ventricle and adequate branch pulmonary arteries had undergone modified right BT shunt in infancy followed by BD Glenn, ligation of pulmonary artery and takedown of BT shunt at 16 months; followed by an extra cardiac Fontan at $51 / 2$ years. She had a smooth post-operative course with saturation of 95\%. She had gradual return of cyanosis, exertional dyspnoea and easy fatigability since 6 months. Her saturation was $85 \%$ at rest and $78 \%$ after 10 minutes walking. TEE revealed a normal functioning Fontan circuit, normal ventricular function, and no AV valve incompetence, pericardial or pleural effusions. I venous channel was seen arising from the brachiocephalic vein. On cardiac catheterisation, intracardiac and Fontan pressures were normal. IVC angiogram revealed a large tortuous venous channel arising at T8-T9 level and draining into the left atrium $12 \mathrm{~mm}$ diameter, an additional small vein was seen connecting the larger venous channel to the left atrium. In nominate vein angiogram revealed a small venous channel communicating with the LA. These channels were occluded with $8 \mathrm{~mm}$ Amplatzer plug and 2 coils. Post-deployment angiograms revealed complete occlusion of all the venous channels, with $94 \%$ saturation. She has remained well at 6 month follow-up. We could not find a similar case report in English literature. Conclusion: Large venous channels draining directly into left atrium can be closed percutaneously. Long term follow-up is necessary.
\end{abstract}




\author{
Abstract no: $\mathbf{5 4 5}$ \\ Prenatally borderline left heart a problem at all? \\ Monika Kaldararova*, Peter Tittel", Andrea Kantorova*, Martin Culen*, Michal Holan" and Martin Zahorec* \\ "National Institute for Cardiovascular Diseases, Children's Cardiac Center, Slovakia \\ \#Medifera Medical Center, Slovakia
}

Background: In borderline left heart (Border-LH) the question of two vs. single-ventricle circulation arises. Aim of the study was a detailed analysis of pre-natal Border-LH and further post-natal outcome.

Patients and methods: 14 patients (9F/5M) with Border-LH identified prenatally from 2008 - 2012 were analysed. I. Pre-natally echocardiographs were measured: mitral/tricuspid valve (MV/TV) annulus, aortic/pulmonary valve (AOV/APV), left/right ventricular (LV/RV) width and length; were compared to 35 healthy matched controls (NORMAL) and to 17 patients with hypoplastic left heart syndrome (HLH). 2. Postnatal anatomy and outcome was evaluated, with median follow-up at 15 months.

Results: Pre-natally in border-LH significantly smaller structures compared were found to be normal but bigger compared to HLH. Border-LH/NORMAL/HLH: median MV/TV 0.60/0.92/0.42 ( $p<0.000$ I), AO/AP 0.54/0.9I/0.32 ( $<<0.000$ I), LV/RV width 0.59/0.93/0.42 ( $<<0.000$ I), LV/RV length 0.92/0.99/0.4I ( $<<0.000$ I). Post-natal Z-scores: median MV - I.2, AOV - I.05; smaller (Z-score <-2) MV and/or AOV in 3 patients (2I.4\%). Additional findings were: ventricular septal defect (VSD) in 7 (50\%), bicuspid aortic valve in 6 (42.9\%), persistent left superior vena cava to coronary sinus in 3 (2I.4\%) patients. All patients were able to maintain a biventricular circulation postnatally, I (7.1\%) w/o intervention. 13 (92.9\%) developed pathology, I0 (7I.4\%) underwent coarctation of aorta (COA) repair; I VSD closure. 3 (21.4\%) early deaths occurred: 2 prior surgery, I after COA repair. No late deaths were registered. By 2 months MV and AOV were all in normal range. In 2 (14.3\%) balloon angioplasty for re-COA was performed, in 2 (I4.3\%) mild mitral stenosis developed, w/o intervention needed.

Summary: Border-LH means thinner LV with almost normal length and smaller MV and/or AOV pre-natally; but with normal post-natal growth potential. Despite the positive consequence of 2-ventricular circulation, these patients are not free of interventions. COA can be expected with arterial duct closure. Frequently associated pathology indicates that this is not just pre-natally "lower-flow" circulation but that it probably represents a more complex morphological problem.

\title{
Abstract no: 563
}

Fetal pulmonary valvuloplasty by percutaneous trans-hepatic access in a lamb model

\author{
Andrew Edwards*,\#, ${ }^{*}$ Alex Veldman*, Ilias Nitsos*, Yuen Chan ${ }^{\S}$, Nadine Brew*, Samuel Menahem ${ }^{\dagger, 0}$, Dietmar Schranz ${ }^{\ddagger}$ and Flora Wong $, 0, \Delta$ \\ "The Ritchie Centre, MIMR, Monash University, Melbourne, Australia \\ \#Perinatal Services, Monash Medical Centre, Melbourne, Australia \\ †Fetal Cardiac Unit, Monash Medical Centre, Melbourne, Australia \\ ^Pediatric Heart Centre, Justus-Liebig University, Giessen, Germany \\ §Department of Pathology, Monash Medical Centre, Melbourne, Australia \\ 'Department of Paediatrics, Monash University, Melbourne, Australia \\ «Monash Newborn, Monash Medical Centre, Melbourne, Australia
}

Background/hypothesis: Fetal pulmonary valvuloplasty may ameliorate hypoplastic right heart syndrome and mitigate post-natal disease. Fetal heart access by direct fetal heart puncture is well-described. We have recently developed a percutaneous transhepatic fetal cardiac catheterisation technique, which may be safer and offer technical advantages. We hypothesised that fetal pulmonary valvuloplasty could be performed by a percutaneous transhepatic approach at midgestation.

Materials and methods: Nine fetal lambs at 97 - 100 (term 147) days gestation (average weight: I 215 grams) under maternal general anaesthesia were studied. Under ultrasound guidance, the fetal hepatic vein was percutaneously punctured using a I6GA IV-cannula with needle in-situ. A 2.4/I.9Fr coronary catheter was inserted over a 0.014 inch floppy guide wire, and the IVC, RA, RV, pulmonary artery, ductus arteriosus and descending aorta catheterised. After removing the guiding catheter, but with the guide wire still in place, a coronary PTCA dilatation catheter was positioned across the pulmonary valve, and several inflations of the balloon were performed simulating a valvuloplasty. 7 fetuses were euthanised post-procedure, and 2 were euthanised after term delivery for post mortem examination.

Results: Percutaneous cannulation of the fetal hepatic vein followed by RA and RV catheterisation was successful in all cases. I fetus died during catheterisation following RV perforation. In the remaining 8 cases the coronary catheter was advanced to the descending aorta. Pulmonary valvuloplasty was successful in 5 cases using catheters with a $6 \mathrm{~mm}$-long balloon: post mortem showing minimal haemorrhage without cardiac trauma. The procedure was unsuccessful in 2 cases (both survived) using a $12 \mathrm{~mm}$-long balloon which could not be turned into the pulmonary artery: post mortem showing small RV perforations. In I case the PTCA catheter could not be inserted as the cannula became dislodged.

Conclusions: Fetal pulmonary valvuloplasty by percutaneous transhepatic cardiac catheterisation is feasible, providing an alternative route for human fetal cardiac intervention.

\section{Abstract no: $\mathbf{5 7 2}$ \\ Spectrum and outcome of cardiomyopathies diagnosed during fetal life \\ Edgar Jaeggi*, Paul Kantor*, David Chitayat", Greg Ryan", Cedric Manlhiot* and Roland Weber* \\ "The Hospital for Sick Children, University of Toronto, Canada \\ \# Mount Sinai Hospital, University of Toronto, Canada}

Background: Primary cardiomyopathies (CM) are potentially irreversible, life-threatening heart muscle disorders that typically affect the cardiac filling and/or contractility in the absence of any anatomic abnormality. These are rare in-utero diagnoses and consequentially there is a paucity of knowledge on the entity. We sought to clarify the fetal disease spectrum and to identify early echocardiographic predictors of outcome.

Methods: We reviewed 53 consecutive cases with pre-natally detected CM at our tertiary care centre since 2000. Hypertrophic CM (HCM) was defined by the presence of ventricular wall thickness $>2$ Z-scores. Non-hypertrophic CM (NHCM) was defined by cardiac dysfunction in the absence of myocardial hypertrophy. Excluded were cases with cardiac hypertrophy secondary to maternal diabetes and twin-twin transfusion. 
Results: NHCM was diagnosed in 34 and HCM in 19 cases at 24.5+5.6 gestational weeks. Etiologies included familial (9\%), inflammatory (17\%) and geneticmetabolic disorders (36\%), while 38\% were idiopathic. The pregnancy was terminated in $9 / 53$ (17\%) cases. Of 44 actively managed cases, 20 (64\%) survived to infancy and 3/17 (I8\%) with HCM versus I4/27 (52\%) with NHCM remain alive (Hazard ratio (HR) 2.9; 95\% Cl 0.98-8.7; p=0.03). Baseline echocardiographic variables associated with mortality of actively managed cases included ventricular septal wall thickness (HR I.15 per 0Z-score increase; $95 \% \mathrm{Cl}$ I.03-1.29; $p<0.02$ ); fetal hydrops (HR 5.22; 95\% Cl I. I3-24. 16; $p<0.03$ ); umbilical vein pulsations (HR 9.5; 95\% Cl 2-45.3; $p=0.005$ ); $>2$ diastolic Doppler flow abnormalities (HR 3.54; 95\% Cl 0.98-12.77; $p<0.02$ ); left ventricular myocardial performance index (HR 2.6 per 10\% rise in index; $95 \% \mathrm{Cl}$ I-9; $\mathrm{p}<0.05$ ) and cardiovascular profile score (HR I.45 per deducted point; $95 \% \mathrm{Cl}$ I.10-1.90; p<0.0I).

Conclusions: Fetal CM is caused by a broad spectrum of causes and associated with significant peri-natal mortality, especially those with HCM. Early echocardiographic detection of diastolic dysfunction is most useful in predicting adverse outcomes.

\section{Abstract no: 574 \\ Percutaneous reconstructions of functional aortic interruption using perforation and covered stent}

Tarek Momenah*, Muhammad Khan*, Yahya Almasham*, Tarek Kashour", Mohammad Alhamadi and Nabeel Almashrki ${ }^{\dagger}$

"Prince Salman Heart Centre, King Fahad Medical City, Riyadh, Saudi Arabia

\#King Saud University, Riyadh, Saudi Arabia

tAlthourah Hospital, Sana, Yemen

Introduction: Coarctation of aorta is usually surgically treated in infancy and childhood, if untreated may lead to mortality. Rarely may result in acquired interruption.

Objective: Reconstruction of functional aortic interruption using perforation technique and covered stent.

Methods: 3 cases were suspected clinically and diagnosis confirmed by echocardiogram, cardiac CT and/or MRI. Cardiac catheterisation was done under general anaesthesia. Pre and post interruption areas were accessed by radial artery/transseptal puncture and femoral artery respectively. Pre and post interruption areas were assessed by angiograms. Transseptal needle $6 \mathrm{Fr}$ with dilator and sheath was advanced from femoral access and with aid of angiogram through other access. The needle was used to perforate the atretic part into the ascending aorta, position confirmed by pressure and angiogram. Then, with help of a PTCA (0.0 4 ) wire, the dilator and sheath were advanced and the sheath replaced by Mullens 12 Fr. After appropriate measurements, a covered stent was selected and mounted in BIB balloon taking in consideration the diameter of the transfer aortic arch and length to cover the whole atretic part of aortic arch and extended to descending aorta about 10 - $15 \mathrm{~mm}$ avoiding left subclavian artery origin. Post stent deployment pressure and angiograms were recorded.

Results: There were no early or late complications. All patients were discharged home within 3 days. Mean follow-up were 2 years and the gradient obtained were unchanged.

Conclusion: Percutaneous treatment of functional aortic atresia is a safe, feasible and effective alternative.

\section{Abstract no: 593}

\section{Fetal echocardiography in a developing country: Referral patterns and impact on pregnancy outcomes}

\section{Balu Vaidyanathan*, Deepa Sarala*, R.K. Kumar", John Simpson" and Gurleen Sharland"}

"AIMS, Kochi, India

\#Consultant, Evelina Children's hospital, London, United Kingdom

Background: Limited data exists about the practice of fetal echocardiography in developing countries with logistic limitations to treat complex congenital heart disease (CHD).

Objective: To report referral patterns for fetal echocardiography and its impact on outcomes of affected pregnancies in a newly established fetal cardiology unit in India.

Patients and methods: A fetal cardiology service was started in January 2008. Prospective data (2008 - 20 I 0 ) was collected in a dedicated database; retrospective data (2006 - 2007) was obtained from hospital records. Outcomes were tracked by direct evaluation of newborn, questionnaire or telephonic interviews.

Results: I 084 women were referred for fetal echocardiography; mean gestational age at referral was $24.9+5.9$ weeks. The most common indication for referral was abnormal screening ultrasound (38.4\%). Referrals for indications other than suspected CHD were more in the current period (2008 - 20I0) (63.8\% vs. 50.5\%; $\mathrm{p}=0.0006)$. CHD was diagnosed in 297 fetuses (27.5\%), I I 4 (38.4\%) were simple and I83 (6I.6\%) were complex. The most common outcome was termination of pregnancy (36\%) or intra-uterine/neonatal death (I8.9\%). Termination rates were higher for complex CHD (47\% vs. I8.4\%; $\mathrm{p}<0.000 \mathrm{I})$. Continuation of pregnancy with planned peri-natal care was more common in the current period $(25.5 \%$ vs. $10 \%$; $p=0.02)$ and for simple $\mathrm{CHDs}$ ( $36.4 \%$ vs. 14.2\%; $\mathrm{p}<0.00$ I).

Conclusions: Referral patterns for fetal echocardiography were characterised by late referrals, especially when CHD was suspected. Although half of the pregnancies affected with CHD resulted in termination or fetal/neonatal deaths, with improved awareness there was a trend towards planned deliveries and post-natal care, especially for repairable CHDs.

\section{Abstract no: 609}

Transcatheter device closure of perimembranous ventricular septal defects associated with aortic valve prolapse using Amplatzer duct occluder II device

\section{Muthukumaran Sivaprakasam, Archana Murugan, Neville Solomon, Prasad Manne and Ganapathy Subramaniam \\ Sri Ramachandra University, Chennai, India}

Aim: To report the feasibility of transcatheter device closure of perimembranous VSDs with aortic valve (AV) prolapse using an Amplatzer duct occluder II (ADO II): A single tertiary centre experience

Methods: Retrospective review of all ventricular septal defects with AV prolapse closed by transcatheter device closure from September 20 I I - July 20 I 2. The VSDs were closed by the retrograde technique using the Amplatzer duct occluder II (ADO II). 
Results: There were 4 patients with isolated VSDs associated with AV prolapse during the study period. Out of the 4, 3 were males and I female patient. They had an age range of $9-16$ years with a mean of 11.8 years and a weight range of $27.3-81 \mathrm{~kg}$. Three patients had a perimembranous defect and I had outlet extension. The size of the defect varied from $5 \mathrm{~mm}-6 \mathrm{~mm}$. Three patients had a mild LV dilatation while I patient had an associated aortic regurgitation. The procedure was successful in 3 out of 4 patients. One patient had device embolisation into the LPA, which was retrieved but got stuck to the Eustachian valve and has to undergo emergency surgery and the device was successfully retrieved intra-operatively. Post procedure echo in the 3 patients who had a successful device closure showed no worsening of AR, however, I patient had a tricuspid regurgitation. There was no residual shunt seen in any of these patients. Conclusion: Transcatheter closure of VSDs with AV prolapse is feasible with an ADO II device, but careful patient selection is mandatory as there is high rate of embolisation.

\title{
Abstract no: 618 \\ Transcatheter closure of perimembranous VSDs with duct occluders: Short term experience \\ Muthukumaran Sivaprakasam, Archana Murugan, Prasad Manne, Neville Solomon and Ganapathy Subramaniam \\ Apollo Children's Hospital, Chennai, India
}

Aim: To report the outcome of trans-catheter device closure of perimembranous VSD: A single tertiary centre experience.

Methods: Retrospective review of all VSDs closed by transcatheter device closure from September 20II - July 2012. The VSDs were closed either by the retrograde technique using the Amplatzer duct occluder II (ADO II) or the antegrade technique using the duct occluder I (DO I).

Results: Transcatheter device closure was attempted in 48 patients (24 females and 24 males) within the set time period. The age range was 1 I months 24 years, mean of 7 years, mode of 4 years and median of 6 years. The patients weight range was $7.9-81 \mathrm{~kg}$, mean of $22.2 \mathrm{~kg}$. The following anatomic types were present: 37 had perimembranous, 10 with inlet muscular extension and I subpulmonic. Other encountered associations were aortic regurgitation 3, AV prolapse 5 and tricuspid regurgitation 4. Median VSD size was $5 \mathrm{~mm}$ with a range of $4 \mathrm{~mm}-12 \mathrm{~mm}$. The mean procedure time was 35 minutes (range $25-100$ minutes) We used ADO II in 38 patients. Out of these I embolised in to the LPA. DO I was used in 10 patients. These patients had defects larger than $6 \mathrm{~mm}$. ADO $5 \mathrm{~mm} / 4 \mathrm{~mm}$ was used in 12 patients whereas ADO $6 \mathrm{~mm} / 4 \mathrm{~mm}$ was used in 26 patients. Procedure was successful in 47 patients (97.91\%). One patient had a device embolisation (ADO II) to the left pulmonary artery, which was retrieved but got stuck with the Eustachian valve and so he underwent emergency surgical retrieval of device with VSD surgical closure. Out of 5 patients with AV prolapse, I patient had AV prolapse with Aortic regurgitation, which remained the same and none had new onset AR. Tiny residual VSD was seen in 7 patients and all of those VSDs were closed by ADOII. None of the DO I patient had residual VSD. None of the patients developed Complete Heart block.

Conclusion: Trans-catheter closure of congenital VSDs offers encouraging results. More experience and long-term follow-up are mandatory to assess safety and effectiveness of this procedure as an alternative to conventional surgery.

\section{Abstract no: 619}

\section{The Edwards Valeo Lifestent ${ }^{\circledR}$ for treatment of cardio-vascular lesions in children}

\author{
Caroline Ovaert", Debora Luciano*, Anass Assaidi", Issam Kammache*, Bernard Kreitmann", Loic Mace" and Alain Fraisse* \\ "Paediatric cardiology, Timone Enfant AP-HM, Marseille, France \\ \#Paediatric cardiac surgery, Timone Enfant AP-HM, Marseille, France
}

Background: The Edwards Valeo Lifestent ${ }^{\circledR}$ is a stainless steel, pre-mounted, open cell stent. Easy dilation to larger diameters and low profile are advantages in growing children. Radial force is however poor.

Results: From April 20II - July 20।2, 26 Vale ${ }^{\circledR}$ stents were placed in 22 children during 23 procedures. Median age was I year (5 days - I4.3 years), median weight $8 \mathrm{~kg}(2$ - 53). Most insertions (87\%) were percutaneous (group I) while 3 were per-operative (group 2). Indications and locations were: RPA or LPA (rehabilitation strategy) in PA-IVS in 6; LPA stenosis in Fallot; PA-IVS or VSD in 4 (3 per-operative); PA stenosis in single ventricle in 2; PA stenosis after arterial switch in 2; sub-hepatic vein thrombosis in I; PDA stenting (hybrid approach) for HLHS in 6; and for complicated IAA in 2 patients. In group I, stent placement was straightforward, through femoral venous access in all except 3 (2 jugulars, I transhepatic). Pre-dilation was performed in 5, all with PA stenosis; subsequent post-dilation was performed in 2. Immediate angiographic and/or haemodynamic results were considered satisfactory in all. Acute complications occurred in 3 (haemoptysis in 2, reperfusion oedema in I). Six patients have so far been re-catheterised, a median of $1.7 \mathrm{mo}$ after initial procedure. The stent remained fully patent in all but 1 . In group 2, the stents were always secured with a single proximal stitch and flared at their proximal end. All 3 patients had early post-op re-catheterisation (median 6 months). All stents were re-dilated to achieve better wall apposition. In I a possible stent fracture without obstruction was seen. Conclusion: The Valeo ${ }^{\circledR}$ stent is an easy and effective stent for use in growing children. Radial force was sufficient in the lesions we encountered. When used per-operatively, early re-catheterisation is warranted to improve wall apposition. Longer-term follow-up is needed.

\section{Abstract no: 628 \\ Haemoptysis after fenestrated extracardiac Fontan: A multi-disciplinary approach to management}

\section{Usha Pratap, Prashant Mishra, Mukul Mutatkar, Vinayak Desurkar and Ranjit Jagtap}

Deenanath Mangeshkar Hospital, Pune, India

Background: Haemoptysis after fenestrated Fontan repair for univentricular physiology is a well known complication of chronic cyanosis. We present our management of a 10-year-old who presented with massive haemoptysis 5 years after completion of an extracardiac Fontan.

Summary: TD underwent a superior cavopulmonary anastomosis at age 2 for his transposition of great arteries with a small LV and PS. He had a fenestrated extracardiac Fontan done at age 5. At age 10 he presented with sudden onset haemoptysis. After stabilisation he underwent a right heart catheterisation to delineate the conduit-anatomy; an aortic DSA (Digital Subtraction Angiogram) was also performed to delineate possible bronchial collaterals. Right heart catheterisation revealed a $7 \mathrm{~mm}$ conduit-RA fenestration while the aortic DSA revealed multiple and large bronchial collaterals. Our interventional radiologist used 300-500 PVA (poly-vinyl alcohol) particles to successfully embolise the bleeding collaterals. We planned to close the large conduit-fenestration at a staged 
setting with an ASD occlusion device. Angiography and transoesophageal echocardiogram (TEE) revealed an anatomy which favoured using an Amplatzer Duct Occluder - 2 (ADO-2, St Jude Medical) device for closing the fenestration. This was successfully achieved using an ADO-2 6-6 device under fluoroscopy and TEE guidance. Post procedure angiogram revealed no right-to-left shunting. Pressure in the venous-PA conduit prior to device closure was II/5mmHg and postprocedure was 10/5 $\mathrm{mmHg}$. Arterial blood gas oxygen saturations improved to $96 \%$. He remains well and fully saturated 3 months later.

Conclusion: We would like to report this case since using a multi-disciplinary approach helped to reduce procedure and radiation time in a haemodynamically unstable patient. It was also a novel use of PVA particles for successful embolisation of the bronchial collaterals (with possible advantages over coil occlusion).

There have been no reports of using of ADO-2 device for percutaneous closure of the fenestration.

\title{
Abstract no: 638 \\ Balloon valvuloplasty in premature infants with severe aortic and pulmonary valve stenosis or pulmonary atresia
}

\section{Michael Rigby, Margarita Bartsota, Alan Magee and Zdenek Slavik}

Royal Brompton Hospital, London, United Kingdom

Background: The rationale for fetal balloon valvuloplasty is to prevent the development of left or right ventricular hypoplasia in the setting of severe aortic, pulmonary stenosis or pulmonary atresia intact septum.

Material and methods: A retrospective review of premature infants, weight $<2 \mathrm{~kg}$, gestation $<36$ weeks undergoing balloon valvuloplasty was performed.

Results: There were 6 with severe aortic stenosis, gestational age 32 - 36 weeks birth weight 1.4 - $1.9 \mathrm{~kg}$ treated in the first 10 days of life: 9 with severe/critical pulmonary valve stenosis, gestational age 28 - 36 weeks birth weight 1.2 - $1.9 \mathrm{~kg}$ were treated during the first 9 days; 3 with pulmonary atresia, weights 850 , I 700 and I 900 grams, had radiofrequency assisted balloon pulmonary valvuloplasty; 3 with aortic stenosis developed transient loss of a femoral artery pulse. The systolic gradient or left ventricular systolic pressure fell in every case; I developed severe aortic regurgitation and required a Ross operation at 2 months; I patient required repeat aortic valvuloplasty at 6 months; 2 had a Ross operation at 5 and 7 years respectively; I patient reached nine years without a 2 nd intervention.

Relief of pulmonary stenosis occurred in every case. I had repeat pulmonary valvuloplasty 12 months, but no other patient has required re-intervention 6 months to 12 years following the initial valvuloplasty. Of 3 patients with pulmonary atresia I born at 27 weeks died 10 days later from non cardiac causes. The other 2 did not require an additional source of pulmonary blood supply and have required no further intervention at 8 months and I year later.

Conclusions: Balloon valvuloplasty is a safe procedure in premature infants. Elective premature delivery before 36 weeks gestation with post-natal balloon valvuloplasty could be an alternative to fetal intervention in selected cases.

\section{Abstract no: 689}

\section{Pre-natal echocardiographic findings in hypoplastic left heart syndrome with coronary fistula}

\author{
Anna Lamberti", Annabelle Azancot*, Maurice Guirgis*, Andre Denjean*, Constance Beyler* and Damien Bonnet* \\ "Robert Debre Hospital, Paris, France \\ \#Universite Paris Descartes Sorbonne, Paris, France
}

Background: Pre-natal diagnosis of hypoplastic left heart is commonly performed during fetal life. Identifying risk factors to better predict outcome is still challenging.

Case report: We describe here the pre-natal echocardiographic features of a rare association of hypoplastic left ventricle with coronary fistula. Fetal echocardiography at 33 weeks of gestation showed a small left ventricle (LV) of $6 \mathrm{~mm}$ with mitral hypoplasia. The LV had a globular appearance with filled apex and a poor systolic function. A small $1.5 \mathrm{~mm}$ aorta arose from the LV and a biphasic Doppler flow was recorded in the aortic root suggesting aortic regurgitation. The ductus arteriosus perfused the horizontal and ascending aorta. The pulmonary artery diameter was $8 \mathrm{~mm}$. The delivery was planned in a tertiary centre. In the delivery room, Apgar was 5/8 and evidence for low cardiac output was already noted. Prostaglandin perfusion was started. Echocardiography showed a large left coronary fistula filling the LV. This was confirmed by a CT angiography. Subsequently, compassionate care was planned and the child died. In pre-natal echo, the LV cavity size was overestimated because the LV was filled by the coronary fistula. In addition, this fistula was responsible for a steal phenomenon that further compromised LV function while no endocardial fibrosis could be evidenced.

Conclusion: Coronary fistula is a rare associated lesion in a hypoplastic left ventricle. Awareness on this association might be useful to better predict post-natal outcome and to improve pre-natal counselling.

\section{Abstract no: 695}

Intra-cardiac echocardiographic guidance for transcatheter closure of atrial septal defect in an unselected adult patient population

\author{
Alain Fraisse, Marion Sumian, Anass Assaidi, Julien Mancini, Issam Kammache, Caroline Ovaert and Gilbert Habib \\ La Timone Hospital, Marseille, France
}

Background: Intracardiac echocardiography (ICE) is an alternative to transoesophageal echocardiography (TEE) for transcatheter closure of ostium secundum atrial septal defect (ASD). However, studies on ICE guidance generally include a majority of patent foramen ovale and only few ASDs. Our aim was to assess transcatheter closure of ASDs under ICE guidance in an unselected patient population, paying particular attention to complex cases with large (>38mm) ASDs and/or deficient rims other than the anterior-superior.

Material and method: From January 2006 - January 2012 in our centre, all the ASDs in the adult population were closed percutaneously under ICE guidance, except for 3 patients with Down's syndrome who underwent closure under general anaesthesia and TEE guidance. During this period, 93 patients (59 females) underwent transcatheter ASD closure at a median age of 48 (2I - 90) years with Amplatzer devices under local anaesthesia and ICE guidance. All patients had routine TEE before catheterisation. 
Results: Complex cases comprised 17 patients (18\%) including 13 with one or more deficient rims other than the anterior-superior and 8 with an ASD size $>38 \mathrm{~mm}$. The median ASD diameter by TEE and ICE was $20(6-40) \mathrm{mm}$ and 25 ( 10 - 40) mm, respectively. 90 cases (97\%) were successfully closed. Three cases failed because of insufficient rims and/or defect size superior to $40 \mathrm{~mm}$. Minor and transient complications (atrial arrhythmia, groin haematoma) occurred in 10 patients (9.6\%). Three patients experienced a major complication with favourable outcome: I arterial tear (laceration of a branch of hypo gastric artery) during venous puncture attempt successfully treated by percutaneous embolisa-tion, I blood transfusion for a groin hematoma and I retroperitoneal haematoma. The only risk factor for failure to close the ASD was deficient rims $(p=0.05)$.

Conclusion: In an unselected adult population, ICE provides a safe and efficient guidance for device closure of ASD, even for large defects with deficient rims.

\title{
Abstract no: 706 \\ Transcatheter closure of secundum atrial septal defect associated with deficient rims other than the antero superior
}

\section{Alain Fraisse, Issam Kammache, Anass Assaidi, Julien Mancini, Deborah Luciano and Caroline Ovaert}

La Timone Hospital, Marseille, France

Background: We aimed to assess the feasibility of transcatheter closure for secundum atrial septal defects (ASDs) with deficient rims ( $<5 \mathrm{~mm})$, other than the antero-superior because it is well demonstrated that deficiency in the anterior rim toward the aorta does not influence the success rate for transcatheter ASD closure.

Material and method: From January 2008 - December 201I, 192 patients underwent percutaneous closure of ASDs in our institution, using transoesophageal echocardiography guidance in children and intracardiac echocardiography guidance in adults. Amplatzer devices (Amplatzer Septal Occluder or Cribriform) were used in 191 whereas I case without deficient rim was closed with intrasept ASD occluder. We retrospectively analysed the outcomes of the 43 patients (22,4\%, 26 children) with one or more deficient rim.

Results: The median age and weight was $16(1,1-85)$ years and $56(8,8-99) \mathrm{kg}$, respectively. Deficiency of the inferior $(n=11)$, inferior-posterior $(n=15)$, or the superior-posterior rim $(n=17)$ was confirmed by transoesophageal echocardiography in all the cases. Transcatheter closure was successfully accomplished in 37 (86\%) of the cases with a median Amplatzer Septal Occluder size of 28 ( $10-40) \mathrm{mm}$. A modified method of implantation (sizing balloon technique) was used in 30 patients (70\%). In 6 patients (5 children) the ASD could not be closed. Four other children experienced device embolisation a few hours after successful transcatheter closure and underwent surgical ASD closure without further complications. By Fisher's exact test, deficiency of the inferior-posterior rim was associated with failure or embolisation $(p=0.05)$ and there was a trend for adult age to be associated with a low risk of embolisation or failure $(p=0.06)$. Conclusion: Transcatheter closure of the secundum ASD is feasible in patients with deficient rims. However, embolisation may occur especially in the paediatric population with deficient inferior-posterior rim.

\author{
Abstract no: 712 \\ Early results from the United States Melody ${ }^{\circledR}$ transcatheter pulmonary valve: A post-approval study

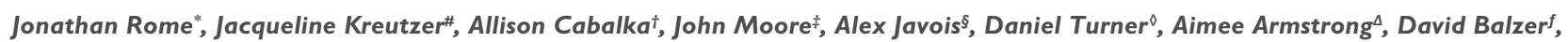 \\ Collin Cowley ${ }^{\vee}$ and Thomas Zellers' \\ "Children's Hospital of Philadelphia, University of Pennsylvania, Philadelphia, United States of America \\ \#Children's Hospital of Pittsburgh, Pennsylvania, Philadelphia, United States of America \\ †Mayo Clinic, Rochester, Minnesota, United States of America \\ \#Rady Children's Hospital, San Diego, California, United States of America \\ ^Advocate Hope Children's Hospital, Oak Lawn, Illinois, United States of America \\ ${ }^{\circ}$ Children's Hospital of Michigan, Detroit, Michigan, United States of America \\ $\Delta$ University of Michigan Congenital Heart Centre, CS Mott Children's Hospital, Ann Arbour, Michigan, United States of America \\ ${ }^{f}$ St. Louis Children's Hospital, St Louis, Missouri, United States of America \\ 'Primary Children's Medical Centre, University of Utah, Utah, United States of America \\ University of Texas South-western Centre, Dallas, Texas, United States of America
}

Background: This study was undertaken to evaluate short-term outcome after percutaneous transcatheter pulmonary valve (TPV) implantation in a "real-world" clinical setting. The primary outcome is TPV function and freedom from reintervention at 6 months. Here we present procedural and early results.

Methods: Implantation procedures were based on IFU guidelines. Data were collected prospectively on all consecutive subjects enrolled. Procedural success was defined as TPV appropriately fixed in position; catheter gradient $<35 \mathrm{mmHg}$; and strivial pulmonary regurgitation by angiography. Successful TPV function at endpoint was mean echocardiographic gradient $\leq 30 \mathrm{mmHg}$ and mild or less regurgitation.

Results: From July 2010 - May 2012, 127 patients were enrolled at IO U.S. centres for possible TPV implantation at a mean age of 20.0 99.7 years. The primary indication was conduit stenosis in 19.8\%; regurgitation in $42.9 \%$; and both in $30.2 \% .78 \%$ of patients were NYHA I or II. Most conduits were homografts (65.9\%); 12.7\% were bio prosthetic. TPV implant was attempted in 98 patients, 97 (99\%) were successful. Procedural success rate was 93\% (90/97) with no mortality. Serious procedure-related events included: conduit rupture ( $\mathrm{N}=6$; all treated with covered stents, 5 underwent TPV, I surgery); pulmonary artery perforation ( I); and coronary compression requiring emergency surgery ( 1 ). At discharge, the average mean gradient was $16.9 \pm 7.6 \mathrm{mmHg}$; $97 \%$ had no or trace pulmonary regurgitation. At mean follow-up of 7.7+6 months 2 TPV were explanted, I for endocarditis and I during aortic root replacement. Another patient was treated for presumed endocarditis.

Conclusion: These early data suggest TPV in standard clinical practice yields procedural results equivalent to those in the IDE study. The procedure appears an acceptable alternative to surgical conduit replacement. Endocarditis risk warrants further study. 


\title{
Abstract no: 735 \\ Atrial level restriction influences cerebrovascular impedance in the fetus with transposition of the great arteries
}

\section{Jack Rychik, Hilea Su and Zhiyun Tian}

The Children's Hospital of Philadelphia, Pennsylvania, United States of America

Background: In transposition of the great arteries (TGA) the aorta arises from the right ventricle, which leads to alteration in utero of cerebral blood flow and oxygen delivery. Restriction at the atrial septum requires balloon atrial septostomy (BAS) after birth, but may also influence patterns of cerebral blood flow before birth.

Objective: To characterise cerebrovascular impedance in the fetus with TGA and investigate its relationship to atrial septal restriction.

Methods: Fetuses with TGA, intact ventricular septum, 2 good-size ventricles and no outflow tract obstruction had middle cerebral artery pulsatility indices (MCA-PI) measured and compared to normal gestational age-matched controls. TGA group was divided into BAS and no-BAS after birth, reflecting the degree of pre-natal atrial restriction.

Results: Thirty six fetuses with TGA (17=no BAS; 19= BAS) were compared to 126 controls. Overall there was no difference in MCA-PI between TGA and normal. Comparing cohorts of similar gestational age, at the end of gestation (36 - 39 weeks) the BAS group had MCA-PI similar to normal, which was significantly lower than that of the no BAS group (BAS I.52+0.07 vs. no BAS $2.02+0.15, p<0.01$ ).

Conclusions: MCA-PI normally decreases at the end of gestation (36-39 weeks). MCA-PI is normal in the fetus with TGA and a restrictive atrial septum, but is abnormally elevated near the end of gestation when there is an open atrial septum. We speculate that a restrictive atrial septum leads to increased mixing in the right atrium, with cerebral oxygen delivery that is closer to normal, while under conditions of open atrial septum, streaming leads to severely deoxygenated blood delivery to the brain. Chronic cerebral hypoxia in TGA with open atrial septum leads to cerebrovascular dysregulation with markedly elevated MCA-PI. Childhood neurocognitive deficits in TGA may in part have their origins in pre-natal life as a consequence of variability in cerebral blood flow and oxygen delivery.

\author{
Abstract no: 753 \\ and Christine Houde ${ }^{\dagger}$ \\ "Sainte-Justine Hospital, Montreal, Quebec, Canada \\ \#Montreal Heart Institute, Montreal, Quebec, Canada \\ †Laval University Hospital, Quebec, Canada

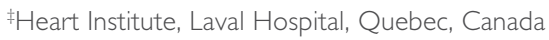 \\ ${ }^{\S}$ Children's Hospital, Montreal, Quebec, Canada
}

Are covered stents protective against acute local complications in high-risk patients with aortic coarctation?

Baher Hanna*, Sylvia Abadir", Frederic Dallaireł, Josep Rodes-Cabau", Joaquim Miro*, Adrian Dancea§, Reda Ibrahim", Nagib Dahdah*

Background: Percutaneous dilatation and stenting of aortic coarctation (CoAo) has proved to be an effective alternative to surgery. Because of complications associated with bare metal stent (BMS) such as local dissection, covered stents (CVS) were introduced.

Aim and hypothesis: To compare the results and complication rate between BMS and CVS in the treatment of CoAo. We tested the hypothesis that a 50\% or more stenosis in relation to the aorta at the diaphragm level is a significant risk factor associated with BMS for acute local complications including aneurysm formation and bleb tears.

Materials and methods: Retrospective chart review of patients treated at the collaborating centres, comparing pre and post procedural data and procedural details. Patients who received BMS with stenosis $\geq 50 \%$ were considered high-risk (HR-BMS). The 2 BMS groups were compared to a group of patients with stenosis who CVS $\geq 50 \%$. Acute complications were classified as "in situ" (stent migration, intimal tear, and aortic aneurysm) or major (death, stroke, wall rupture, cardiac arrest, transfusion, and necessity of urgent reintervention). Early and mid-term MRI studies were reviewed for potential in situ complications.

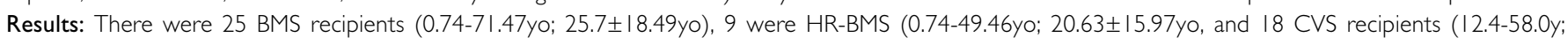
$26.74 \pm$ |4.63y). Early total complication rate was $9 / 25$ (36\%) in the BMS group, and 6/9 (66.6\%) in the HR-BMS compared to 4/I8 (22.2\%) in the CVS group $(p=0.503$ and 0.108 respectively). Major complications occurred in 3/25 (I2\%) BMS patients, and in 3/9 (33.3\%) HR-BMS, compared to I/I8 (5.56\%) CVS, $(p=0.628$ and 0.093 respectively). In situ complications were encountered in 3/25 (I2\%) BMS patients, or I/9 (I I. I\%) HR-BMS, compared to 0/I8 (0\%) CVS patients; $(p=0.25$ and 0.33 respectively). Finally, the re-catheterisation rate was $6 / 25$ (24\%) for BMS, and 4/9 (44.4\%) in the HR-BMS compared to $3 / 18$ (I 6.67\%) in $\mathrm{CVS}$; ( $\mathrm{p}=0.7 \mathrm{I}$ and 0.175 respectively).

Conclusion: Based on our limited series, the use of covered stents did not significantly reduce the risk of early in situ complications. Long term follow-up imaging is needed for appropriate assessment of the hypothetical risk of long term aortic wall integrity following CoAo stent procedures.

\section{Abstract no: 774 \\ Profile and pregnancy outcomes of fetuses with conotruncal anomalies in a newly established fetal cardiology unit in South India}

Balu Vaidyanathan, Shine Kumar and R.K. Kumar

Amrita Institute of Medical Sciences (AIMS), Kochi, India

Objective: To describe the profile and immediate pregnancy outcomes of fetuses with conotruncal anomalies (CTA) in a newly established fetal cardiology unit in South India.

Methods: Records of 68 women diagnosed with CTA on fetal echo (mean gestational age $26.75 \pm 5.93$ weeks; range 17 - 38 weeks) during the period 2008 201 I were reviewed.

Results: The most common indication for referral was suspected CHD during routine ante-natal scan (89.7\%). The various CTA diagnosed included: Tetralogy of Fallot (ToF, 44.I\%); double outlet RV (DORV, 27.9\%); transposition of great vessels (TGA, 8.8\%); ToF with pulmonary atresia (ToF-PA, 8.8\%); ToF absent 
pulmonary valve (ToF-APV, 7.4\%); and truncus arteriosus (2.9\%). Extra cardiac anomalies were reported in 4 fetuses (7.1\%). Pregnancy outcomes were reported for 65 fetuses (95.6\%), with 4 being lost to follow-up. The most common outcomes included: termination of pregnancy (4I\%); planned delivery and post-natal care (4I\%); intra-uterine death (13\%); and neonatal death (5\%). Termination of pregnancy rates based on individual diagnosis was as follows: ToF (43.3\%); DORV (42. I\%); TGA (50\%); ToF-PA ( $6.7 \%)$; ToF-APV (40\%); and truncus arteriosus (50\%). Overall post-natal survival amongst patients with intention to treat was $86.9 \%$ with 5 patients undergoing surgery in the neonatal period. The accuracy of fetal echo in identifying the primary diagnosis was $96.4 \%$.

Conclusion: Pre-natal diagnosis of conotruncal anomalies prompted post-natal care in less than half of the diagnosed patients with a very high proportion of even reparable CTAs undergoing termination of pregnancy. Post-natal survival of those with intention to treat was excellent.

\title{
Abstract no: $\mathbf{8 0 3}$
}

Transcatheter closure of perimembranous ventricular septal defect using Amplatzer ductal occluder

\section{Seong-Ho Kim*, Sang Mi Lee", Jae Young Choi", Jin Young Songt, Sang Yoon Lee*, Jae Sook Paik*, So Ick Chang* and Woo Seop Shim*}

"Sejong General Hospital, Korea

\#Yonsei University Hospital, Seoul, Korea

tSamsung Medical Centre, Seoul, Korea

Background: Transcatheter closure of perimembranous ventricular septal defect (PMVSD) is a challenging procedure. Recently, the Amplatzer PMVSD occluder (AGA Medical Corp, Plymouth, Minnesota) has been shown to be effective in closing haemodynamically significant PMVSDs. However, the high incidence of complete atrioventricular block (CAVB) after device occlusion of a PMVSD using it has been a hot issue, too. Among several devices tried to close PMVSD percutaneously, we prefer to use the Amplatzer ductal occluder (ADO, AGA Medical Corp, Plymouth, Minnesota) because of the anatomic resemblance between PMVSD with aneurysm and patent ductus arteriosus.

Materials and methods: Between August 2009 and June 2012, 21 patients underwent percutaneous PMVSD closure using ADO: 5 males and I6 females, with median age of $6(6-42)$ years. Their median weight was $23(18-60) \mathrm{kg}$.

Results: All patients showed echocardiographic signs of left ventricular volume overload and trivial to small mitral regurgitation $(Q p / Q s=1.7 \pm 0.4)$. The mean defect sizes of the right ventricular side were $4.7 \pm 0.8 \mathrm{~mm}$. Devices $2 \mathrm{~mm}$ larger than the measured narrowest VSD diameter were selected in most patients. The ADOs were successfully implanted in all patients without any significant complications except one transient CAVB and one case of delivery wire fracture. Small residual shunts were observed immediately after device implantation, but have disappeared during follow-up in 20/2I patients. Mean follow-up period was $18 \pm 8$ months, and CAVB or aortic regurgitation was not observed in all patients.

Conclusions: Transcatheter closure of perimembranous VSD with the Amplatzer ductal occluder is a safe and promising treatment option, but long term followup in a large number of patients would be warranted.

\author{
Abstract no: 808 \\ Using fetal echocardiography in the evaluation and treatment of fetal anaemia \\ Darren Hutchinson*, Lisa Hornberger*, Venu Jain", Sue Chandra", Yuka Yamamoto* and Akiko Hirose* \\ "Stollery Children's Hospital, Edmonton, Alberta, Canada \\ \#Lois Hole Hospital for Women, Edmonton, Alberta, Canada
}

Background: Significant fetal anaemia can cause fetal compromise due to high fetal cardiac output (CO), with intrauterine transfusion (IUT) being the treatment of choice. IUT carries risks including pre-term delivery and fetal demise. Peak middle cerebral artery (MCA) Doppler velocity > I.5 multiples of the mean (MoM) is sensitive for moderate-to-severe fetal anaemia with false positive rates of $\geq 12 \%$. We sought to explore the additive value of FE and calculated fetal combined $\mathrm{CO}(\mathrm{CCO})$ in assessing fetal anaemia.

Methods: We identified pregnancies that underwent FE prior to IUT for suspected fetal anaemia from June 2009 - June 2012 in our programme. FE was used to calculate fetal CO. Prenatal records were reviewed for peak MCA velocity at IUT, pre-transfusion haemoglobin (Hb) and hydrops. Anaemia was graded as moderate if fetal $\mathrm{Hb}$ was $<0.65 \mathrm{MoM}$ and severe if $<0.55 \mathrm{MoM}$.

Results: Eleven pregnancies complicated by fetal anaemia had 20 IUTs. 9/ I I pregnancies had FE within the preceding 30 days. One case was excluded due to no pre-transfusion $\mathrm{Hb}$ measurement. Median gestation at FE was 24 weeks (range I 9 weeks - 29 weeks). Peak MCA Doppler was > I.5MoM (range I.5 - 2.5) in all. Of the 9 fetuses with measured Hb; $44 \%$ had severe ( $n=4,3$ of whom with hydrops); $22 \%$ moderate ( $n=2)$; and $33 \%$ ( $n=3$ ) mild anaemia. Fetal CO was above the 90th percentile in all fetuses with moderate or severe anaemia. In 3 with mild anaemia, two had normal $\mathrm{CO}$ and one had $\mathrm{CO}$ at the 90 th percentile. Conclusions: FE-derived $\mathrm{CO}$ may be a useful additional tool in the evaluation of fetal anaemia severity. A normal fetal CO, even with elevated peak MCA Doppler, may allow the clinician to predict mild anaemia at fetal blood sampling. Surveillance using FE may avoid or delay the need for invasive management and the associated fetal morbidity and mortality.

\section{Abstract no: 81 I \\ Ease of retrievability of ADO II: An added advantage}

Premsekar Rajasekaran, Anurad ha Sridhar, Saileela Rajan and Raghavan Subramaniam

Frontier Lifeline Hospital \& Dr K.M. Cherian Heart Foundation, Chennai, India

Background: Amplatzer Duct Occluder II (ADO II - AGA Medical Corporation, Plymouth, MN 55442, USA) has found wide favour in transcatheter closure of large ductal shunts as well as in retrograde transcatheter closure of perimembranous and muscular VSDs. This is attributable to the fabric free design and thinner Nitinol wire mesh rendering device delivery through smaller catheters feasible. However, this design also enhances the flexibility of the device making it prone to deformation and migration when deployed across large $(>5.5 \mathrm{~mm})$ high pressure shunt lesions, despite optimum placement of the device.

Materials and Method: We report our institutional experience of ADO II from August 20 I I - date, wherein 27 devices were implanted (Perimembranous VSD 19, Patent Ductus Arteriosus 6, Muscular VSD I, Aortopulmonary window I in 27 patients aged 4 months - 12 years (mean age 4.7 years) with 3 devices 
migrating to the pulmonary artery. One was in an infant with a $5.5 \mathrm{~mm}$ Type A PDA closed with 6-4 ADO II and the other 2 were children with $5 \mathrm{~mm}$ perimembranous VSDs where a 5-4 ADO II was deployed. The retrieval was done by Ist positioning a 7 Fr Mullins sheath in the distal main pulmonary artery through which a $5 \mathrm{Fr}$ snare catheter and $5 \mathrm{~mm}$ gooseneck snare (Cook incorporated, Bloomington, USA) is advanced to the embolised device. The proximal screw of ADO II was snared and the device withdrawn into the 7 Fr Mullins sheath.

Results: In all 3 cases, the devices were retrieved successfully and subsequent transcatheter closure performed with a Nitinol duct occluder.

Conclusion: ADO II is not ideal for use in high pressure shunt lesions measuring $>5.5 \mathrm{~mm}$. However, the fabric free design and the thinner Nitinol wire mesh renders it easily retrievable even after being completely deployed.

\author{
Abstract no: 832 \\ Ten year's experience of transcatheter closure of ventricular septal defect in Jakarta, Indonesia \\ Mulyadi Djer", Sukman Putra* and Hasri Samion* \\ "Department of Child Health, University of Indonesia, Jakarta, Indonesia \\ "Institute Jantung Negara, Kuala Lumpur, Malaysia
}

Background: Recently transcatheter closure of ventricular septal defect (VSD) is more popular compared to surgery because of shorter hospitalisation, eliminated the need for thoracotomy and better cosmetic results without scar.

Objective: To evaluate the efficacy and safety of transcatheter closure of VSD.

Methods: A case series of patients who underwent transcatheter closure of VSD in the Dr Cipto Mangunkusumo Hospital, Jakarta, Indonesia.

Results: In our hospital since February 2002 transcatheter closure of VSD has been done in 43 patients. Amongst perimembranous VSD, 38 were closed using membranous VSD occluder and 3 were closed using ADO II, the remaining 2 were muscular VSD that was closed using Amplatzer muscular VSD occluder and Amplatzer septal occluder, respectively. There were 24 male and 19 female. The age ranged from 5 - 46 years (median 6 years) and the median of body weight was 19 (range 8 - 68) kg. The size of VSD was 3.5 (2.0 - I I.0) mm. Systolic PA pressure was 108 (87 - I64) mmHg and flow ratio was I.3 (0.6 - 5.0 ). The size of devise used was 5 (4-12) mm. Fluoroscopy time was 42 (I0 - 159) minute and procedure time was 147 (38 - 305$)$ minute. Small residual shunt was observed in I patient following VSD closure using ADO II. All patients were discharged the following day after procedure. Patients were followed-up periodically in the clinic with echo-cardiography to monitor the presence of residual shunts or complications. During procedure and follow-up no significant complications occurred and none of the patients had complete AV block.

Conclusion: Based on our experience we conclude that percutaneous transcatheter closure of VSD is effective and save.

\title{
Abstract no: 887 \\ Improved outcomes after percutaneous ultrasound-guided stenting of the fetal atrial septum in left atrial hypertensive cardiac lesions
}

\author{
Edgar Jaeggi", Greg Ryan\#, Michael Seed*, Glen Van Arsdell* and Rajiv Chaturvedi* \\ "The Hospital for Sick Children, University of Toronto, Canada \\ \#Mount Sinai Hospital, University of Toronto, Canada
}

Background: Hypoplastic left heart syndrome with a highly restrictive or intact atrial septum (HLHS-RAS) has a very high mortality. Fetal left atrial (LA) hypertension results in abnormal lung development with lymphangiectasia and severe pulmonary vascular disease. We report our experience of in-utero percutaneous ultrasound-guided stenting of the fetal atrial septum to decompress the LA.

Methods: Retrospective review of fetuses with HLHS-RAS or a variant that underwent active peri-natal management since 2000 at our tertiary care centres.

Results: Ten fetuses were identified. Of 6 cases without pre-natal intervention, 2 died in utero (33, 29 weeks) from progressive hydrops. The 4 fetal survivors required the urgent creation of an atrial communication immediately after birth but died subsequently ( 5 - 54 days). Percutaneous ultrasound-guided catheter interventions were performed in 4 more recent fetuses at 28 - 36 weeks. A $20 \mathrm{~cm}$ non-bevelled I $8 \mathrm{G}$ needle was advanced via the maternal uterus, amniotic fluid, the fetal chest and right atrium across the muscularised thickened inter-atrial septum-to-stent the atrial septum with a coronary stent expandable to about $3.5 \mathrm{~mm}$. Elevated LA pressure, dilatation and Doppler flow pattern of the pulmonary veins, and MRI-estimated lung perfusion all immediately improved after the procedure. 3 of the 4 stented fetuses were born by vaginal delivery. Atrial septectomy and additional surgical procedures were performed within 72 hours of birth. Intra-operative lung biopsy demonstrated muscularised pulmonary veins and lymphangiectasia in all 4 . 2/4 fetuses developed severe or moderate stent stenosis in utero and both died after birth from pulmonary hypertension and sepsis, respectively. The other 2 remain alive $>12$ months of life, a significant improvement in survival compared with non-stented cases $(p=0.03)$.

Conclusion: Fetal LA decompression by atrial septal stenting may decrease lung injury and has the potential to improve survival in HLHS-RAS.

\section{Abstract no: 925 \\ Successful palliative balloon dilation of native coarctation of the aorta in a pre-term very low birth weight infant}

\section{Chanatip Luevisadpaibul" and Anant Khositseth"}

"Faculty of Medicine, Srinak, Bangkok, Thailand

\#Faculty of Medicine, Ramath

Balloon dilation of coarctation of aorta may provide adequate palliation in pre-term infants, by relieving symptoms and allowing growth until definitive surgical repair can be performed. We report successful balloon angioplasty in pre-term very low birth weight (VLBW) infant with coarctation of the aorta.

Case report: The patient was a 30-week gestation pre-term female infant with birth weight of I 050 grams. She had respiratory distress syndrome and required a mechanical ventilator. The echocardiogram reviewed moderate size of ventricular septal defect and patent ductus arteriosus at I 3 days. The aortic arch couldn't be identified due to poor echo window. She was treated with a diuretic. At 1.5 months, she developed hypertension with 5 mmHg. Blood pressure differential in upper and lower extremities. The echocardiogram reviewed coarctation diameter $1.6 \mathrm{~mm}$ with a maximum pressure gradient of (PG) $60 \mathrm{mmHg}$ and a mean 
PG $17 \mathrm{mmHg}$. The balloon dilation was done at 2 months of age with body weight 1000 grams via 4 Fr. sheath in the right femoral artery. The aortogram reviewed hypoplastic transverse arch $3.2 \mathrm{~mm}$, aortic isthmus $2.2 \mathrm{~mm}$, coarctation $1.8 \mathrm{~mm}$ and descending aorta $4.3 \mathrm{~mm}$. Balloon dilation was done by coronary balloon (Hirye) $4 \times 20 \mathrm{~mm}$ with 2 attempts. The peak-to-peak PG across coarctation after dilation was reduced from $70-12 \mathrm{mmHg}$. She was successfully extubated after 2 days of intervention. The echocardiogram reviewed residual coarctation $2.5 \mathrm{~mm}$ with mean PG $9 \mathrm{mmHg}$ after 12 days of intervention. 2 weeks later, the oxygen was weaned off. She was discharged from hospital 3 months after intervention with a body weight 2090 grams.

Conclusion: We succeeded in palliative balloon dilation of coarctation of the aorta in pre-term VLBW infant.

\title{
Abstract no: 926
}

Routine ultrasound-guided venous access for electro-physiology procedures reduces inadvertent arterial puncture and the risk of arterio-venous fistula formation

\author{
Trinette Steenhuis, Antigoni Deri, Michael Blackburn and Dominic Hares \\ Yorkshire Heart Centre, Paediatric Cardiology, Leeds General Infirmary, United Kingdom
}

Background: Electro-Physiology (EP) studies are commonly performed in children and require the insertion of multiple electrodes into the heart. This requires multiple points of venous access, including femoral, subclavian or brachial venous access. There is evidence to suggest that percutaneous femoral venous access carries a risk of inadvertent arterial puncture (IAP) with development of arterio-venous (AV) fistulae. In the United Kingdom the National Institute of Clinical Excellence (NICE) guidance suggests that ultrasound guidance should be used for central venous access.

Objective: To assess whether the routine use of a vascular ultrasound machine (SONOSITE M-TURBO) reduces the risk of arterial puncture and thereby the causation of AV fistulae.

Methods: We utilised a departmental database (Oscar 4D) to perform a retrospective review of the procedural notes on patients who underwent EP studies in our institution from June 2008 - June 2011 . Our database contains information on access methods and complications.

Results: 122 EP studies (87 radio frequency ablations) were performed in 108 children under the age of 16 in the time period reviewed, with between 3 and 5 sites of venous access per study. Successful femoral venous puncture was performed in $100 \%$ of patients successful brachial venous puncture was performed in $97.5 \%$ ( 1 19/122) of patients under echo guidance, with IAP reported in none. There were no reported cases of AV fistulae in over 500 episodes of access, with up to 3 years of post-procedural clinical outpatient review.

Conclusion: Our experience shows that ultrasound guided venous access is safe and achievable for children undergoing EP studies, with a reduction on the expected risk of IAP from published data ${ }^{(1)}$ and no episodes of post-procedural AV fistulae. We recommend the routine use of ultrasound to reduce these potentially significant complications.

Reference: I. A. Pikwer, et al. Scand J Urol Nephrol. Nov 10, 2011.

\author{
Abstract no: 964 \\ Results of treatment of fetal tachycardia in 80 subjects in Sweden \\ Britt-Marie Ekman-Joelsson*, Mats Mellander", Linda Legnefeldt" and Sven-Erik Sonesson" \\ "The Queen Silvia Children's Hospital, Sahlgrenska University, Gothenburg, Sweden \\ \#Astrid Lindgren Children's Hospital, Karolinska University Hospital, Stockholm, Sweden
}

Background: Fetal tachycardia represents a risk for morbidity and mortality and there is no consensus concerning medical treatment.

Aim: To evaluate the treatment of fetal tachycardia with emphasis on choice of first-line agent.

Materials and methods: 80//28 referrals with fetal tachycardia received trans-placental anti-arrhythmic treatment and were reviewed.

Results: Fifty one fetuses were diagnosed with atrioventricular re-entry tachycardia (AVRT); 23 had atrial flutter (AF); 5 had permanent junctional reciprocating tachycardia (PJRT); I junctional ectopic tachycardia (JET); and I atrial ectopic tachycardia (AET). Choice of treatment and results is described in an attached table. Conclusions: In this small series sotalol was more effective than digoxin in terminating tachycardia without hydrops. In hydropic fetuses neither sotalol nor digoxin was effective in terminating the tachycardia as first-line agent.

\section{Abstract no: 993 \\ Transcatheter closure of right duct in an infant with isolated right subclavian artery from main pulmonary artery with pulmonary and subclavian steal syndrome: An extremely rare case report}

\author{
Ratthapon Wongwandee, Boonchu Sirichongkolthong and Utairat Chaumrattanakul \\ Faculty of Medicine, Thammasat University, Bangkok, Thailand
}

Background: Isolated subclavian artery without associated congenital cardiac defect is an extremely rare condition. This anomaly can cause pulmonary and subclavian steal with perfusion of the arm and lung via the vertebra-basilar system. Reimplantation of subclavian artery into the ascending aorta is considered a conventional management. However, transcatheter embolisation may be a proper alternative for a small infant with this condition.

Methods and results: We report an infant girl diagnosed with 22q l 1.2 deletion syndrome who presented with continuous murmur, heart failure and poor perfusion of right arm. The MDCT angiography demonstrated isolated right subclavian artery from main pulmonary artery via right duct with retrograde flow of right vertebral artery. Moreover, collateral branches of prominent right intercostal arteries supplied right subclavian artery. The right duct was completely occluded with vascular plug II to stop pulmonary over-circulation. The angiogram showed that subclavian steal phenomenon was diminished due to duct closure. Right subclavian artery was supplied by right vertebral artery and right intercostal arteries. Consequently, perfusion of right arm and heart failure clinically improved.

Conclusions: Transcatheter occlusion of isolated right subclavian artery from main pulmonary artery with steal phenomenon is effective and alternative treatment for small infant. 


\author{
Abstract no: 994 \\ Elongated patent ductus arteriosus as a risk factor for transcatheter device closure \\ Kolja Becker, Jana Schlangen, Hans-Heiner Kramer and Gunther Fischer \\ I Department of Congenital Heart Disease and Paediatric Cardiology, University Hospital of Kiel, Germany
}

Background: Only a few case reports exist about spontaneous spasm of patent ductus arteriosus (PDA). While PDA spasm may lead to complications of transcatheter PDA closure it still remains unclear if there are common anatomic findings in this group of patients.

Methods: We reviewed aortic angiographies, clinical findings and complications of transcatheter PDA closure performed in our institution since 1994. Angiographic PDA appearances were classified according to Krichenko, et al. (1989).

Results: PDA closure was performed with different devices in $40 \mathrm{I}$ pediatric patients. In all cases an aortic angiogram was obtained before the PDA was passed with the catheter. The majority of patients (57.6\%) had a conical PDA shape (Krichenko Type A), in $2.7 \%$ it was Type B, in $8.5 \%$ Type C and in $7.2 \%$ Type D. In 23.9\% we saw an elongated, sometimes bizarre PDA configuration (Type E). Although it had not been passed by the catheter, the PDA temporarily constricted and closed completely just before or at the beginning of the procedure in 6 patients (I.5\%), all had a Type E PDA ( $p=0.000$ I). An embolisation of the implanted device occurred in 5 patients (1.2\%). Three of 5 embolisations occurred in Type E PDA (relative risk: 4.7, $p=0.09$ ).

Conclusion: Spontaneous spasm of the PDA is a rare phenomenon and it only occurred in patients with a Krichenko Type E PDA. Transcatheter PDA closure in these patients should be carried out with care as it might bear a higher risk of device embolisation.

\author{
Abstract no: 995 \\ Hybrid management of a pre-natal diagnosed pulmonary artery to left atrium communication \\ Nestor Sandoval, Juan Araque, Gino Bresciani, Carlos Obando, Alberto Garcia and Miguel Ronderos \\ Fundacion Cardioinfantil. Bogota, Columbia
}

Background/hypothesis: Symptoms of a Pulmonary Artery (PA) to left Atrium (LA) fistula appear at different moments of childhood depending on the severity of the shunt. In newborns, central cyanosis and severe heart failure are signs that emergency management has to be done. Open heart surgery is the gold standard on the management of PA to LA fistula but with the arrival of new technology, transcatheter interventions are becoming the elective choice in some cases.

Materials and methods: We report a case of a new born with a pre-natal diagnosis of RPA to LA communication managed with a hybrid technique.

Results: A female new born with a pre-natal diagnosis of pulmonary aneurism in the last trimester. After Caesarean section, due to severe heart failure, central cyanosis and hypoxemia, urgent catheterisation was performed and a right pulmonary artery to left atrium fistula with a big pulmonary cavity found. The infant had emergency surgery. Intra-operative findings included type III AP to LA fistula and 4mm PDA. On extracorporeal circulation without aortic clamping the fistula and the PDA were ligated. Because of a persistent image of an upper right pulmonary lobe cavity, catheterisation was made and a $6 \mathrm{~mm}$ plug was implanted in the efferent branch of the fistula making a successful embolisation. The patient was discharged a few days later with a normal development seen in later controls. The result of the hybrid management of this rare entity was successful.

Conclusions: Pre-natal diagnosis is important for planning and performing emergency operations and, under the presence of anomalies in the pulmonary artery, a fistula between the AP to LA must be suspected. Team work for a hybrid procedure is a useful tool to manage this rare entity.

\title{
Abstract no: 1012 \\ Fetal and post-natal outcome of congenital heart disease diagnosed in utero: A single centre experience
}

Pallavi Mahindrakar, Shweta Nathani and Nageswararao Koneti

Care Hospitals, Hyderabad, India

Background: Fetal echocardiogram (FE) is an established procedure to diagnose and prognosticate fetal heart disease. However fetal and postnatal outcomes vary depending on the social, financial and cultural factors. We report our experience of FE and their outcome.

Materials and methods: 1390 abnormal fetal echocardiograms were performed from January 2004 - June 2012 in our centre. 430 (3l\%) fetuses had significant finding in FE. The maternal risk factors, indications, diagnosis and outcome of the fetal heart disease were analysed.

Results: The mean maternal and fetal age was $25.9 \pm 4.1$ years and $25.6 \pm 4.6$ weeks respectively. The indications for FE were: high risk pregnancy (45\%); suspected CHD (22\%); previous sibling with CHD (18\%); arrhythmia (7\%); and intracardiac echogenic focus (8\%). Among abnormal FE in 374 subjects CHD contributes 83.4\%; arrhythmias 13.6\%; and cardiac tumours 2.9\%. Heterotaxy syndrome was the most common diagnosis (20\%), followed by HLHS (I I.6\%), while isolated VSD 9\%, DORV, d-TGA, single ventricle and ToF contributed 6-7\% each. Other defects contributed to $21.4 \%$. One fetus with severe Aortic stenosis underwent balloon valvotomy at 24 weeks. 208 women opted for medical termination of pregnancy, a few even beyond the legal age limit. Fetal SVT was seen in II of which 10 were successfully controlled by trans-placental pharmacotherapy. One fetus with Sinus bradycardia was diagnosed as LQTS after birth. 20 babies had emergency intervention after birth, 5 babies underwent definitive cardiac surgery as a single stage. The remaining babies were on medical follow-up.

Conclusions: Late referral fetal echocardiogram is commonly seen in our geographical location. Good outcome is predicted in a subset of CHD with definitive treatment, arrhythmias and tumours. Late terminations beyond the legal limit are noticed in complex fetal CHD. 


\section{Abstract no: 1013 \\ Changes in health-related quality of life over 3 years following transcatheter pulmonic valve implantation: Results of a controlled international multi-centre trial}

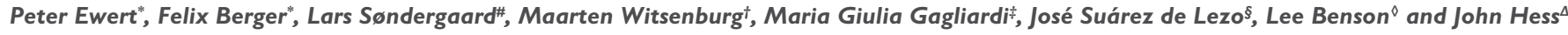
"German Heart Institute Berlin, Berlin, Germany

"Rigshospitalet Copenhagen, Copenhagen, Denmark

tErasmus Medical Centre, Rotterdam, The Netherlands

Hospital for Children, Gesa, Rome, Italy

\$Hospital Reina, Sofia, Cordoba, Spain

"Hospital for Sick Children, Toronto, Canada

German Heart Institute Munich, Munich, Germany

Introduction: Transcatheter pulmonic valve (TPV) implantation has been shown to be an effective treatment for patients with right ventricular conduit failure. In addition to improvements in ventricular function the overall health-related quality of life (QOL) is an important measure of patient satisfaction. The aim of this study is to evaluate long term clinical performance and whether TPV leads to QOL improvement.

Methods: TPV was implanted in 63 patients between October 2007 - April 2009 at 7 centres throughout Europe and Canada. Procedural outcomes, haemodynamic effects and quality of life (EQ-5D) measures were evaluated during pre-implant, discharge, 6 month and annual visits.

Results: 42 patients (age $\geq 15$ years) who completed the EQ-5D assessments pre- and post-implant were included for analysis: 30 were male; mean age 26 I I years (range 15 - 59). The underlying congenital heart diseases included Tetralogy of Fallot (45\%) and truncus arteriosus (17\%). $83 \%$ of conduits were homografts. Compared to pre-implant (8I\%) patients in NYHA class I/II increased at I year (93\%) and sustained through 3 years. Mean pressure gradient preimplant was $37 \pm 12$ and I year post-implant $17 \pm 6 \mathrm{mmHg}$ which remained similar through 3 years. None of the patients experienced moderate/severe pulmonary regurgitation at discharge and throughout 3 year follow-up. EQ-5D index and health state significantly improved at 6 months and was stable through 3 annual visits $(p<0.05)$. Pain/discomfort and anxiety/depression components of the EQ-5D index improved significantly at 6 months and I year (anxiety/depression through 3 years, $p<0.05)$.

\begin{tabular}{|c|c|c|c|c|c|}
\hline & $\begin{array}{l}\text { Pre-implant } \\
\qquad(\mathrm{n}=42)\end{array}$ & $6 \begin{array}{c}\text { months } \\
(\mathrm{n}=39)\end{array}$ & $\begin{array}{l}\text { I year } \\
(n=38)\end{array}$ & $\begin{array}{l}2 \text { years } \\
(n=35)\end{array}$ & $\begin{array}{l}3 \text { years } \\
(n=34)\end{array}$ \\
\hline EQ-5D index & 0.94 & 0.97 & 0.97 & 0.97 & 0.97 \\
\hline State of health & 74.1 & 83.2 & 85.6 & 83.0 & 84.7 \\
\hline
\end{tabular}

Conclusion: In addition to the positive haemodynamic effects, TPV leads to QOL improvement. Whether these results support indication of TPV at an earlier stage of valve dysfunction needs to be discussed.

\section{Abstract no: 1018 \\ Successful percutaneous removal of a fractured stent fragment using a novel stretching method \\ Krzysztof Michalak, Paweł Dryżek, Tomasz Moszura and Jadwiga Moll \\ Department of Cardiology, Polish Mother's Memorial Hospital, Research Institute, Lodz, Poland}

A 9 month old infant $(5800 \mathrm{~g}$ ) with hypoplastic left heart syndrome after Norwood procedure and percutaneous balloon plasty with stent implantation into the aortic arch qualified for catheterisation because of stent fracture and dislocation of its distal portion observed on routine X-ray and recoarctation observed on echocardiography. We confirmed that the small distal part, a simple cell of an implanted stent (with only I circuit wire) was fractured and positioned perpendicular to the long axis of the stent. Its fractured part was connected with the main part of the stent by only a single stitch. We decided to redilate the main part of the stent with the distal fragment to stabilise both parts on the aortic wall, but during the procedure the stent fractured completely and the distal part migrated to the descending aorta. After shifting it back and redilatation of the distal fragment, we observed that the dislocated part of the stent had not been adequately fixed, and still tended to change position in the direction to the descending aorta.

The fractured part of the stent was stabilized using 2 forceps. Next a coronary wire was introduced and inserted into the separated round part of the stent and caught with an Amplatz GoosNeck in such a way that the coronary wire made a loop, catching the fractured stent fragment from a femoral and jugular approach. The circular part of the stent changed during stretching in linear shape which allowed us to remove the second loop and pull out the fractured part of the stent via the $6 \mathrm{~F}$ sheath. Next, we redilated the main part of the stent in the aortic arch.

There was no general or local complications with no pressure gradient through the aortic arch and descending aorta and no signs of damage to the aortic wall.

\section{Abstract no: 1054}

Rhythm and conduction abnormalities after transcatheter closure of VSDs: A single centre experience

Sevcan Erdem, Nazan Ozbarlas, Abdi Bozkurt, Alev Kiziltas and Osman Kucukosmanoglu

Faculty of Medicine, Cukurova University, Adana, Turkey

Background: We describe our experience focusing on the acute and mid-term disturbances of conduction and arrhythmias after transcatheter VSD closure, and review their therapy and follow-up.

Material and methods: The medical records of consecutive 45 patients undergoing transcatheter closure of VSD were retrospectively assessed from August 2007 - July 20 I2. All ECG and 24 hours ECG-Holter monitoring records were analysed for disturbances of conduction and arrhythmias. 
Results: Ages at implantation of patients ranges between 15 months and 58 years (mean: 10.8 years, median: 8 years). Mean body weight was $30.2 \mathrm{~kg}$ (range: 8.7 - 78kg, median: $24.5 \mathrm{~kg}$ ). The diameters of VSDs were between 3.5 and $8.5 \mathrm{~mm}$ by echocardiography (mean: $5.6 \mathrm{~mm}$ ). All defects were occluded with Amplatzer devices. Amplatzer peri-membranous VSD occluders were used for 14 patients (mean size of device: $6.4 \mathrm{~mm}$, range: 4 - $9 \mathrm{~mm}$ ), muscular VSD occluder for 26 patients (mean size of device: $6.9 \mathrm{~mm}$, range: $4-10 \mathrm{~mm}$ ) and duct occluder for 5 patients ( 3 of them 6/4; 2 of them 8/6). Mean device diameter to defect diameter ratio was $1.2+0.13$ (range: 1 - 1.4).

The mean duration of follow-up period was 25.7+ 14.2 months (range: I - 49 months). 2/29 patients with peri-membranous VSDs developed cAVB within 2 and 6 days from the closure. Insignificant rhythm disturbances were developed in 26.7\% (I2/45) of patients after transcatheter closure of VSDs.

Conclusion: During to the early and late occurrence of CAVB, careful monitoring of rhythm and atrioventricular conduction is essential during the follow-up period. However, Holter monitoring should be performed before the procedure to understand the insignificant rhythm disturbances which were related the procedure.

\section{Abstract no: 1059}

\section{Transcatheter closure of PDA using Amplatzer duct occluders: A single centre experience}

\section{Nazan Ozbarlas, Sevcan Erdem, Osman Kucukosmanoglu, Alev Kiziltas and Abdi Bozkurt}

Faculty of Medicine, Cukurova University, Adana, Turkey

Background: In this report we review initial and 4 year results following transcatheter occlusion of patent ductus arteriosus (PDA) using Amplatzer duct occluders (ADO).

Method and materials: From November 2004 - May 20 I2, 9 I patients, ages 3.5 months - 66 years, underwent transcatheter closure using the ADO and ADO II. The mean pulmonary artery pressure was $25.4 \pm 8.6 \mathrm{mmHg}$ (median $23 \mathrm{mmHg}, 12-60 \mathrm{mmHg}$ ) and 5 I of them $>25 \mathrm{mmHg}$.

Results: The mean PDA diameter (at the pulmonary end) was $3.2 \pm 1.2 \mathrm{~mm}$ (range $1.6-7.5 \mathrm{~mm}$ ), the PDA length $6.9 \pm 3 \mathrm{~mm}$ (median 6 , range $3-16 \mathrm{~mm}$ ) and the mean ampulla diameter (at the aortic end) $10 \pm 3.9 \mathrm{~mm}$ (median 9, range $4-23 \mathrm{~mm}$ ). For PDA closure I 30 ADO, 4 I ADO II and I4 ADO AS were used. Except for occlusion of the femoral artery in one patient, there were no complications in the early stages. Complete closure was observed in 56/57 (98.2\%) ADO patients, 19/20 (95\%) in ADO II patients and I4/I 4 (I00\%) in ADO I| AS patients at end of the 6th month after closure.

In conclusion: All ADO types can be used safely and effectively in the treatment of PDA. ADO II and ADO II AS have many advantages in small infants and short ductus. These devices can be deployed completely in the ductal body without any obstruction because of their special configuration. They have smaller sheath sizes and flexible, user-friendly delivery system.

\section{Abstract no: 1066 \\ Extra-cardiac findings in fetal laterality disturbance: The unusual suspects}

\section{Caroline Jones, John Simpson, Owen Miller and Gurleen Sharland}

Evelina Children's Hospital, London, United Kingdom

Background: In pre-natal congenital heart disease, the group with laterality disturbance are most likely to have associated extra cardiac abnormality (ECA). ECAs are multi-system, vary in severity and may be difficult to detect pre-natally. We report a large series of pre-natal cases with laterality disturbance and describe their associated extra cardiac malformations.

Materials and methods: We performed a retrospective search of our fetal cardiology database from 1985 - 2010 for cases of laterality disturbance. Post-mortem (PM) and post-natal notes were reviewed to document ECA.

Results: A total of 255 cases of laterality disturbance were identified. Three cases were excluded as outcome data was not available. Of the 252 cases, 120 resulted in termination of pregnancy (TOP), 16 intra-uterine deaths occurred and 54 patients were still alive. PM reports were available in 86 (72\%) cases following TOP. Common findings were bowel malrotation (84 cases, 33\%), polysplenia (62 cases, 25\%) and asplenia (60 cases, 24\%). An additional 43 patients had other ECAs. Of those live born, 23 (20\%) patients underwent 29 non-cardiac surgical procedures including 17 Ladd procedures, 2 Kasai procedures for biliary atresia and 2 congenital diaphragmatic hernia $(\mathrm{CDH})$ repairs. Four patients died as a direct result of ECA. Three had significant respiratory compromise as a result of $\mathrm{CDH}$ or major airway abnormality. One patient died following surgery for volvulus.

Conclusions: Intra-cardiac pathology is the pre-dominant cause of death in the fetus diagnosed with laterality disturbance. The ECAs influence survival in a minority of cases, though they may have a significant impact on the quality of life of survivors. Particular attention should be given to discussing potential ECA when laterality disturbance is diagnosed.

\section{Abstract no: 1068}

\section{Radiofrequency ablation of atriofascicular pathways in children and adolescents}

Roman Gebauer", Christian Paech", Jan Janousek", and Ingo Daehnert"

"Paediatric Cardiology, Heart Center Leipzig, University of Leipzig, Germany

\#Children's Heart Centre, University Hospital Motol, Prague, Czech Republic

Objective: Atriofascicular (Mahaim) pathways (MP) are a rare reason for supraventricular tachycardia in children and adolencents. We analysed results of RF ablation and further clinical course of patients (pts) with AV re-entrant tachycardia due to MP.

Methods: Data from 22 consecutive pts. from 2 centers aged 7.6 - 19.6 (median I4. I) years, who underwent RF ablation of a MP between I998 - 201 I (followup: median 18.7, range $0.2-62.4$ months) were analysed.

Results: MP was located at right lateral (IOpts) or posterolateral (I pts) tricuspid annulus. Mapping was based on the finding of a specific Mahaim potential in 15/22pts and/or on intermittent catheter-induced mechanical conduction block in the MP in 10/22pts. Acute ablation success rate was 100\%. Of the 22 pts with initially successful ablation, 5 pts had arrhythmia recurrence and 4 of them were successfully re-ablated carrying a total long-term ablation success rate of 
95\% (21/22 pts). The remaining pts is on antiarrhythmic medication. In 6/22pts additional arrhythmogenic substrates were found: WPW syndrome in 2pts, concealed atrioventricular pathway in 2pts and AVNRT in 2pts. All of these substrates were successfully ablated. Mean fluoroscopy time was I8.2 (range 5.9 - 64.3) minutes. There were 2 minor procedure-related complications, no major complications were observed.

Conclusions: Mapping of MP may be hampered by frequent mechanical conduction block. Ablation therapy is effective and incidence of MP recovery approaches figures reported for conventional right free wall pathways.

\title{
Abstract no: 1077 \\ Usefulness of long term administration of heparin in the recanalisation of femoral arteries in young children after percutaneous procedures
}

\author{
Jacek Kusa*, Leslaw Szydlowski*, Agnieszka Skierska*, Zbigniew Olczak\# and Ewa Nowakowska* \\ "Department of Pediatric Cardiology, Medical University of Silesia, Katowice, Poland \\ \#Department of Pediatric Radiology, Medical University of Silesia, Katowice, Poland
}

\begin{abstract}
Introduction: As a result of interventional and diagnostic procedures performed in young children due to congenital heart defects some vascular complications may present. The obstruction of the femoral artery is the most often problem.

Material and methods: From March 2010 - July 2012 we performed 176 heart catheterisations, including 95 children $<3$ years of age who underwent catheterisation through the femoral artery access. The presence of arterial pulse on lower extremities was examined by palpation in all patients. In case of doubt or lack of pulse, ultrasound vascular flow was performed. In 6 patients, aged $20.5 \pm 9.7$ months (0.2 - 30) weighing 9.2 $\pm 4.0 \mathrm{~kg}$ (3.3 - 15 ), there was no flow through the punctured artery. The consulting vascular surgeon in all cases found no need for surgical treatment.

Results: All 6 patients received continuous infusion of heparin. When the absence of flow was still observed we started the treatment with low molecular weight heparin. As a result, after 19.7 10.5 days (6 - 40) of the intake of heparin, in 5 patients the complete vessel patency returned. We have no data about last patient as he was transferred to another department.

Conclusions: Heart catheterisation through femoral artery access in young children is associated with an increased probability of closure or obstruction. Long term administration of heparin is associated, in most cases, with total arterial recanalisation.
\end{abstract}

\section{Abstract no: 1086 heart disease

\author{
Gesa Wiegand", Ludger Sieverding*, Wolfgang Bocksch\# and Michael Hofbeck \\ "University Children's Hospital, Tübingen, Baden-Württemberg, Germany \\ \#University Hospital of Tübingen, Baden-Württemberg, Germany
}

Percutaneous closure of shunt fistulas with the Amplatzer vascular plug IV in patients with congenital

Background: Vascular plugs allow the interventional closure of large-to-medium sized abnormal vessels, but their application is limited by the need for long sheaths or large guiding catheters. We report our experiences with the new Amplatzer Vascular Plug IV (AVP IV). This self expanding spindle-shaped occluder is made of a Nitinol wire mash and it is possible to place it through a 4 to 5 French catheter with a lumen of $0.038^{\prime \prime}$.

Method and material: From October 2009 - June 20I2, I4 AVP IV devices were deployed in 12 patients (age range 3 month to 48.8 years, weight 6.3 - 60.6kg). Results: Nine patients had venovenous or arteriovenous collaterals in functional univentricular hearts; I had pulmonary atresia with ventricular septal defect and major aorto-pulmonary collateral arteries; I had a pulmonary arteriovenous fistula; and I child had a large coronary artery fistula. We used AVP IV devices with diameters of 4-6mm and $8 \mathrm{~mm}$. In all 12 patients the AVP IV was implanted successfully, in 2 patients 2 different vessels were occluded with AVP IV devices and there was no occluder dislocation. In 2//4 vessels it was necessary to place additional devices in the presence of a residual shunt. Complete vessel occlusion was achieved in 7 cases, and in a further 7 patients a residual shunt was present at the end of the procedure while patients were still fully heparinised. There were no complications related to the procedure.

Conclusion: Based on our experience, the AVP IV allows safe and effective occlusion of medium size and large abnormal vessels. It is also well suited for tortuous high flow vessels such as coronary or pulmonary arteriovenous fistulas. In case of suboptimal position it is possible to reposition the occluder with ease. The AVP IV represents a valuable new device in the interventional treatment of complex congenital vessel malformations.

\section{Abstract no: 1092 \\ Pre-natal diagnosis of major congenital heart disease: Combining U.K. Central cardiac audit database and maternity audit to obtain centre-specific detection rates in I 20 I98 screened women}

Helena Gardiner*, \#, Alexander Kovacevic*, Laila van der Heijden*, Joan Larovere* and Rodney Franklin*

"Royal Brompton NHS Foundation Trust, London, United Kingdom

"Imperial College, London, United Kingdom

Background/hypothesis: Fetal cardiac screening standards introduced by NHS Fetal Anomaly Screening programme for English maternity units, 20 I0, have no current baseline data. In contrast, pre-natal diagnosis for infants undergoing transcatheter or surgical treatment is routinely collected and validated by the Central Cardiac Audit Database (CCAD) (www.ccad.org.uk).

Hypothesis: A national system is vital to measure prenatal detection of major congenital heart disease (CHD).

Material and methods: 120198 unselected, pregnant women were screened in 3 maternity units referring to I surgical centre:

A: Co-located with fetal medicine unit; ready access to second opinion; training in major cardiac malformations;

B: Received on-site training and telemedicine support by peri-natal cardiologist; and

C: Supported by local obstetricians with scanning expertise.

We cross-referenced maternity records and CCAD to include cases unreported by obstetric screening 
Results: 388 CHD from 120,198 (3.2/1,000) screened women were documented (Table). Detection by unit (A, B, C): simple TGA (78\%, 50\%, 0\%) and coarctation $(82 \%, 20 \%, 13 \%)$.

\begin{tabular}{|c|c|c|c|c|c|c|c|c|c|c|c|}
\hline & 2000 & 2001 & 2002 & 2003 & 2004 & 2005 & 2006 & 2007 & 2008 & 2009 & 2010 \\
\hline A scans & 4098 & 4132 & 4601 & 4767 & 4822 & 4873 & 4906 & 4926 & 5097 & 5089 & 5094 \\
\hline $\mathrm{CHD}$ & 14 & 16 & 21 & 29 & 22 & 21 & 15 & 17 & 9 & 23 & 22 \\
\hline PD (\%) & $10(71)$ & $13(8 \mid)$ & $15(7 \mid)$ & $24(83)$ & 13(59) & $16(76)$ & 13(87) & $15(88)$ & 7(78) & 19(83) & $16(73)$ \\
\hline B scans & 3880 & 3886 & 3679 & 4015 & 3998 & 4106 & 4260 & & & & \\
\hline $\mathrm{CHD}$ & 16 & 12 & 13 & 13 & 3 & 6 & 9 & & & & \\
\hline PD (\%) & $2(13)$ & $6(50)$ & $4(3 \mid)$ & $6(46)$ & I(33) & $4(67)$ & $8(89)$ & & & & \\
\hline C scans & 3300 & 3298 & 3341 & 3402 & 3409 & 3435 & 3426 & 3957 & 4048 & 4202 & $4|5|$ \\
\hline $\mathrm{CHD}$ & 7 & 12 & 2 & 5 & 10 & 6 & 9 & 9 & 13 & 17 & 17 \\
\hline PD (\%) & $4(57)$ & $6(50)$ & $0(0)$ & $2(40)$ & $5(50)$ & $3(50)$ & $8(89)$ & I(II) & $6(46)$ & $5(29)$ & $7(4 I)$ \\
\hline
\end{tabular}

Conclusions: Combining pre-natal diagnosis (PD) with CCAD produced maternity hospital and lesion-specific detection rates and confirms that continued improvement in $\mathrm{CHD}$ detection depends on integration of health information systems to enable tracking between maternal and infant records.

\section{Abstract no: 1095}

\section{Fetal laterality disturbance: Do atrial appendages matter?}

\section{Caroline Jones, John Simpson, Shane Tibby, Owen Miller and Gurleen Sharland}

Evelina Children's Hospital, London, United Kingdom

Background: Laterality disturbance in the fetus is associated with a wide spectrum of cardiac abnormalities. Many present pre-natally with complex morphology where single ventricle palliation is the only management option after birth. The cumulative effect of associated lesions means many do not achieve Fontan completion long term. We report a large pre-natal series describing their associated malformations and review factors that influence their outcome.

Materials and methods: We performed a retrospective search of our fetal cardiology database from 1985 - 2010 for cases of laterality disturbance. Pre- and post-natal management and long term outcomes were documented.

Results: A total of 255 cases of laterality disturbance were identified. 3 cases were excluded as outcome data was not available. Of the 252 cases, 120 resulted in termination of pregnancy (TOP) and 16 intra-uterine deaths (IUD). Fifty four patients remain alive, 20 with a Fontan circulation, 2 patients have undergone cardiac transplantation. In those receiving active treatment after birth, univentricular physiology, complete heart block, total anomalous pulmonary venous drainage and right heart outlet obstruction were independent risk factors for death or trans-plantation $(p<0.05)$.

TABLE I: Outcome following pre-natal diagnosis of laterality disturbance depending on circulation type and era

\begin{tabular}{|c|c|c|c|c|c|c|c|}
\hline & & Alive (\%) & TOP (\%) & IUD (\%) & NND (\%) & INFD (\%) & Total \\
\hline \multicolumn{8}{|c|}{ Biventricular } \\
\hline \multirow[t]{3}{*}{ Era } & Pre 2000 & $12(22)$ & 27 & 5 & 7 & 4 & 55 \\
\hline & Post 2000 & $13(38)$ & 10 & 4 & 5 & 2 & 34 \\
\hline & Total & $25(28)$ & $37(42)$ & $9(10)$ & $12(13)$ & $6(7)$ & 89 \\
\hline \multicolumn{8}{|c|}{ Univentricular } \\
\hline \multirow[t]{3}{*}{ Era } & Pre 2000 & $14(16)$ & 48 & 4 & 14 & 9 & 89 \\
\hline & Post 2000 & $15(20)$ & 35 & 3 & 8 & 13 & 74 \\
\hline & Total & 29 (18) & $83(5 \mathrm{I})$ & $7(4)$ & $22(13)$ & $22(13)$ & 163 \\
\hline
\end{tabular}

TOP: termination of pregnancy. IUD: intra-uterine death. NND: neonatal death. INFD: infant death.

Conclusions: Prognosis remains poor for those with univentricular physiology with an ongoing high risk of mortality throughout childhood. Survival in this group has not significantly changed across the study period. Detailed pre-natal assessment to identify specific morphological features associated with poor prognosis may help guide fetal counselling. In addition reduced quality of life remains a serious concern for the few survivors. 


\section{Abstract no: II 24 \\ Outcomes of Amplatzer duct occluder II (ADO II) device occlusion of patent ductus arteriosus (PDA): The North West experience}

\section{Atul Kalantre, Arul Narayanan, Arjamand Shauq, Gordon Gladman, lan Peart, Prem Venugopal and Edmund Ladusans}

Alder Hey Children's NHS Foundation Trust, Liverpool, United Kingdom

Background: The Amplatzer Duct Occluder II (ADOII) is a self-expanding nitinol device for closure of patent ductus arteriosus (PDA). This device is designed to produce high rates of occlusion but there has been recent concern as to device deformability and embolisation.

Objective: To evaluate our experience of using the ADO II device and document the closure rate and incidence of complications.

Method: A retrospective analysis of data of patients on whom we attempted closure of PDA using the new device from July 2008 - June 2012 were included ( $n=67$ ). Results:

\begin{tabular}{lccc}
\hline \hline Demographics $(\mathrm{N}=67)$ & Redian & Ductal Morphology \\
\hline Age $(\mathrm{months})$ & 20 & $5-130$ & $\mathrm{~A}=28(42 \%)$ \\
\hline Weight $(\mathrm{Kg})$ & 10 & $5.2-39$ & $\mathrm{~B}=12(18 \%)$ \\
\hline Narrowest ductal diameter $(\mathrm{mm})$ & 2.6 & $1.5-4.9$ & $\mathrm{C}=18(27 \%)$ \\
\hline Length of duct $(\mathrm{mm})$ & 7 & $3.3-17$ & $\mathrm{D}=2(03 \%)$ \\
\hline Diameter of aortic ampulla $(\mathrm{mm})$ & 7.7 & $3-13$ & $\mathrm{E}=7(10 \%)$ \\
\hline Procedure time $(\mathrm{min})$ & 48 & $22-114$ & \\
\hline Fluoroscopy time $(\mathrm{min})$ & 7 & $2.8-23.3$ & \\
\hline
\end{tabular}

"Krichenko classification of PDA morphology

Devices used were 3/4 (I2), 4/4 (30), 5/4 (15), 6/4 (3), 4/6 (3), 5/6 (2) and 6/6 (2). The venous approach was employed in all. Echocardiography was done on $\mathrm{DI}$ and 4 - 6 weeks post implantation. One device was not deployed successfully. Complete angiographic closure occurred in 55, trivial contrast flow in 10 and mild flow in one patient. In 65 patients, echocardiography the next day and on follow up scan at 6 weeks showed no residual shunts. In I patient mild flow was noted which resolved at 4 weeks. 3 devices embolised, I within 6 hours and two the next day. There were no procedure-related complications.

Conclusion: ADO2 is a safe and effective device for PDA occlusion and has a high early closure rate and low embolisation rate.

\section{Abstract no: II33 \\ Improved long term outcome in patients undergoing percutaneous pulmonary valve implantation at a younger age}

\section{Sharon Borik", Rajiv Chaturvedi", Kyong Jin Lee*, Mark K. Friedberg", Brian McCrindle", Andrew Crean", Marc Osten", Eric M. Horlick" and Lee N. Benson",\#}

"Hospital for Sick Children, Labatt Family Heart Centre, Toronto, Canada

\#Toronto General Hospital, Peter Munk Cardiac Centre, Toronto, Canada

Background/hypothesis: Percutaneous pulmonary valve implantation (PPVI) has become increasingly accepted as a safe, less invasive alternative to surgical pulmonary valve replacement for congenital heart disease patients with right ventricular outflow tract dysfunction. Several series have demonstrated favourable short term outcome after PPVI. The aim of this study was to determine if reverse RV remodelling following PPVI is persistent in the long term and whether earlier timing of PPVI may be favourable.

Methods: PPVI patients from the Hospital for Sick Children (HSC) and Toronto General Hospital (TGH) were studied. Cardiac MRI, echocardiography, metabolic exercise testing, and haemodynamics prior to intervention were compared to repeat measures on follow-up, using paired tests and linear regression models assessing changes over time.

Results: Fifty one patients were followed for up to 6.6 years (4.2 \pm 1.9 years) after PPVI, including 35 HSC patients, averaging $14.8 \pm 8.9$ years of age at intervention

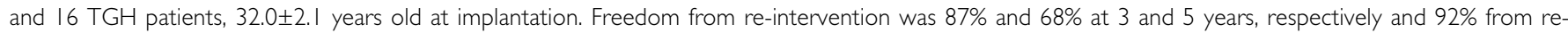
operation at 5 years. Younger age at implantation was associated with an increase of $4.17 \pm 1.29 \% / 10$ years of age in echo LV ejection fraction (LVEF) ( $p=0.00 \mathrm{I}$ ), $0.23 \pm 0.10$ points (on a 3-point scale) per 10 years in qualitative RV function $(p=0.03), 2.68 \pm 0.90 \mathrm{ml} / \mathrm{kg} / \mathrm{min}$ per 10 years in max VO2 ( $p=0.003)$ and a decrease of $0.8 \mathrm{I} \pm 0.19 \mathrm{~cm}$ per decade in RVED ( $p<0.00 \mathrm{I}$ ). Preparing the conduit for implantation (pre-stenting) yielded an increase of $5.32 \pm 1.95 \%$ in $L V E F(p=0.006)$ and $0.63 \pm 0.18$ points in RV function ( $p<0.00 \mathrm{I}$ ) as well as a decrease of $0.80 \pm 0.30 \mathrm{~cm}$ in RVED ( $=0.007)$ and $8.77 \pm 3.39 \mathrm{mmHg}$ in RV systolic pressure ( $p=0.01$ ). Conclusions: This is the largest series to show that PPVI at a younger age has improved long term outcomes. Strategies to preserve right ventricular function should be considered in management planning for this population. 


\section{Abstract no: II54}

\section{Transcatheter closure of persistent ductus arteriosus: Clinical characteristics and outcomes}

\section{Suprohaita Budiyarso, Mulyadi Djer and Sukman Putra}

University of Indonesia, Cipto Mangunkusumo Hospital, Jakarta, Indonesia

Background: Transcatheter closure of persistent ductus arteriosus (PDA), as alternative to PDA ligation, has shorter hospital stays, fewer major complications, and comparable success rates. Studies suggest a success rate of $90 \%$ to $99 \%$ for transcatheter closure of PDA, with the higher success rates being associated with newer devices.

Methods: A descriptive analysis was done in 285 consecutive patients with diagnosis of small to moderate PDA (279 patients) and residual PDA after PDA ligation (6 patients) since January 2002 - July 2012 who underwent transcatheter closure of PDA. Data was collected from medical records and was analysed by descriptive statistic.

Results: From January 2002 - July 2012, 285 patients had transcatheter closure of PDA. The median of age was 3.5 years (40 days - 37 years). 197 patients were female (69.1\%) and median of weight was 12 (3.6-59) $\mathrm{kg}$. Median size of PDA was 3.6 (I - I5.6) mm. Amplatzer ductal occluder I (ADO I) was used in 259 (90.9\%) patients. The median of ADO I device was 6/8 (4/6 - |4/16) mm. Other devices were Gianturco coil (5.6\%), ADO II (I.8\%), pfm nit coil (I.I\%), and Amplatzer septal occluder (0.4\%). The median of flow ratio was I.8 (0.6-14.5), fluoroscopy time was I 5.4 (2.3 - 80 ) minutes, and procedure time was 76 (30 - 198) minutes. Median length of hospital stay was 3 (3 - 4) days. Overall, success rate was $99.3 \%$ and $98.2 \%$ of patients had no major complication. Transient bradycardia occurred in 3 patients and migration of device in 2 patients. Evaluation of echocardiography showed most of patients had complete closure of PDA. Almost all of them discharged I day after procedure.

Conclusions: Transcatheter closure of PDA is safe and effective alternative to surgery. This procedure has high success rate, short length-of-stay in hospital, and minimal complications.

\section{Abstract no: | | 8 | \\ Fetal cardiac disease and fetal lung volume: An in utero MRI investigation \\ Elisabeth Mlczoch", Lisa Schmidt", Maximilian Schmid", Gregor Kaspriant, Daniela Prayert, Ina Michel-Behnke* and Ulrike Salzer-Muhar" \\ "Paediatric Heart Centre, Department of Paediatrics and Adolescent Medicine, Vienna, Austria \\ "Department of Obstetrics and Gynaecology, Division of Obstetrics and Foeto-maternal, Vienna, Austria \\ tDepartment of Radiology, Division of Neuroradiology and Musculoskeletal, Vienna, Austria}

Background: Fetal magnetic resonance imaging (MRI) has become a good non-invasive tool to study fetal lung volumes after 18 GW in vivo. In fetuses with congenital heart disease (CHD) proper lung function is essential for post-natal survival. Pulmonary hypoplasia is associated with a high morbidity and mortality. Ante-natal detection of abnormal pulmonary development may help to optimize pre- and peri-natal management of the fetus at risk. The aim of our study was to investigate fetal lung volume in fetuses with pre-natally diagnosed heart disease.

Material and methods: A cross sectional retrospective study of 105 consecutive singleton pregnancies that underwent MRI was performed. The heart defects were divided into 4 groups and compared within the groups, and also with a normal collective. To compare the lung volumes we calculated $z$ - scores (normal range: Z-score -2 to +2). Our focus of interest was the lung volume of fetuses with heart disease, compared to healthy fetuses and to calculate Z-scores for these fetuses.

Results: Fetuses with CHD have significantly smaller lung volumes compared to healthy fetuses corrected by GA ( $p=0.049$ ). No difference was found within the specific groups. Z-scores: I8/105 fetuses had lung volumes with a Z-score <-2.

Conclusion: Fetuses with CHD show similar lung volume within different types. Our data might indicate that post partum pulmonary symptoms in neonates with congenital heart disease may be contributed more to the lower lung volume than to the cardiac disease itself.

\section{Abstract no: I 84 \\ Primary stent therapy for shunt malfunction in infants}

Nancy Dobrolet*, Robert Hannan*, Redmond Burke*, Michael Lopez* and Evan Zahn\#

"Congenital Heart Institute, Miami Children's Hospital, Miami, Florida, United States of America

${ }^{*}$ Cedars-Sinai Medical Centre, Los Angeles, California, United States of America

Background: Stent therapy (ST) for BT shunt malfunction (SM) is a viable alternative to surgical shunt revision. In our institution, ST is the preferred method of treatment for SM.

Methods: A retrospective analysis of all infants with suspected SM was performed including demographics, catheterisation findings, intervention type, survival to 2nd operation and evidence of bacteraemia.

Results: Seventeen patients presented with SM (s/p Norwood (5), Damus-Kaye-Stansel/shunt (12)) with a median age and weight: 31 days (7 days - 2 years) and $3.2 \mathrm{~kg}(2.6-14.2 \mathrm{~kg})$. Five presented to the lab requiring mechanical cardiopulmonary support. Primary ST was attempted in $15 / 17$ pts with technical success in all 15 pts. One underwent primary angioplasty and I patient arrested during the diagnostic part of the case and underwent emergent surgical revision. Both of these patients survived to 2nd operation. 4/I5 (26\%) stented patients died prior to discharge; 2 arrived at the lab requiring mechanical support, and 2 had preexisting bacteraemia. All remaining ST patients (I I/I5 (73\%)) survived to 2nd operation, resulting in overall survival to 2nd operation of 77\% (I3//7). Etiology of SM: discrete stenosis (12), thrombus (3), or a diffusely small shunt (2). Stenosis at the distal shunt anastomosis occurred in $10 / 12$ patients, 3 of whom also had evidence of thrombus within the shunt. 2 patients with diffusely small shunts underwent successful primary stenting with enlargement of overall shunt diameter, both surviving to 2 nd operation.

Conclusions: A programmatic approach of primary stent therapy for SM in infants has a high technical success rate and acceptable survival to 2nd planned operation. Discrete stenosis most often occurs at the distal anastomosis and may be associated with thrombosis. Pre-existing bacteraemia and/or requirement for mechanical circulatory support prior to ST were associated with high mortality in this small series. 


\title{
Abstract no: I I 87 \\ Fetal supraventricular tachycardia (SVT): Comparison of 2 drug treatment protocols
}

\author{
S. Sridharan", I. Sullivan", V. Tomek", J. Wolfenden*, J. Škovránek", R Yates* and J Marek*,\# \\ "Great Ormond Street Hospital, London, United Kingdom \\ \#University Hospital Motol, Prague, Czech Republic
}

Background: The best treatment for sustained fetal SVT with I:I arterio ventricular relationship is not known. We compared 2 treatment protocols. Methods: I55 consecutive fetuses with supraventricular tachyarrhythmia presented 2000 - 20I2: I27 had SVT with I:I arterio-ventricular conduction; 86 received drug treatment according to I of 2 protocols: first-line maternal intravenous digoxin $(n=52$, centre 2$)$, or maternal oral flecainide $(n=34$, centre $\mid$ ) Treatment success was defined as live birth after conversion to sinus rhythm, or rate reduction by $>15 \%$.

Results: Short ventriculo-atrial (VA) interval occurred in 69 and long VA in 17. Age at treatment start was median 3 I weeks gestation in each centre. Hydrops was present in 30/86 (35\%). Digoxin was successful in 23/28 (82\%) and flecainide in 26/27 (96\%, p0.19) of non-hydropic fetuses, compared to 8/2I (38\%) and $6 / 7$ (86\%, p0.07) respectively when hydrops was present. For short VA SVT, conversion to sinus rhythm and rate control was $31 / 44$ (70\%) and 0/44 for digoxin, and 23/25 (92\%) and I/25 (cumulative 96\%, p0.0I) for flecainide. For long VA SVT, conversion to sinus rhythm and rate control was 4/8 (50\%) and 0/8 for digoxin, and 5/9 (55\%) and 2/9 (cumulative 78\%, p0.3) for flecainide. 2nd line drug treatment was added to digoxin in 19/52 (37\%), and to flecainide in 2/34 (6\%, p0.002). Intra-uterine or neonatal death occurred in $9 / 21$ (43\%, including I termination) hydropic fetuses treated with digoxin compared to $0 / 9$ (p0.03) of those treated with flecainide.

Conclusions: Flecainide was more effective than digoxin in short VA SVT, especially when hydrops was present. Additional treatment was used more often in the digoxin protocol. No adverse fetal outcomes were attributed to flecainide.

\author{
Abstract no: I / 88 \\ Fetal atrial flutter (AFI): Comparison of drug treatment protocols \\ S. Sridharan*, V. Tomek", J. Marek*,\#, J. Wolfenden*, J. Škovránek*, R. Yates* and I. Sullivan* \\ "Great Ormond Street Hospital, London, United Kingdom \\ \#University Hospital Motol, Prague, Czech Republic
}

Background: The best treatment for sustained fetal AFI, defined as regular atrial rate $>200 \mathrm{bpm}$ and faster than the simultaneous ventricular rate, is not known. We compared drug treatment protocols.

Methods: Of 155 consecutive fetuses with supra ventricular arrhythmia, presenting 2000 - 20I2, 28 had AFI. 25 received drug treatment according to one of two drug protocols: digoxin \pm sotalol/other drug $(n=16$, centre 2$)$, or digoxin \pm flecainide ( $n=9$, centre I). Treatment success was defined as conversion to sinus rhythm.

Results: Hydrops was present in 7/16 (44\%) in centre 2, and in I/9 (1 1\%, p 0.18) in centre I. Age at treatment was median 32 weeks gestation in centre 2 and 31.4 weeks gestation in centre I (p 0.96).

In non-hydropic fetuses, sinus rhythm occurred with digoxin monotherapy in 7/I7 (4I\%), with digoxin + flecainide in I/I7 (cumulative $47 \%$ ), and with digoxin + sotalol in $1 / 17$ (cumulative 53\%). In hydropic fetuses, sinus rhythm occurred with digoxin monotherapy in 3/8 (38\%), with digoxin + sotalol in $2 / 8$ (cumulative $63 \%$ ), digoxin + sotalol + amiodarone in I/8 (cumulative 75\%), and with digoxin + propranolol in I/8 (cumulative 88\%). Hydrops was present in I/9 (I I\%) of fetuses with sustained AFI compared to $7 / 16$ (44\%) of those converting to sinus rhythm (p0. I 8 ). Intra-uterine death did not occur (0/8 hydropic fetuses, $95 \%$ Cl 0-0.37). Gestation at delivery was median 38 (range 31-39) weeks gestation.

Conclusions: Surprisingly, hydrops did not reduce the likelihood of conversion to sinus rhythm, raising the possibility that the natural history of fetal AFI may be more important than a drug effect. Hydrops was also well tolerated in utero in comparison to fetal supra ventricular tachycardia with I:I atrio-ventricular relationship, possibly because of later onset of AFI and more favourable atrio-ventricular coupling.

\section{Abstract no: I I96 \\ Transcatheter closure with use of the Cardio-O-Fix Occluders in I53 patients with congenital and structural heart defects}

Jacek Bialkowski, Malgorzata Szkutnik and Roland Fiszer

Medical University of Silesia, SCCS Zabrze, Poland

\footnotetext{
Introduction: Transcatheter closure of different CHD with the use of nitinol wire mesh occluders has been widely accepted as preferred treatment however, the high cost of these devices limits their clinical application in many countries. Few clinical data are available regarding lower-cost products.

Aim: We evaluated the efficacy and safety of Cardio-O-fix occluders (COF) type: patent ductus arteriosus (PDA), atrial septal defect (ASD), and patent foramen ovale (PFO).

Material and method: From September 2009 - I August 2012 a total 153 patients with congenital and structural heart defects (ages 0.5 - 72 years) underwent transcatheter closure with use of different COF occluders: PFO (63); ASD (36); PDA (47); ruptured Sinus of Valsalva aneurysm (SOVA); and coronary artery fistula to right ventricle (CAF) (I). Patients with PFO had cryptogenic stoke in anamnesis and confirmed R-L shunt through PFO. Mean diameter of ASD was $21 \mathrm{~mm}$, PDA 4.I mm, SOVA $10.0 \mathrm{~mm}$ and CAF $6 \mathrm{~mm}$.

Results: All procedures were successful. In I case PFO COF was withdrawn because of unusual position of the device caused by long tunnel (27mm) of PFO, application of ASD COF was efficient in this case. In all cases complete closure of closed undesirable connections were achieved without any complications in follow-up (from I - 35 months). In case of ASD mean diameter of applied devices was 20 ( 10 - 28mm); in case of PFO mostly 25/I8mm devices were used; in case of PDA devices 4/6 till 10/12; in case of SOVA the PDA COF 10/12 - 16/18; and in case of CAF - PDA COF 8/I0mm.

Conclusions: These results suggest that the COF are safe and effective devices for the transcatheter closure of CHD. For confirmation a randomised controlled trial with more patients and longer follow-up is warranted.
} 


\section{Abstract no: 1205 \\ Mid-term follow-up results of transcatheter treatment in patients with unroofed coronary sinus}

\section{Jou-Kou Wang, Shy-Jye Chen, Jui-Yu Hsu, Su-Man Lin, Ming-Tai Lin, Shuenn-Nan Chiu, Chun-An Chen and Mei-Hwan Wu}

National Taiwan University Hospital, Taipei, Taiwan

Objectives: We present the mid-term results of transcatheter treatment of unroofed coronary sinus (CS) using the Amplatzer septal occluder. Background: The unroofed CS is a rare atrial septal defect communicating the left atrium and CS. Surgery has been the mainstay of treatment.

Methods: In a 4.5-year period, 9 patients (5 males) with ages ranging from 26 - 69 years (median 39 years) diagnosed with an unroofed CS but without a persistent left superior vena cava (PLSVC) underwent transcatheter treatment. Computerized tomography (CT) was performed in 8 patients. Transoesophageal echocardiography (TEE) was used to monitor the procedure.

Results: The mean Qp/Qs ratio was $2.4 \pm$ I and mean systolic pulmonary artery pressure was $35 \pm 19 \mathrm{mmHg}$. An Amplatzer septal occluder was deployed in all 9 patients. The device was implanted in the defect in I patient and at the CS ostium in the other 8 patients. The median device size used was $22 \mathrm{~mm}$ ( $16-28 \mathrm{~mm}$ ). The left disc herniated into the CS in the single patient in whom the device was implanted within the defect. All patients were available for the 3-month followup. None had a residual shunt on the 3-month follow-up echocardiography. One patient died of a stroke 4.5 months after the procedure. At mean follow-up $42.6 \pm 18.3$ months, symptomatic improvement was documented in the remaining 8 patients. All 8 patients had an O2 saturation above $96 \%$.

Conclusions: Transcatheter treatment for unroofed CS without PLSVC using Amplatzer septal occluder is safe and feasible.

\section{Abstract no: 1213 \\ Assessment of the ductus arteriosus in fetuses with right ventricular outflow tract obstruction and ventricular septal defect}

\section{Giulia Tuo, Davide Buzzi and Maurizio Marasini}

Cardiology Unit, G. Gaslini Institute and University of Genoa, Genoa, Italy

Objectives: To describe the ductus arteriosus (DA) in fetuses with right ventricular outflow tract obstruction (RVOTO) and anterior misaligned ventricular septal defect (VSD).

Background: Fetuses with RVOTO may develop significant cyanosis after birth. Pre-natal diagnosis may improve the early post-natal management approach. Although pre-natal detection of RVOTO has been already reported, there is lack of information on ductal morphology and ductal blood flow pattern, during pregnancy and after birth.

Patients and methods: The department registry was retrospectively searched for all patients born between January 2006 and June 2012 with a pre-natal diagnosis of RVOTO and VSD. RVOTO was considered severe if pulmonary artery/aortic diameter ratio was $\leq 0.5$ and pulmonary circulation was ductus dependent. Fetuses with pulmonary atresia or absent pulmonary valve syndrome were excluded.

Results: Out of 50 fetuses, 33 had TF (66\%) and 17 (34\%) had Double Outlet Right Ventricle with subaortic VSD. 5 (I0\%) fetuses had severe RVOTO with reverse flow across the DA. Parents opted for termination of pregnancy (TOP) in 2 cases. The other 3 live -born infants underwent modified Blalock-Taussig (mBT) shunt. 33 (66\%) fetuses had mild RVOTO with normal ductal morphology and flow pattern. There were 9 TOP and 2 neonatal deaths for extracardiac reasons. 12 (24\%) more fetuses had mild to moderate RVOTO but very small ductus with changeable flow pattern, spontaneously closed immediately after birth. 2 of them developed severe hypoxic spells respectively at birth and during the Ist month of life and both had mBT shunt.

Conclusion: In less severe degrees of RVOTO, flow across the DA would be reduced and ductus diameter would be decreased even up to prenatal closure. Ductal morphology assessment may be useful for improving management of neonate with pre-natal mild to moderate RVOTO and small DA who may become cyanotic at birth.

\section{Abstract no: | 22 | \\ The Amplatzer duct occluder II additional sizes device for transcatheter patent ductus arteriosus closure: Initial experience}

Elchanan Bruckheimer, Max Godfrey, Tamir Dagan, Gabriel Amir, Georgy Frenkel and Einat Birk

Schneider Children's Medical Centre Israel, Petach Tikva, Israel

Background: Effective and safe PDA transcatheter closure requires a device suited to the specific ductal anatomy which is appropriate for the patient size. The new Amplatzer Duct Occluder II Additional Sizes (ADOIIAS) device is designed with a range of diameters $(3-5 \mathrm{~mm})$ and lengths $(2-6 \mathrm{~mm})$ and small disks to avoid flow disturbance in the aorta and pulmonary artery.

Materials and methods: Population: All patients who underwent attempted closure with an ADOIIAS device. Following aortography the PDA was closed using a 4-5Fr delivery system from the pulmonary or aortic approach with an ADOIIAS of waist $\geq 1.5$ times the narrowest part of the PDA. Aortography to confirm position and leak was performed before and after device release. Echocardiography was performed before the following day.

Results: Between June 20II and August 20I2, 45 consecutive patients (25 female), median age 3.3 years (0.6 - I5.8) and weight I4.5kg (4 - 79), underwent PDA closure with an ADO II AS device. In $42 / 45$ (93.3\%) a device was successfully deployed (38 aortic). In 4I/42 (97.6\%) the PDA was closed echocardiographically the next day. In one case there was a tiny residual leak. In 3 patients the ADO II AS was unstable and an alternative device was implanted. The mean ratio of ADO II AS:PDA diameter was 2.4 ( \pm 0.52$)$ : I. There were no complications.

Conclusions: We present a large initial clinical experience with the new ADO II AS device which is easily implanted from the aortic side and is safe and effective for PDA closure. The variety of sizes affords intuitive suiting of the device to the specific PDA anatomy. 


\author{
Abstract no: 1224 \\ Identifying predictors of mitral valve (MV) tears resulting from percutaneous balloon mitral valvuloplasty \\ (PBMV): A descriptive study
}

\author{
Jonas Bovijn, Anton Doubell and Phillip Herbst \\ Division of Cardiology, Department of Medicine, Tygerberg Academic Hospital, University of Stellenbosch, Bellville, South Africa
}

Background/hypothesis: Despite selection and procedural refinement for PBMV, the rate of catastrophic MR from MV tears remains fixed at $\pm 5 \%$, reflecting our inability to predict this complication. Valve tears not related to catastrophic MR are incompletely studied. The mechanisms underlying $M V$ tears are presumably common to both these scenarios, creating an opportunity to study predictors of MV tears in this cohort.

Materials and methods: Forty five consecutive patients undergoing PBMV (Inoue-technique) with pre and post PBMV digital echocardiographic images available were assessed. Patients with a clearly identifiable tear of the mitral leaflets or apparatus (MVA) were identified. The mechanism and severity of MR pre- and post PBMV was evaluated. We recorded detailed morphological descriptions of each case; including Massachusetts General Hospital score (MGHS), parameters quantifying subvalvular involvement, detailed leaflet edge and commissural assessment and tear location.

Results: Twelve patients (26.7\%) developed clearly identifiable tears of the MVA. Three patients (6.7\%) had catastrophic tears necessitating early surgery. All patients developed moderate or more MR from their tears. The mean MGHS was 9.5 with subvalvular thickening (3.5) and calcification (2.33) contributing most. Tear location: anterior leaflet 6; posterior leaflet 5; subvalvular I. 9/1 I leaflet tears were located in a thin, calcium-free area of the leaflet adjacent to an area of significant calcification. 6/7 tears that occurred lateral (not central) were located to the side of the most severe subvalvular involvement.

Conclusions: Non-catastrophic MVA tears related to PBMV are more common than previously thought. Longitudinal follow-up of this cohort is required to evaluate outcomes in patients who develop non-catastrophic tears post-PBMV. An interesting interplay appears to exist between the morphology at the edge of the MV leaflets, where tears presumably start, and factors that restrict free movement of leaflet segments. This is being assessed in an ongoing study.

\title{
Abstract no: 1229 \\ Fetal bradycardia and sinus node dysfunction
}

Nathalie Jeanne and M. Bravo-Valenzuela

Taubate University, São Paulo, Brazil

Introduction: Sinus node dysfunction is a common condition in elderly patients or those with post-operative congenital heart disease. It is characterized by an abnormal of cardiac impulse formation. In this report I present a rare case of fetal bradycardia and sinus node dysfunction.

Case description: Pregnant 26-year-old patient was referred due to persistent fetal bradycardia after obstetric ultrasonography. The 23 weeks' gestation echocardiogram showed sinus bradycardia (HR 80 - 87bpm) with normal conduction time from the atria to the ventricles ( I:I) and normal anatomy. Follow-up echocardiograms performed at 29, 32, 35 and 38 weeks' gestation showed profound bradycardia (HR 53 - 62 bpm) with no hydropsy or blocked atrial extra systoles. Maternal antibody titers for SS-A/SS-B and screening for QT long syndrome were negative.

Results: The female infant was born by Caesarean section at 39 weeks' gestation and had Apgar scores of $8 / 8$. After birth, the baby developed profound bradycardia (HR 50bpm), poor perfusion, and signs of shock. Atropine and isoproterenol were administered IV to the patient with no significant improvement in HR (55 - 60bpm). The initial ECG showed junctional rhythm at 55bpm, normal QTc (0.42 s), atrial extra systoles, and variable second-degree AV block. A 24-hour ECG and oesophageal ECG allowed the diagnosis of sinus node dysfunction to be confirmed. The echocardiogram demonstrated 2 small ostium secundum atrial septal defects. Possible sinus bradycardia causes were dismissed, thus confirming the above diagnosis. The baby developed oliguria and heart failure at which time the implantation of a pacemaker was indicated. The surgery was successful, and the patient is currently growing and thriving at 24 months of age.

Conclusion: The author reports the importance of fetal echocardiography for the diagnosis and appropriate therapy approach to avoid complications.

\section{Abstract no: 1233 \\ Successful conservative treatment for haemolysis after transcatheter closure of ventricular septal defect (VSD) using Nit-Occlud ${ }^{\circledR}$ Lê VSD occluder}

\author{
Supaporn Roymanee*, Worakan Promphan*, Nakharin Tonklang*, Kanjarut Wongwaitaweewong* and Worakan Promphan" \\ "Prince of Songkla University, Hat Yai, Thailand \\ ${ }^{*}$ Queen Sirikit National Institute of Child Health, College of Medicine, Rangsti University, Bangkok, Thailand
}

Background: We report a haemolysis-associated transcatheter VSD closure using Nit-Occlud ${ }^{\circledR}$ Lê VSD occluder (LVO) that led to transient renal failure and recovery by conservative treatment.

Case: A I-year-old, $8 \mathrm{~kg}$ boy with an underlying disease of Cri du Chat syndrome underwent transcatheter VSD closure. An LV angiogram revealed a large perimembranous VSD with a tricuspid pouch ( I I mm). Initially, a Cocoon Duct Occluder (CDO) (I2//4) was placed resulting in mild aortic regurgitation. A CDO (6/8) was placed instead but resulted in a residual moderate shunt. When a LVO coil (I2/6) was used instead of the CDO it showed another RV exit $(5 \mathrm{~mm})$. So we placed a second LVO coil (8/6) into that exit. A repeat LV angiogram revealed a trivial residual shunt. Unfortunately the LVO coil (I $2 / 6)$ got stuck during deployment, but it was retrieved and another LVO coil (10/6) was placed instead. After the 4-hour procedure, the result was a mild residual shunt.

Results: Unfortunately, he didn't gain full consciousness when weaned off anaesthesia. An emergency craniotomy and clot removal was done after a CT brain scan showed a large subdural haematoma. At ICU his dark brown urine suggested haemolysis, which was confirmed by urinalysis. Due to the complicated procedure, we avoided unnecessary intervention.

Our strategy for the haemolysis was aspirin cessation, IV hydration, alkalinisation of urine and to maintain the haemoglobin $>$ I 2gm\%. His baseline BUN/creatinine was 14.1/0.32 mg\%, which increased slowly in the first I0 days then rose rapidly to $31.2 / 0.57 \mathrm{mg} \%$ within I day after the IV fluid was unintentionally withdrawn. We re-administered our strategy plus dexamethasone. The next day his BUN/Cr dramatically declined to $17 / 0.35 \mathrm{mg} \%$. Gradually his renal functions resolved within 4 days.

Conclusion: In some circumstances, haemolysis can be overcome by conservative treatment to avoid redoing percutaneous or surgical closure. 


\title{
Abstract no: 1252 \\ Radiofrequency perforation in the treatment of pulmonary atresia in an intact ventricular septum: Challenges in the cath labs of developing countries
}

\section{Sonia El Saiedi, Amal El Sisi, Wael Lotfy, Wael Attia and Osama Abd El Aziz}

Cairo University Children Hospital, Cairo, Egypt

Background: Pulmonary atresia with intact ventricular septum is an infrequent disorder with significant morphological heterogeneity. The use of percutaneous RF-assisted perforation of the atretic valve and subsequent balloon dilation provides an easy, but expensive overall procedure, that is expected to establish antegrade flow successfully through the pulmonary valve in most patients.

Methods and material: Twenty patients presenting with PA-IVS at Cairo University Children Hospital (CUCH) were taken for RF perforation using Baylis RFP100 generator with trials for cost limitations. Cost limitation methods to minimize expenses: We stopped using the Protrack microcatheter and replaced it in most cases by the "wire tracks a wire" technique. We also replaced the use of the micro snare from the arterial end to mark the pulmonary valve by looping a coronary wire. Telescopic Luma catheter was replaced by using Mullin long sheath $5 \mathrm{~F}$ or $6 \mathrm{~F}$ through which the $4 \mathrm{~F}$ catheter is passed. We also fixed the wire, and tried to limit the number of balloons by replacing the Tyshak mini balloons (which were not always available) with readily available regular coronary balloons. In case of unavailable 4F sheath with special curve we mostly used either 4F VER and reshaped it.

Results: Successful opening of the atretic valve with cost limitation was successful in $70 \%$ of cases. We resorted to a hybrid procedure with the surgeon opening the chest and fixing the sheath in RV directed towards the pulmonary valve in case of failed peripheral vascular access.

Conclusion: Curtailing cost limitation is essential in developing countries and innovative ideas to reduce cost are essential especially if it provides the same rate of success.

\section{Abstract no: 1255}

Normal growth of fetal cardiothoracic structures during the late Ist/early 2nd trimesters

\author{
Angela McBrien", Lisa Howley", Yuka Yamamoto", Akiko Hirose", Priya Sekar", Venu Jain", Tarek Motan",t, Jean Trines", Winnie Savard" \\ and Lisa Hornberger" \\ "Fetal and Neonatal Cardiology Programme, Department of Paediatrics, Division of Cardiology, University of Alberta, Edmonton, Alberta, Canada \\ \#Department of Obstetrics and Gynecology, University of Alberta, Edmonton, Alberta, Canada \\ tWomen's \& Children's Health Research Institute, Mazankowski Alberta Heart Institute, Alberta, Canada
}

Background/hypothesis: Fetal echocardiography is used increasingly early in pregnancy to screen for congenital heart disease (CHD). Data are lacking for normal growth of the fetal heart in the late Ist/early 2nd trimester. Fetal weight increases in a non-linear fashion and we hypothesise that growth of cardiothoracic structures is also non-linear.

Materials and methods: 197 healthy pregnant women were prospectively recruited to have detailed echocardiography between 8 and I4+6 weeks gestation. Atrial, ventricular, heart, chest, pulmonary artery and ascending aorta dimensions were measured. Statistical analysis included ANOVA.

Results: There was non-linear growth of all measured structures, with faster growth after 12 weeks gestation. From 8 to 10+6 weeks gestation, 95\% (20/2I) had a pericardial effusion. Heart and atrial size relative to chest size decreases significantly over time (Table I). There is no difference between right versus left ventricle and main pulmonary artery versus ascending aorta dimensions at any stage $(p>0.05)$

\section{TABLE I: Ratios of heart and atrial dimensions to chest size}

\begin{tabular}{|c|c|c|c|}
\hline Parameter & $\begin{array}{c}\text { Group I } \\
\text { (8-10+6 weeks) }\end{array}$ & $\begin{array}{c}\text { Group } 2 \\
\text { (I I-I } 2+6 \text { weeks) }\end{array}$ & $\begin{array}{c}\text { Group } 3 \\
\text { (13-14+6 weeks) }\end{array}$ \\
\hline & $\begin{array}{c}\text { Mean } \pm \text { SD } \\
n=21\end{array}$ & $\begin{array}{c}\text { Mean } \pm \text { SD } \\
n=81\end{array}$ & $\begin{array}{c}\text { Mean } \pm \text { SD } \\
n=95\end{array}$ \\
\hline Heart: Chest diameter & $0.40^{*} \pm 0.03$ & $0.34 \pm 0.03$ & $0.34 \pm 0.03$ \\
\hline Left atrium: Chest circumference & $0.19^{*} \pm 0.02$ & $0.16 \pm 0.02$ & $0.16 \pm 0.02$ \\
\hline Right atrium: Chest circumference & $0.20^{*} \pm 0.02$ & $0.16 \pm 0.02$ & $0.16 \pm 0.02$ \\
\hline
\end{tabular}

"Group I vs. 2 and 3, $p<0.05$.

Conclusions: We have documented growth of cardiothoracic structures during the late first/early second trimesters and established normal values. Pericardial effusion is almost universal in early pregnancy. The relatively large heart in the late-first trimester may relate to atrial size, possibly reflecting the importance of atrial function to filling the non-compliant early fetal ventricles. These data provide insight into cardiac development and should assist in early diagnosis of CHD. 
Abstract no: 1278

Ante-natal fetal cardiac screening: Use of outflow tract views improve detection of transposition of great arteries (TGA) and truncus arteriosus (TA): Experience of 2 regional fetal cardiac units and a single paediatric tertiary cardiac centre

\author{
Joyce Su-Ling Lim*,\#, Sharon Clark,\#, Devender Roberts" and Gordon Gladman*,† \\ "Alder Hey Children's Hospital, Liverpool, United Kingdom \\ \#Liverpool Women's Hospital, Liverpool, United Kingdom \\ 'St Mary's Hospital, Manchester, United Kingdom
}

Introduction: Outflow tract assessment of fetal hearts was introduced in the U.K. in 2008. We compared detection of TGA and Truncus Arteriosus (TA) in our region before and after the introduction of the new recommendation.

Methods: Retrospective review of fetal medicine and cardiac database at 2 regional fetal cardiac units and a single paediatric tertiary cardiac centre between January 2001 and June 2012. The detection rates for TGA and Truncus Arteriosus in babies scanned between 2001 - 2008 (before the introduction of the mandatory national outflow tract guidelines) and after 2008 were compared. A survey of practice at the referring obstetric units was also performed. Results:

TABLE I: Antenatal detection percentage

\begin{tabular}{|c|c|c|}
\hline & $\begin{array}{l}\text { Before mandatory outflow tract guidelines } \\
\text { (January 200I - December 2008) }\end{array}$ & $\begin{array}{l}\text { After mandatory outflow tract guidelines } \\
\text { (January } 2009 \text { - June 20 I 2) }\end{array}$ \\
\hline TGA & $15 / 120(12.5 \%)$ & 25/76 (32.9\%) \\
\hline Truncus arteriosus & $5 / 28(17.9 \%)$ & $13 / 26(50 \%)$ \\
\hline $\begin{array}{l}\text { Number of obstetric units using } 4 \text { chamber } \\
\text { as well as outflow tract views }\end{array}$ & I5/27 units (55\%) & All units \\
\hline
\end{tabular}

Prior to the mandatory national guidelines, only I5/27 obstetric units within the region perform outflow tract views for fetal cardiac screening. Now all obstetric units routinely perform 4-chamber as well as outflow tract views.

Conclusions: There is significant improvement of ante-natal cardiac detection of outflow tract abnormalities with introduction of the mandatory outflow tract views. It is hoped that as the screening sonographers get more used to the outflow tract views, and with more dedicated training of sonographers, the detection rate will increase further.

\title{
Abstract no: 1289 \\ Asymmetric right cardiac chambers enlargement in fetuses: A response to fetal hypoxia after the 30th week?
}

\section{Pedro Osvaldo Weisburd, Esteban Roberto Vazquez, Juan Pablo Feldman, Rodrigo Egues, Rolando Gomez and Graciela Citate}

Hospital de Niños Sor María Ludovic, La Plata, Argentina

Introduction: Fetal hypoxia (FH), with or without intra-uterine growth restriction (IUGR) evaluated by Doppler ultrasound (DU), was defined by measuring the ratios between the index of the middle cerebral (MCA) and umbilical arteries (UA) $(\mathrm{Rc} / \mathrm{u})<1$. The lack of symmetry between the cardiac chambers in favour of the right chambers (relation RA/LA and RV/LV >I.5 - I) not detected before the 30th week of gestational age (GA) without cardiac malformation (CM) could indicate the presence of a functional anomaly.

Objectives: The asymmetric right predominant cardiac chambers (ARPCC) manifested after week 30 th could be an indicator of FH. This not occurs before 30 th weeks. We discuss the possible physiopathology.

Materials and methods: I 5 fetuses ( $\mathrm{f}$ ) were referred for ARPCC between week 30 - 37 (mean 32, 5) and I0 between week 26 - 29 (mean 27.1 ) with an Rc/u $<$ I. A complete echocardiogram and DU in the UA, MCA, aortic isthmus and A-wave of the venous duct was performed.

Results: Of the 15 fetuses, 4 (26, 6\%) had a weight above percentile 50 (P50); 4 had P30; and 7 (46,6\%) less than P5. All of them presented ARPCC greater than I.5 to I and Rc/u <I. The flow at the aortic isthmus was reversed in all of them. The A-wave was reversed in 8 (I00\%) without IUGR and 4/7 (57. I\%) less than P5. 6/7 (85, 7\%) with IUGR presented as a T2I. In I fetus coronary flow was increased. Of the I0 fetuses $<30$ weeks with a Rc/u index $<1$, none presented ACCD.

Conclusion: After 30th week, ARPCC without CM could indicate severe FH for which fetal DU should be performed. ARPC associated with RCIU < P5 can indicate de risk of T2I associated. The ARPC apparently does not present In fetuses less 30th week with $\mathrm{Rc} / \mathrm{u}<1$. 


\title{
Abstract no: 1307 \\ The absence of physiological shunts during the fetal period can help predict severe post-natal hypoxia in fetuses with transposition of the great arteries with intact ventricular septum
}

\author{
Laurence Vaujois, Isabelle Boucoiran, Christine Houde, Jean Claude Fouron and Marie Josée Raboisson \\ Saint Justine University Hospital Centre, Montreal, Quebec, Canada
}

Background: Transposition of the great arteries with intact ventricular septum (TGA-IVS) is amenable to complete repair with low mortality rate. However, some neonates may experience profound cyanosis leading to rapid haemodynamic compromise.

Objective: We evaluated whether the assessment of physiological shunts during the fetal period could help predict post-natal profound hypoxia in neonates with TGA-IVS.

Methods: Echocardiographic data of 35 fetuses with TGA-IVS were retrospectively reviewed. The size of the foramen ovale (FO), septum primum (SP), main pulmonary artery (MPA) and aorta were measured. Doppler characteristics and output in the MPA, aorta and ductus arteriosus (DA) were assessed. The net pulmonary output was calculated as (output in the MPA - output in the DA). Patients were divided into 2 groups based on post-natal saturation: Group I had peripheral saturation $<50 \%$ and Group 2 had saturation $\geq 50 \%$.

Results: Eleven of the 35 fetuses (31.4\%) were in Group I. Fetuses in group I, with respect to group 2, had smaller FO (2.93mm vs. 4.07mm, p=0.02). In addition, holo diastolic intermittent or persistent retrograde flow in the DA was observed in I0/II patients (9I\%) in Group I in contrast to only 6/24 patients $(25 \%)$ in Group $2(\mathrm{p}<0.0 \mathrm{I})$. Patients with decreased mobility of the septum primum or a thick septum experienced higher output in the DA $(0.7 \mathrm{I}$ v. $0.32 \mathrm{ml}$ min, $p=0.01$ ). There was a positive linear correlation between the size of the fossa ovalis and the net pulmonary output $(r=0.54, p=0.004)$.

Conclusion: A decrease in the size of the FO with retrograde holo-diastolic flow in the DA is predictive of severe post-natal hypoxia in fetuses with TGA-IVS. Patients with smaller FO experience a lower net pulmonary flow with higher output through the DA possibly related to greater pulmonary hypertension and should be flagged for a Rashkind immediately after birth.

\section{Abstract no: 1310}

Uhl's anomaly: A difficult prenatal diagnosis

Laurence Vaujois, Nicolas van Doesburg, Myriam Brassard and Marie Josée Raboisson

Saint Justine University Hospital Centre, Montreal, Quebec, Canada

Introduction: Uhl's anomaly is a rare form of congenital heart disease with partial or complete absence of right ventricular myocardium, parchment-like appearance of the right ventricular wall, often associated with tricuspid anomalies. Only 3 prenatal cases have been reported so far. We describe a fetus with Uhl's anomaly that initially mimicked Ebstein's disease.

Case report: A 22-year-old pregnant woman was referred for suspicion of congenital heart disease at 2 I weeks of gestation (WG). Initial echocardiographic evaluation concluded to apical displacement of the tricuspid septal leaflet compatible with an Ebstein's anomaly, with mild right ventricular (RV) and right atrial (RA) dilatation. The RV free wall was surprisingly thin and hypo kinetic. 2 additional muscular VSDs were also reported. There was no tricuspid regurgitation and pulmonary flow, and flow in the ductus arteriosus and ductus venosus (DV) were normal. At 27WG, significant cardiomegaly and profound A-waves in the DV were reported, with moderate tricuspid regurgitation and RA dilatation. The ductus arteriosus showed bidirectional flow reflecting decreased RV output. At 30WG fetal supra ventricular tachycardia at 230bpm occurred leading to fetal hydrops. There was no more antegrade pulmonary blood flow. The RV free wall was even more abnormally thin and Uhl's anomaly was suspected. The fetus demised at 3IWG.

Discussion: Uhl's anomaly is a rare cause of fetal RV dilatation and can be associated with tricuspid dysplasia or pulmonary atresia. A cautious analysis of the RV free wall showing an abnormally thin myocardium and decreased myocardial thickening should help making the differential diagnosis between tricuspid disease and Uhl's anomaly. The evolution is poor with low RV output, progressive dilatation of the right heart chambers and rhythm disturbances leading to fetal demise.

\section{Abstract no: 1333 \\ Intermediate and long term outcomes of device closure of patent arterial duct with severe pulmonary hypertension

\author{
Masood Sadiq*, Asif-Ur Rehman*, Najam Hyder*, Ahmad Usaid Qureshi*, Tehmina Kazmi* and Shakeel Ahmed Qureshi* \\ "The Children's Hospital and Punjab Institute of Cardiology, Lahore, Pakistan \\ \#Evelina Children's Hospital, London, United Kingdom
}

Background: A subset of patients with large patent ductus arteriosus (PDA) and severe pulmonary arterial hypertension (PAH) progresses to adulthood without established pulmonary vascular disease. Whether to close or not to close and how to close these PDAs is often a difficult decision.

Objectives: To evaluate the results of device closures of large PDA with severe PAH after 2 years of age and report their intermediate and long term outcome. Methods: Fifty seven patients with large PDA and severe PAH (PAP $>2 / 3$ rds systemic) with no desaturation were considered for closure. The reversibility of $\mathrm{PAH}$ was assessed with $100 \% \mathrm{O}_{2}$ and trial balloon/device occlusion. Mean age was $12.5 \pm 1.4$ (2 - 55) years and mean weight 27.4 \pm 2 . I (9 - 70) kg. The follow-up was primarily based on echocardiography while 4 patients with persistent $\mathrm{PAH}$ underwent repeat catheterisation.

Results: Fifty patients demonstrated a decrease in PAP and device closure was successful in $47 / 49$ (96\%). Three patients underwent surgery, 2 after unsuccessful device closure. Seven (12.3\%) patients did not demonstrate a decrease after trial occlusion. ADO was used in 30/49 (6I.2\%), MVSD device in I4 (28.6\%) and ASO in 5 ( I $0.2 \%)$ patients. There was a significant decrease in peak systolic PAP $(8 \mathrm{I} \pm 4 \mathrm{vs} .6 \mathrm{I} \pm 3 \mathrm{mmHg}, \mathrm{p}<0.00 \mathrm{I})$ and mean PAP $(63 \pm 2 \mathrm{vs}$. $46 \pm 2 \mathrm{mmHg}$, $p<0.00$ I) following device occlusion. The device embolised to left pulmonary artery (LPA) in I patient needing surgery. At a mean follow-up of $49.2 \pm 3.7$ (6 - 121 ) months, there were no deaths, late embolisation and aortic protrusion. Mild LPA stenosis in 2 patients did not progress. Significant PAH has persisted in $4 / 50$ (8\%) patients.

Conclusions: Device closure of large PDA severe PAH with Amplatzer devices is safe and effective. Despite symptomatic improvement, PAH may not regress in $8 \%$ of the patients. In addition to more diligent non-invasive assessment, an accurate measurement of pulmonary vascular resistance together with direct measurement of PAP after trial occlusion may help further exclusion of borderline patients. 


\author{
Abstract no: 1352 \\ A single centre experience \\ Jonathan Mervis", Michael Mullin", Michael Rigby", Anselm Uebing" and Alan Magee* \\ "The Royal Brompton and Harefield NHS Foundation Trust, London, United Kingdom \\ \#The Heart Hospital University College, London Hospital Trust, London, United Kingdom
}

Medium term follow-up of endovascular stenting for native and recurrent coarctation of the aorta:

Aim: To describe the complete single centre experience of transcatheter stenting of native and recurrent coarctation of the aorta (CoA).

Patients and methods: From December 2000 - October 201 I, 160 patients (92 male) underwent transcatheter stenting for coarctation of the aorta. 107 had native coarctation (67\%) and 53 recoarctation, II of whom had previously underwent balloon aortoplasty. Median age and weight at procedure were 20.5 years (range 6 - 65 years) and $63 \mathrm{~kg}$ (range 24 - II $6 \mathrm{~kg}$ ) respectively. Bare metal stents were used in I3I patients and covered stents in 29 (I $4 \%$ ). 4 patients had more than I stent inserted during the procedure. Follow-up data was available for 129 at a median interval of 56 months (range 4 months - 10 years).

Results: Invasive peak systolic gradient decreased from mean $28 \mathrm{mmHg}( \pm 13)$ to $5 \mathrm{mmHg}( \pm 6)$. Coarctation site increased from mean $6 \mathrm{~mm}( \pm 3 \mathrm{~mm})$ - $13 \mathrm{~mm}$ $( \pm 2.5 \mathrm{~mm})$. There were no deaths, $8(5 \%)$ patients suffered procedure-related complications (acute pulmonary oedema (I); para-aortic aneurysm (I); aortic rupture (I); embolised stents (5)). Right arm systolic blood pressure decreased from mean of I 50 $\pm 20 \mathrm{mmHg}-128 \pm \mathrm{I} 6 \mathrm{mmHg}[\mathrm{P}<0.000 \mathrm{I}]$. 75 (47\%) remain on anti-hypertensive medication. Echocardiographic Doppler derived gradient decreased from mean of $58.7 \mathrm{mmHg}( \pm 15.8 \mathrm{mmHg})-25 . \mathrm{mmHg}( \pm 14.3 \mathrm{mmHg})$ $[\mathrm{P}<0.000 \mathrm{I}]$. Eighty patients $(50 \%)$ had post procedural CT angiograms at approximately 3 months. Only I aneurysm was identified which was unchanged from the post procedure angiogram. There were no late complications.

Conclusions: Transcatheter stenting of native and recurrent coarctation of the aorta is a safe and effective therapeutic option in this age group with benefits maintained in the medium term. Complications tend to occur at the time of the procedure. Routine post procedural CT angiography does not appear to be necessary and the place of covered stents has still to be established.

\title{
Abstract no: 1356
}

Successful transcatheter closure of a patent ductus venosus with an Amplatzer vascular plug 2 in an infant

Jayneel Joshi, Belinda Jane Mitchell, S. Ahmad, C. Kock and F.F. Takawira

Steve Biko Academic Hospital, University of Pretoria, South Africa

Background: The ductus venosus is a embryological vascular structure that connects the umbilical vein with the inferior vena cava. The ductus venosus rarely persists $>2$ weeks after birth. If however it remains patent beyond this period and the resulting porto-systemic shunt is significant it can be an extremely rare cause of liver failure.

Method and material: We present a rare case of a large patent ductus venosus causing cholestatic jaundice and severe liver dysfunction in a 6-day-old neonate. Abdominal sonography demonstrated a large vascular channel adjacent to the ligamentum teres which was identified to be patent ductus venosus.

Results: CT angiography was unsuccessful due to technical difficulties and because of the deteriorating liver function a combined diagnostic and interventional catheterisation was planned. At catheterisation a 6F sheath was inserted into the right internal jugular vein. Angiography demonstrated a large patent ductus venosus measuring $6 \mathrm{~mm}$ at its widest diameter and $26 \mathrm{~mm}$ in length. Balloon occlusion was performed with a wedge pressure catheter in order to measure the portal pressures once the ductus was occluded, which showed no significant increase.

The decision was then made to occlude the ductus venosus using a $12 \mathrm{~mm}$ Amplatzer Vascular Plug Type II. The device was deployed with some initial occlusion to the normal hepatic venous drainage. It has therefore then retrieved into the sheath and redeployed with a good result.

Results: The child did well post catheterization with the liver functions recovering to within normal limits shortly after closure.

Abstract no: 1360

Long term outcome of balloon dilatation of valvular aortic stenosis as primary treatment: The need for reintervention

Jaana Pihkala and Talvikki Boldt

Children's Hospital, University of Helsinki, Helsinki, Finland

Background: Although balloon dilation of congenital aortic stenosis has become a primary therapeutic strategy, few data are available regarding long term outcomes.

Objectives: We evaluated the long-term (median 6.0, range 1.6 - 1 1.6 years) results of balloon dilation of severe or critical aortic valve stenosis in children in our institution where all our country's invasive paediatric cardiology is centralized.

Methods: Retrospective long term follow-up study comprised 47 children who underwent balloon aortic valvuloplasty (BAV) between January 2000 and December 2010. There were 20 neonates, 16 children aged I - 12 months, and eleven aged >12 months. We assessed clinical, catheterisation and echocardiographic outcome and need for reintervention.

Results: The median (range) age at dilatation was 0.1 I ( 0 - 16.8) years and weight $4.4(2.2$ - 84) kg. I 4 children received prostaglandins, 66\% had bicuspid aortic valve and $47 \%$ had other cardiac abnormalities. Median peak gradient before dilatation measured 60 (35 - II3) $\mathrm{mmHg}$ and after dilatation 17 (3 - 48 ) $\mathrm{mmHg}$ $(\mathrm{p}<0.000 \mathrm{I})$. Valve regurgitation before dilatation was graded as none or mild in all children; after dilatation as moderate in 7 ( I $5 \%)$; and as none or mild in others Mean balloon-to-annulus ratio measured 0.91. Complications in I5\% of patients included: transient pulse loss (4); transient haematuria (I); transient hypotension ( I); and pericardial tamponade and resuscitation (I) leading to neurological damage and death. One child died 2.5 months after BAV, after operation for CoA. Acute and long term mortality rates were $2 \%$ and $4 \%$, respectively. During median follow-up of 6 years, survival was $96 \%$ and freedom from reintervention $57 \%$. Twenty children had undergone reintervention: repeat balloon valvuloplasty in six (13\%), and surgery in 14 (30\%) including valvotomy ( $n=5$ ), Ross operation $(n=6)$, and aortic valve replacement $(n=3)$.

Conclusions: In our centre, balloon dilatation of aortic stenosis has been effective with low complication and mortality rates. Reintervention is frequently needed. 


\title{
Abstract no: 1368
}

\section{Acute dissection and pseudoaneurysm with transcatheter patent arterial duct device occlusion}

\section{Supratim Sen and Philip Roberts}

Heart Centre for Children, The Children's Hospital at Westmead, New South Wales, Australia

Background: Transcatheter device occlusion of PDA is a well-established and safe procedure with a high success rate. Previous reports describing pseudoaneurysms as complications with PDA devices have alluded to femoral artery pseudoaneurysms at the vascular access site. A literature review did not identify reports of acute dissection and pseudoaneurysm formation during transcatheter PDA occlusion.

Method and material: A 3.I kg 74-day-old infant with a moderate ASD, PDA and pulmonary valve stenosis was planned for transcatheter balloon pulmonary valvotomy (BPV) and PDA device closure. PV annulus measured $8 \mathrm{~mm}$. An aortogram with a 4F vessel sizing pigtail with the end cut off showed a long and tubular PDA with a slight constriction at the pulmonary end.

Results: BPV was performed with a $9 \mathrm{~mm} \times 3 \mathrm{~cm}$ Tyshak II balloon. A 4-4 ADO II AS device was deployed from the aortic side. The device was however freely mobile on stability testing and pushed through the duct into the MPA with easy retrieval of the fully deployed device back into the aorta. The delivery system and unreleased device were removed. Repeat angiography into the PDA with a cut pigtail catheter demonstrated dissection and pseudoaneurysm of the duct with 2 exit points into the MPA. Transthoracic echocardiography confirmed a tissue flap at the proximal pulmonary end of the duct and a pseudoaneurysm which prolapsed into the MPA. The infant was referred for surgical PDA ligation. Post ligation, there was no residual PDA with resolution of the pseudoaneurysm.

Conclusions: The dissection could have occurred during BPV, during retrieval of the occlusion device or with positioning the cut vessel sizing pigtail during the 2nd aortogram. We feel the injury most likely occurred with the cut pigtail catheter. This highlights the risks associated with the sharp edges of a cut catheter.

\author{
Abstract no: 1372 \\ Nam Kyun Kim*, Su-Jin Park*, Jo Won Jung*, Lucy Youngmin Eun*, and Jae Young Choi* \\ "Congenital Heart Disease Centre, Severance Cardiovascular Hospital, Department of Paediatrics, \\ Yonsei University College of Medicine, China \\ \#Department of Paediatrics, Gangnam Severance Hospital, Yonsei University College of Medicine, China
}

Feasibility and safety of transcatheter closure of atrial septal defect in small children weighing I0kg or less

Background: Transcatheter closure of ASD has been accepted as a standard treatment for patient with haemodynamic significance in children and adults. Little is known about very small children and infants with poor weight gain and symptoms with congestive heart failure.

Materials and methods: From April 2004 - March 2008, 316 patients underwent transcatheter closure of ASD using Amplatzer septal occludder ${ }^{\circledR}$ (ASO, Golden Valley, MN) in our institute. Among them 94 patients were weighing $10 \mathrm{~kg}$ or below. The indication of early treatment in each group was symptoms of congestive heart failure with volume overload of right side heart. We analysed the demographic data, clinical characteristic and outcome of the patients.

Results: There were 28 males and 66 females. Median age was 15 months (7 - 15 months) and average weight was $9.1 \mathrm{~kg}$ (5.0kg - I0 kg). Median ASD size was $15 \mathrm{~mm}(10 \mathrm{~mm}-24 \mathrm{~mm}) .4$ patients were sent to surgery because of the encroaching mitral valve by LA disk after device placement. The procedure was successful in rest of the patients. There was no mortality in all patients. Complete closure rates at discharge were $81.8 \%$. Only I minor complication was noted during the procedure (transient arrhythmia). The mean hospital stay was 4.7 days.

Conclusions: Transcatheter closure of secundum ASD with the ASO is technically feasible, safe and effective even in very small children and infants < IOkg. Meticulous patient selection is of critical importance to avoid undue invasive procedures in this unique group of patients.

\section{Abstract no: 1376 \\ Ductal closure using Amplatzer duct occluder type II additional sizes: Early experience}

\section{Lungile Pepeta and Adele Dippenaar}

Port Elizabeth Hospital Complex, Walter Sisulu University, Port Elizabeth, South Africa

Background: Various devices have been developed for percutaneous closure of PDAs in all patients but the small infant. Such devices lead to either pulmonary artery or descending aorta obstruction.

Methods: Records of patients undergoing ductal closure were reviewed. Demographics; haemodynamic and angiographic characteristics including size, shape, length and aortic diameter; device to close the duct and closure approach, screening time; complications; and outcomes were recorded.

Results: From June 20 I I - July 20 2; 22 patients (I I females and II males) were assigned to percutaneous closure using Amplatzer Duct Occluder II Additional Sizes (ADO II AS). Patients' mean age was 9 months (range, I month - 60 months), and mean weight was $5.4 \mathrm{~kg}$ (range $1.1 \mathrm{~kg}-12.9 \mathrm{~kg}$ ). The QP:Qs ratio mean was 2.2 (range, I.I - 4.89) and pulmonary vascular resistance mean was $1.88 \mathrm{WU}$ (range, $0.12-7.12 \mathrm{WU}$ ). The ductal size mean was $1.9 \mathrm{~mm}$ (range, $0.6 \mathrm{~mm}$ 3.2mm): Krichenko Type A duct (I); type B (2); type C (2); type D (I); and type E (5). The screening duration mean was 21.1 minutes (range, 9 - 45.3 minutes). 9 patients were occluded with ADO II AS $04 \times 06 \mathrm{~mm}$ device; 8 with $05 \times 06 \mathrm{~mm} ; 2$ with $03 \times 06 \mathrm{~mm}$; I with $03 \times 04 \mathrm{~mm}$; I with $04 \times 02 \mathrm{~mm}$ and I with $05 \times$ $02 \mathrm{~mm}$. In 19 patients the device was deployed via the pulmonary side and in 3 patients, via the aortic side. In I patient, the device dislodged to the pulmonary arteries immediately following deployment, with successful retrieval. Complete ductal occlusion was achieved in all (I00\%) other patients ( $n=2 \mid$ ) before discharge (day I).

Conclusion: The ADO II AS is a safe and effective device for closure of small ducts in even smaller infants. There is minimal risk for aortic and pulmonary artery obstruction with the device. 


\author{
Abstract no: 1386 \\ Transcutaneous aortic valve replacement: Initial experience in a developing country \\ Stephen Brown, Hennie Theron, Edwin Turton, Coert de Vries, Francis Smit, Johan Jordaan, Ruan Botha and Jacques van Rensburg \\ University of the Free Sate, Bloemfontein, South Africa
}

Aim: Critical aortic stenosis is a common structural heart disease in the elderly and symptomatic stenosis has a mortality of $50 \%$ over 24 months. Surgery for this group carries 3 - 15\% mortality. Transcutaneous aortic valve insertion (TAVI) is a non-invasive alternative to surgery. The aim of this study was to assess the initial experience of TAVI in a high risk aortic stenosis population of central South Africa.

Methods: Prospective study of all patients undergoing a TAVI procedure over a 12 month period. Seventeen patients with a median age of 79.6 year (range: 62.8 - 87.9) were included. Median aortic valve area and gradient were $0.6 \mathrm{~cm}^{2}$ (range: $\left.0.5-1.5\right)$ and $70 \mathrm{mmHg}(46-120$ ), respectively. Co-morbidities were present in all with a median Euroscore of 15 and predicted STS mortality of $21.4 \%$.

Results: Vascular access was percutaneously gained in 16 and surgically in I patient. All valves were successfully implanted: $26 \mathrm{~mm}(\mathrm{n}=6$ ), $29 \mathrm{~mm}$ ( $\mathrm{n}=7$ ) and $31 \mathrm{~mm}(\mathrm{n}=4)$. Procedure time ranged from 45 - 160 minutes. No procedural or peri-procedural deaths occurred. Aortic valve peak gradient decreased significantly to a median of $0 \mathrm{mmHg}$ (range, 0 - 3l) ( $\mathrm{p}<0.000 \mathrm{I}$ ). Mean hospital stay was $3.9 \pm 1.3$ days. 4 patients (23\%) required permanent pacemaker insertion. NYHA class symptomatology improved significantly $(p<0.001)$ during follow-up. There were 2 late deaths, not related to the procedure $(78$ and 93 days, respectively)

Conclusion: Our results show that TAVI is feasible in our population. The procedure is successful and follow-up shows that stable device position is maintained in all patients. Clinical and haemodynamic improvement could be demonstrated in all patients. Short term mortality and morbidity show that TAVI is an acceptable alternative to conventional surgical valve replacement in this high risk group of patients.

\title{
Abstract no: 1407
}

Radiofrequency ablation of multifocal right atrial tachycardia facilitated by 3-D mapping and robotic assistance in a patient with repaired Tetralogy of Fallot

Faizel Lorgat, Evan Pudney and Helena van Deventer

Chris Barnard Memorial Hospital, Cape Town, South Africa

Background: Patients with Congenital Heart Disease often have complex cardiac arrhythmias with extensive underlying substrate. Significant structural variations in the underlying anatomy provide further challenges to the success of ablation therapy. Cumulative radiation exposure of the operator is an added concern. Materials and methods: A 50-year-old male patient with repaired Tetralogy of Fallot presented with incessant tachycardia and heart failure. The cardiac ejection fraction was reduced at 43\%. A cardiac electrophysiological study was performed and a Multifocal Right Atrial Tachycardia was identified. A detailed 3-D map of an enlarged Right Atrium was generated using an integrated robotic sheath and RF Ablation catheter (LYNX, Hansen Medical) and Ensite Velocity (St Jude Medical). Arrhythmogenic foci were identified along the Crista Terminalis and around the sinus node. The sinus node was carefully tagged on the 3-D map. RF ablation was remotely performed with precision guidance of the Lynx RF ablation catheter.

Results: The arrhythmogenic foci were successfully ablated and tachycardia was rendered non-inducible. Sinus node function was unchanged post ablation. After 2 months of follow-up the patient remains in stable sinus rhythm and LV function has recovered completely with the EF now $60 \%$.

Conclusion: Ablation of complex and challenging cardiac arrhythmias is greatly facilitated by 3-D mapping and robotic assistance. These technologies also improve safety and reduce operator fatigue and radiation exposure.

\section{Abstract no: 1408}

Radiofrequency ablation of symptomatic premature ventricular complexes (PVCs) in the young patient

\section{Faizel Lorgat, Evan Pudney and Helena van Deventer}

Chris Barnard Memorial Hospital, Cape Town, South Africa

Background: Although often benign, PVCs can cause severe symptoms in some patients and may induce ventricular dysfunction. Drugs are frequently not efficacious in this condition and have major side effects and toxicity which is particularly relevant in the young patient.

Materials and methods: Two young patients presented with symptomatic high grade PVCs. The first, a 21 -year-old male patient was almost permanently in a bigeminal rhythm with bizarre atypical LBBB morphology ectopics. The ensuing compensatory pauses produced sinus bradycardia. The 2 nd a I 0 -year-old female had I 233 PVCs per hour on Holter monitoring. These had positive inferior axis and morphology consistent with a RVOT source. Aside from mildly dilated Right Ventricular Outflow tracts on the echocardiogram, both had otherwise structurally and histologically normal hearts as determined by RV Angiography, Cardiac MRI and RV Biopsy. Both patients underwent Cardiac EP studies. In the first patient, a 3-D map was constructed and an activation map identified an arrhythmogenic focus on the free wall of the RV adjacent to the tricuspid annulus. This was ablated with robotic assistance. An arrhythmogenic focus was identified in the 2 nd patient in the RVOT and manually ablated.

Results: Both patients were rendered completely asymptomatic off all drug therapy. Follow -p ECG and Holter studies confirmed elimination of the PVCs. Conclusion: Radiofrequency ablation is an effective and safe treatment of symptomatic PVCs in the young patient. 


\author{
Abstract no: 1419 \\ Assisted conception and the risk of congenital heart disease: A case control study \\ Piers Daubeney",\#,, Rodney Franklin", Susie Schofield", Victoria Doughty", Nicole Van Stiphout", Mark Johnson ${ }^{\#, t}$ and Paul Cullinan",\# \\ "Royal Brompton Hospital, London, United Kingdom \\ \# Imperial College, London, United Kingdom \\ tChelsea and Westminster Hospital, London, United Kingdom
}

Background: Epidemiological studies suggest a higher prevalence of congenital malformations in children conceived through assisted reproductive technologies (ART). Few studies address congenital heart disease (CHD) specifically and most examined data from registries. We examined the relationship between CHD and assisted conception using a case control design set in a specialist paediatric cardiac service in the UK.

Methods: Between April 2010 and July 20II the parents of children attending paediatric cardiology clinics at the Royal Brompton Hospital, London were invited to complete a questionnaire which enquired into the nature of their child's conception, route for their referral and a number of potential confounding exposures. "Cases" were defined as children diagnosed with one or more carefully defined CHDs and "controls" as those discovered not to have CHD. Assisted conception technologies included any of the following procedures/treatments: ovulation induction, in vitro fertilisation, intra-cytoplasmic sperm injection or intrauterine insemination.

Results: Of the 2834 eligible patients 2255 (80\%) were recruited, 894 of which were newly referred patients with adequate data; half were cases (n=4I0, 46\%). The remaining had other heart abnormalities; cardiomyopathy (2.1\%), arrhythmia (3.4\%) and acquired heart disease (3.0\%). The overall prevalence of ART was $5.4 \%(n=44)$. Logistic regression analysis demonstrated a non-significant increase in the crude odds for the use of ART (I.2I, 95\% CI 0.66, 2.22). After adjustment for gestation, multiparity and maternal age, the estimate was reduced (OR 1.00, 95\% Cl 0.5 I, 1.95, p=0.993). An increased prevalence was found in a number of the subgroups, the highest amongst those with a functionally univentricular heart (16.7\%), although none were significant.

Conclusions: We found no increased risk of CHD in those conceived by assisted procedures. An increased risk was found in some subgroups, albeit nonsignificant, warranting further investigation.

\title{
Abstract no: 1428
}

Angioplasty procedure in aortic coarctation

\section{Maria Fernanda Biancolini, Julio Cesar Biancolini, Victorio Lucini, Adelia Marques, Ines Martinez, Judith Ackerman, Adriana Olive,} Jesus Damsky Barbosa and De Dios Ana

Pedro De Elizalde Children's Hospital, Buenos Aires, Argentina

Aim: Follow-up of patients ( $\mathrm{p}$ ) with Aortic Coarctation (AoCo) post angioplasty (BCA) procedure.

Methods: Retrospective analysis of 47 patients post angioplasty procedure, grouped by severity: Mild <20mmHg; moderate 20 - 40mmHg; and severe $>40 \mathrm{mmHg}$. Topics reviewed: Age at diagnosis; clinical manifestations (cardiac insufficiency (Cl); arterial hypertension (AH); defect extension (localised, extensive, isthmus hypoplasia); associations; initial treatment (catheterisation or surgery); and long term evolution. Multivariable statistical analysis $T$ test $\mathrm{p}<: 0.05$.

Results: 47 patients with AoCo post BCA. Age at diagnosis: <I month (I I), I - 2 years (I7), >2 years (I3). Age at BCA: Median $8 \pm 8$ month. Clinical exam: 19 patients $\mathrm{Cl}$ and 8 patients $\mathrm{AH}$; I patient $\mathrm{AH}$ without $\mathrm{Cl}$. EKG: Right ventricular hypertrophy I3), left 9| I). Associations: Ventricular septal defect: (I3 = perimembranous (5), muscular (7), subaortic (I)); ductus arteriosus (8); mitral stenosis ( 1 ); mitral insufficiency (3); aortic insufficiency (3); and right aberrant subclavian artery (I). Anatomic types: Localised (4I): membranous (6); fibromuscular (35); extensive (I); Hypoplasia of transverse aortic arch (7/47). Severity: Moderate ( I 8); severe (29). BCA: 47 patients required 52 procedures: I BCA (44); 2 BCA (4); native AoCo (2); 4 BCA (I); stent (I). Efficiency: Efficient in 38/47 (80.8\%); not efficient in 8; I patient died of cardiogenic shock during the procedure. Gradient post procedure: Dropped from $50 \pm$ I $8 \mathrm{mmHg}$ to I4 \pm I $\mathrm{mmH}$ ( $\mathrm{p}$ :

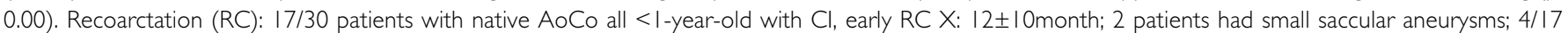
patients post-operatively RC (23\%), I patient required stent and 3 patients another BCA.

Conclusion: Angioplasty is an efficient recourse to solve complicated types of aortic coarctation and emergences in infants (localised, without isthmus hypoplasia). This procedure in infants has high risk of recoarctation (57\%) and saccular aneurysms (4.2\%). Therefore, except in those special situations, it's not recommended as Ist therapeutic choice.

\author{
Abstract no: 1437 \\ A meta-analysis on the efficacy and safety of transcatheter device closure of ventricular septal defects (VSD) \\ Swee Chye Quek, Bee Choo Tai and Lin Qi Yang \\ National University of Singapore, Singapore
}

Background: Advances in interventional techniques now allow for transcatheter treatment of some VSDs. Unlike ASDs widespread use of this option is limited due to several factors, one being concerns about adverse events. We performed a systematic analysis to look at outcomes and complications associated with use.

Method: A Pubmed search for series in English on device closure of VSD from 2003 - June 2012 was performed. We excluded small series which were included in multi-centre studies so as not to double count, and also patients who had acquired VSD following myocardial infarction. The random effect model was used to obtain pooled estimates of success and complications.

Results: A total of 37 publications comprising 4406 patients with VSDs (perimembranous=3758; muscular=4 I 9; intracristal=47; doubly committed subarterial=36; multiple=16; post-surgical 123; unclassified=7) were included in this analysis.

The age of patients ranged from 3 days to 84 years with the mean age ranging from $0.4-37.9$ years at the time of surgery. The pooled estimate of success of device implantation was $96.6 \%$ (95\% Cl:95.7-97.5; heterogeneity test $\mathrm{p}<0.00 \mathrm{I}$ ). Complications included residual shunt, rhythm abnormalities, valvular defects and others. 
Discussion: Our analysis suggests that transcatheter device closure of VSD is safe and yields good results. However, this study does not analyse different devices individually, and it does not segregate age range and different VSD types.

Conclusion: Transcatheter closure of VSD appears a feasible alternative to surgery (with very high success and low complication rates). However, there are several complications which require attention. Further data stratifying type of VSD, age range of patients and prevention of complications would be useful before this can be recommended for routine treatment of haemodynamically significant VSD.

\section{Abstract no: |44|}

Fetal aortic valvuloplasty technical aspects and factors influencing post-natal outcome

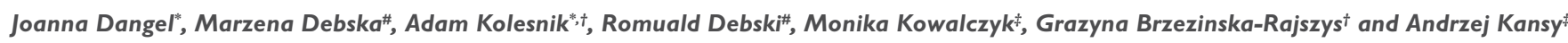

"Medical University of Warsaw, Centre for Post-graduate Medical Education, Children's Memorial Health Institute, Warsaw, Poland

\#The Centre of Postgraduate Medical Education, Warsaw, Poland

†Children's Memorial Health Institute, Warsaw, Poland

¥Children's Memorial Health Institute, Warsaw, Poland

Objectives: There are still many queries concerning fetal aortic balloon valvuloplasty. The aim of this study was to evaluate early experience and find the main factors influencing the post-natal outcome.

Material and methods: Between June $201 \mathrm{I}$ and July 2012 fetal aortic valvuloplasty was performed in I0 fetuses and diagnosis was as follows: Evolving HLHS (8); severe heart failure with fetal hydrops (2). The mean age of treatment was 24 weeks. Maverick 2 catheters were used, with balloon size slightly $>$ AoV. Six procedures were performed under general anaesthesia, 4 under mothers' intravenous and fetal analgesia with fentanyl.

Results: All aortic valves were successfully dilated, without major complications during and just after the procedure. Pericardial punction was necessary in 2 , adrenaline injection in 2. One emergency caesarean section was performed due to placental abruption at the 2 nd day after the procedure, I fetus died at the end of pregnancy due to obstetrics reason. Two fetuses are still in utero. Aortic diameter was within lower limits of normal in all those children. Three neonates with big LV of poor function, had balloon valvuloplasty just after birth. Conversion to the uni-ventricular circulation was attempted in 2 of them, and the $3 r d$ is still a neonate. All 3 of them had severe heart failure. Three Norwood operations were performed without complications.

Conclusions: Fetal aortic valvuloplasty is technically possible and can be performed by the experienced team without major complications. Good aortic development was observed in all children, what made the Norwood procedure easier in neonates with hypoplastic left ventricles. Neonates with borderline left ventricle with high gradient across the aortic valve, were the most difficult group of patients. After our early experience we think, that early aortic valve replacement, what means neonatal Ross-Konno operation, should be considered to improve long term outcome of children after pre-natal aortic valvuloplasty.

\section{Abstract no: 1467 provider knowledge \\ Sarah Heinonen*, \#, Neda Mulla", Jennifer Newcombe ${ }^{\#}$ and Sarah (Jodi) Shaefer ${ }^{\dagger}$ \\ "University of Kansas Medical Centre, Kansas City, United States of America \\ \#Loma Linda University Medical Centre, Children's Hospital, California, United States of America \\ Johns Hopkins University, Baltimore, United States of America}

FERP-fetal echocardiography referral project: The dissemination of guidelines aimed to improve

Background: Although clinical practice guidelines do exist regarding fetal echocardiography referrals, these guidelines are often not utilised in practice due in part to lack of provider knowledge.

Aim: The primary purpose of this 6-week Quality Improvement (QI) Research Project was to increase the knowledge of obstetrical providers on the guidelines for fetal echocardiography referrals.

Method and material: A 3-phased intervention project using a non-experimental descriptive design methodology was implemented, employing both pre and post project surveys to a convenience sample of obstetrical providers within a 5 I-mile radius of a specified Southern California zip code.

Results: A paired-samples t-test was used to evaluate the statistical significance of pre- and post-project obstetrical provider scores. Study findings indicated a positive correlation between the use of an education intervention, implemented by an advanced practice nurse and her team, utilising a multi-modal approach and increased provider knowledge on Fetal Echocardiography Guidelines.

Abstract no: 1482

Stenting the patent ductus arteriosus in neonates with critical congenital cyanotic heart disease in a developing country

Stephen Brown, Carri-Lee Greig, Daniel Buys and Francis Smit

University of the Free State, Bloemfontein, South Africa

Background: Critical cyanotic heart disease in newborns is life-threatening and difficult to manage. Systemic to pulmonary artery shunts are used as temporary palliative interventions. This is associated with increased morbidity and mortality. Patent Ductus Arteriosus (PDA) stenting has been introduced as a non-surgical alternative. The aim of this study was to determine the short and medium-term outcomes of PDA stenting in neonates with duct-dependent pulmonary circulations in central South Africa.

Materials and methods: Sixteen neonates with duct-dependent pulmonary circulation requiring PDA stenting were included in a retrospective review from September 2005 - March 2012. In 3 cases stenting was not performed due to inability to obtain vascular access. Stenting was performed via the femoral artery and/or vein. Standard balloon expandable coronary bare metal stents were used off-label. Follow-up included clinical assessment and echocardiography. 
Results: Thirteen cases were successfully stented at first attempt. 5 underwent concomitant Rashkind septostomy. Diagnoses consisted of Pulmonary Atresia $(n=9)$ and Tricuspid Atresia $(n=4)$. Median age at procedure was 2 days (range: 0 - 23) and median weight 3.0kg (range: I.5 - 4.3). Number of stents used: I $(n=3) ; 2(n=9) ; 3(n=1)$. Implanted stent diameters were: $3.5 \mathrm{~mm}(n=4) ; 4 \mathrm{~mm}(n=7) ; 4.5 \mathrm{~mm}(n=1) ; 5 \mathrm{~mm}(n=1)$. Median saturations were $80 \%($ range: $52-92)$ pre-procedure and $90 \%$ (range: 64-97) post-procedure $(p=0.07)$. Peri-procedural complications included stent migration into pulmonary artery ( $\mathrm{n}=\mathrm{I}$ ), femoral artery compromise $(n=1)$ and temporary dysrhythmia $(n=3)$. There was no periprocedural mortality. All patients received low dose acetylsalicylic acid following the procedure. At last follow-up (median 136 days; range: 7 - 334), balloon angioplasty $(n=I)$ and re-stenting ( $n=I)$ of stenotic stents had been performed. Three cases had proceeded to palliative surgery. 4 cases had demised at a median of 9.5 days (range: 7- 14 ) post-procedure, secondary to septicaemia ( $\mathrm{n}=\mathrm{I}$ ), airway obstruction $(n=1)$, pneumonia $(n=1)$ and severe $\operatorname{HOCM}(n=1)$. All deaths were unrelated to the procedure.

Conclusions: PDA stenting is a safe and effective alternative to surgical shunts as a palliative procedure for neonates with duct-dependent pulmonary circulation.

\section{Abstract no: | 489 \\ Giant intracardiac haemangioma diagnosed in utero \\ Claudeen Scott, Gurur Biliciler-Denktas, Kenneth Moise, Anthony Johnson, Michael Bebbington, Mousumi Moulik, Ashish Banker and Michael Hines}

UT Medical School at Houston, Houston, United States of America

Background: We describe a case of a fetus diagnosed at GA 3 I weeks with a large right atrial cavernous capillary haemangioma who had elective delivery by Caesarean section and successful elective resection of tumour at I week of life.

Material and method: A 36-year-old G3P200I mother was referred for a fetal echocardiogram when a mass was visualised on routine anatomy scan at 3 I weeks gestation. Fetal echocardiogram was significant for a large loculated visualised mass occupying the majority of the right atrial cavity with sparing of the posterior 3rd of the right atrium. There was no obstruction to the tricuspid valve inflow, systemic venous return or coronary sinus. There was a small pericardial effusion of no haemodynamic significance. The fetus was in sinus rhythm.

Results: Patient was followed weekly with echocardiograms. The night prior to delivery, the fetus was in atrial flutter in 2:I at a ventricular rate of 300/minute without hydrops fetalis. Rhythm has spontaneously converted to sinus before delivery. Delivery was uneventful with Apgar scores 8 and 9 . Echocardiogram postdelivery revealed a wide based large mass $(19.9 \times 21.3 \mathrm{~mm})$ in the lateral wall of the right atrium, attached to the right atrial appendage extending anteriorly to the aortic root. The mass had irregular borders with cystic formation inside in addition to echogenicity with blood flow with connection to the right coronary artery. Elective resection of the tumour was done at I week of age. Pathologic evaluation also confirmed the diagnosis. Patient was discharged home a few days after surgery.

Conclusions: Compared to the most frequently seen rhabdomyomas and less common teratomas and fibromas, cardiac haemangioma is one of the rarest types of benign cardiac tumours found in neonates. Fetal echocardiogram is an essential diagnostic tool for early detection, close monitoring and appropriate and early treatment.

\section{Abstract no: 1506 Masood Sadiq*, Asif-ur Rehman*, Ahmad Usaid Qureshi* and Shakeel Ahmed Qureshi" \\ "Punjab Institute of Cardiology and The Children's Hospital, Lahore, Pakistan \\ \#Evelina Children's Hospital, London, United Kingdom}

Covered stents in the management of native coarctation of the aorta: Intermediate and long term follow-up

Objectives: To evaluate the results of the use of covered Cheatham-Platinum (CP) stents in the management of native coarctation of the aorta (CoAo) and report intermediate and long term follow-up.

Background: Covered stents are being used increasingly in CoAo to reduce risk of aortic wall complications; however, there is limited data on the intermediate and long term outcome.

Patients and methods: Fifty nine covered CP stents were implanted in 56 patients. In 54 patients these were as primary treatment for severe CoAo with near atresia and complex CoAo and in 2 as rescue for complications related to bare stent implantation. The follow-up was based on echocardiography and computed tomography.

Results: Mean patient age was 22.25+ I.2 ( I I - 56) years and weight was 58.6+2.1 (32 - 99) kg. The systolic gradient across the CoAo decreased from mean of $5 \mathrm{I} .4 \pm 3.4-4.6 \pm 0.7 \mathrm{mmHg}(\mathrm{p}<0.000 \mathrm{I})$. The diameter of the CoAo segment increased from $4.69 \pm 0.20-15 . \mathrm{I} \pm 3.2 \mathrm{~mm}(\mathrm{p}<0.000 \mathrm{I})$. There was I death 3 days post-procedure due to cerebral anoxia. There was I dissection diagnosed 24-hours post procedure. At a mean follow-up of $45.9+3.9$ (3 - I20) months, all stents were patent and in good position on computed tomography. 4 (7.1\%) patients underwent successful re-dilatation. Antihypertensive medication was decreased or stopped in $37(66 \%)$ patients.

Conclusions: Covered CP stents may be used effectively as the therapy of choice in selected patients with severe CoAo. Aortic wall complications can occur even with covered stents. The stents can be redilated safely to keep pace with somatic growth. Covered stents provide a safe alternative to conventional stenting in the intermediate and long term.

\section{Abstract no: 1512}

Development of the left ventricle in pre-natally diagnosed left-sided obstruction

Hana Jicinska"\#, Pavel Vlasin" and Jiri Navratil"

"University Children's Hospital Brno, Czech Republic

${ }^{\#}$ Centre for fetal care, Brno, Czech Republic

Background: The asymmetry in 4-chamber view with small left ventricle (LV) in fetal heart could be caused by the obstruction in different levels of the left heart: by the presence of left superior vena cava draining coronary sinus (LSVC-CS), aortic stenosis (AS), coarctation of aorta (CoAo) or hypoplastic left heart syndrome (HLHS).

Aim: To follow-up the development of the LV in fetuses with left-sided obstruction. 
Material and method: The length of the LV was measured at the time of diagnosis pre-natally and after birth in echocardiographic evaluation in I 3 I fetuses with left-sided obstruction and small LV.

Results: Out of these I3I fetuses detected in II - 37 week of gestation: 42 of them were diagnosed with LSVC-CS; 30 with AS; 24 with COA; 2 I with HLHS; and 14 without final diagnosis (non-specified disproportion) due to early week of gestation. There were extracardiac abnormalities in 24 fetuses and chromosomal abnormalities in 20/131 fetuses. The pregnancy was terminated in 51 (39\%) fetuses: in HLHS in 100\%, in non-specified disproportion in 93\%, in AS in 43\%, in COA in $8 \%$ and in LSVC-CS in 4\%. 80 (61\%) children were born, 6 of them died. The size of LV was normal post-natally in $100 \%$ fetuses with LSVC-CS, in $95 \%$ fetuses with COA and in $68 \%$ fetuses with AS.

Conclusion: There was normal development of the LV in fetuses with pre-natally diagnosed LSVC-CS and COA (small number of termination of pregnancy and low mortality after birth). In AS there was good development of LV after birth in the majority of fetuses, but there was high number of termination of pregnancy (43\%). There were associated abnormalities in the majority of fetuses with non-specified disproportion and none of them is alive.

\author{
Abstract no: I 5 I5 \\ Transcatheter closure of an aorto-cameral fistula with a vascular plug using a retrograde approach: \\ A case report

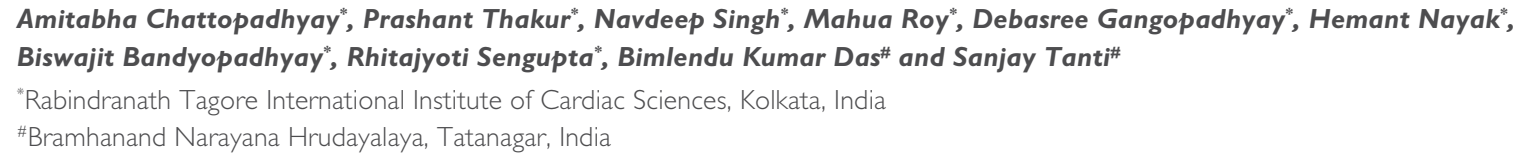

Amitabha Chattopadhyay", Prashant Thakur*, Navdeep Singh*, Mahua Roy*, Debasree Gangopadhyay*, Hemant Nayak*, Biswajit Bandyopadhyay", Rhitajyoti Sengupta*, Bimlendu Kumar Das\# and Sanjay Tanti\#

"Rabindranath Tagore International Institute of Cardiac Sciences, Kolkata, India

\#Bramhanand Narayana Hrudayalaya, Tatanagar, India

Background: Aorto-cameral fistula is a rare cardiac anomaly. Few reports in the medical literature discuss the use of a vascular plug using a retrograde approach to close these fistulae.

Material and method: We describe the case of an 8-year-old girl who presented with complaints of frequent respiratory infections since early infancy, shortness of breath (NYHA class II) and palpitations since the last 2 years. She had a 3/6 continuous murmur at the left lower parasternal area. Echocardiography revealed a huge aorto cameral fistula originating from the right Sinus of Valsalva emptying into the right ventricular (RV) inlet just below the anterior leaflet of the tricuspid valve (TV). The right coronary artery (RCA) was faintly visualised. As the child was symptomatic, she was taken up for a cardiac catheterisation with a goal of possible transcatheter closure of the defect. Aortogram confirmed the echo findings. The right coronary artery (RCA) was faintly visualised originating 8 mm away from the aortic sinus with decreased flow. The narrowest segment of the fistula measured $6.1 \mathrm{~mm}$. There was retrograde filling of the RCA from the left coronary artery (LCA) branches. On temporary balloon occlusion beyond the origin of the RCA with a 6F SwanGanz catheter, no ECG changes were seen. A retrograde approach was used to place a $10 \mathrm{~mm}$ Vascular Plug ( $166 \%$ of the narrowest diameter of the fistula) into the narrowest part of the fistula via a PDA device delivery system resulting in complete occlusion of the fistula. Post procedure RCA angiogram showed normal flow through the RCA with visualisation of the marginal branches which were hitherto not seen due to steal through the fistula. The retrograde approach obviated the need for an arterio-venous loop. No procedural complications occurred with the patient showing better exercise tolerance on follow-up.

Result: Complete occlusion of fistula with improvement in symptoms.

Conclusion: Coronary artery fistula closure by vascular plug through retrograde approach is safe and should be considered as the standard approach.

\title{
Abstract no: 1559
}

\section{Variable approach and outcome of stenting of arterial duct in duct dependent pulmonary circulation}

Nageswara Koneti, Sudeep Verma, V. Karunakar, Shweta Bakhru, Pallavi Kathare, B.R. Jagannath, Tapan Kumar Dash, Sunil Swain, Raghavaraju Penumatsa and Sreenivas Kumar Arramraju

Care Hospital, Hyderabad, India

Background: Stenting of arterial duct has emerged as an alternative for systemic to pulmonary shunt in young infants with duct dependent pulmonary circulation (DDPC). However, the approach of stenting is determined by the anatomy and orientation of the arterial duct. We report our experience of stenting of arterial duct by various approaches and their outcomes.

Materials and methods: Data of 45 (male 3I) young children with DDPC were included for the procedure. Seven children were excluded after angiogram due to unfavourable anatomy in 4 and inability to negotiate wire in 3. Various parameters and outcomes were analysed.

Results: The anatomical diagnosis includes 24 cases of VSD pulmonary atresia, 12 of pulmonary atresia with intact septum and 9 with single ventricle physiology and pulmonary atresia. The median weight was $3 \mathrm{~kg}$ (range $1.7-6.5 \mathrm{~kg}$ ) and age 20 days (range 2 - 370 days). Successful deployment of stent was done in 37 (82\%) children. The approach for stent deployment was determined by the anatomy of the arterial duct and physician experience; femoral artery approach (19), axillary (8), carotid (hybrid) (15) and femoral vein (3) cases. There were 2 deaths; rupture of the ductus in I and the other due to sepsis. There were 3 major procedure-related complications: spasm, dissection and acute thrombosis which were managed. 6 children died at various hospitals during follow-up due to: pneumonia ( I), gastroenteritis with dehydration (I), waiting for surgery (2) and cause could not be detected (2). Successful 2nd stage procedures: systemic-topulmonary shunt (4), Bidirectional Glenn (4) and biventricular repair (4).

Conclusion: Stenting of the arterial duct in DDPC is an alternative palliation using various approaches due to variable anatomy of tortuous vertical arterial ductus. The outcome is determined by the presentation, associated co-morbid condition and underlying complex anatomy. 


\title{
Abstract no: 1570 \\ Usefulness of joint fetal echocardiography and counselling service: Experience in Hong Kong
}

\author{
Shuk Han Lee, Kwok Yin Leung, Teresa Ma and Betty Lau
}

Queen Elizabeth Hospital, Hong Kong

\begin{abstract}
Aim: We collaborated with our obstetricians to run a joint fetal echo and counselling service. This study aims to review the usefulness of this service. Methodology: We reviewed our result from 1993 - 2012. Medical records were retrieved and analysed retrospectively.

Results: 150 pregnant ladies underwent fetal echocardiography in our department. 56 fetuses were found to have cardiac abnormalities from echocardiography and a joint pre-natal counselling service was offered to their families. Detailed anatomy, expected management, prognosis and our life experience of similar cases were explained to the expectant parents. Psychological support was offered as well. Out of the 56 fetuses: 45 had major congenital cardiac abnormalities; only I 3 (29\%) of the pregnancies were electively terminated; I aborted spontaneously; and the rest were born at or near full term.

Follow up: After birth, these infants were re-assessed, stabilised and referred to our neonatal cardiac centre for surgery. The pre-natal diagnoses were accurate in $98.7 \%$ of cases. The transfer and pre-operative course was smooth as appropriate stabilisation was instituted soon after birth. The survival rate after surgery is best in the right ventricular outflow tract obstruction and transposition of great artery groups, amounting to $100 \%$. The oldest child is 13 years of age now. However, all died after surgery for truncus arteriosus, pulmonary artery sling and hypoplastic left heart syndrome, though the number was very small. $60 \%$ with complex heart lesions including heterotaxy syndrome survived with or without surgery. The only fetus diagnosed with premature closure of ductus survived after prompt delivery and neonatal intensive care.
\end{abstract}

Conclusions: In our experience, Joint Fetal Echocardiography and Counselling service is invaluable for the management of fetuses with cardiac abnormalities. It does not only result in a rather low elective abortion rate, but also improves the infants' survival and parents' acceptance after birth.

\author{
Abstract no: I59 I \\ Experience with the Cook Formula stent in paediatric cardiac interventions \\ Oliver Stumper, Bharat Ramchandhani, Patrick Noonan, Vinay Bhole, Chetan Mehta and Rami Dhillon \\ Birmingham Children's Hospital, Steelhouse Lane, Birmingham, United Kingdom
}

Introduction: Balloon expandable stents are an integral part in the catheter treatment of CHD. In the growing child, stents have to be dilatable to greater diameters over time. All current stent designs have limitations. The pre-mounted Cook Formula stent is a recent 316 stainless steel open-cell design licensed for peripheral vascular work.

Methods: Extensive ex-vivo studies were carried out to better understand the stent behaviour regarding shortening and ability to over-dilate the stent. Subsequently 30 stents were implanted in 29 children (median age 0.96 (0.03 - 9.8) years; median weight 7.7 (3.8 - 43 ) kg).

Results: Stents were implanted in the RV outflow tract in I I patients with Fallot-type lesions, in 9 for branch pulmonary artery stenosis ( 3 post Fontan), 4 conduit stenosis, 2 Fontan fenestrations, one each SVC, CoA, PDA.

Stent delivery up to $7 \mathrm{~mm}$ was over a 0.014 " wire via a 4F sheath or 6F guide catheter. 8 or I0mm stents (from 3/2012) were placed over a 0.035" wire using a 7F Mullins sheath. Stent tracking and delivery was excellent. There was no stent shortening for dilatation to nominal diameter and beyond. This allowed for precise placement, avoiding protrusion into adjacent vessels. 16 stents were primarily or subsequently over-dilated without any shortening. The $5 \mathrm{~mm}$ stent could be dilated to $10 \mathrm{~mm}$, and the $10 \mathrm{~mm}$ stent could be dilated to $17 \mathrm{~mm}$ without shortening. There was one circumferential balloon fracture requiring retrieval, and one stent slipped and was removed.

Conclusion: The Cook Formula stent is a versatile pre-mounted balloon-expandable stent that can be significantly over-dilated with virtually no shortening. It is a great addition to the range of stents for use in the catheter treatment of complex CHD in children.

\section{Abstract no: 1599 \\ Management of dextrocardia, situs inversus totalis, mixed type TAPVC, complete AV canal defect, common atrium, bilateral SVC, PDA and severe PAH}

\author{
Sivasubramanian Muthukumar, Charles Jebaraj, Ranjith Karthekayan, Dheeraj Reddy and Periyasamy Thangavelu \\ Sri Ramachandra University, Chennai, India
}

Objective: To present the management dextrocardia, situs inversus totalis, mixed type TAPVC, complete AV Canal Defect, common atrium, bilateral SVC, PDA and severe PAH.

Diagnosis: A 9-month-old baby girl was admitted with failure to thrive and respiratory distress. ECHO evaluation revealed the above-mentioned diagnosis. After pre-operative evaluation the baby was subjected to surgery. Operative finding included mixed type TAPVC: right superior and middle pulmonary veins and left inferior pulmonary vein draining into common venous chamber; vertical vein from the common chamber in RSVC; left superior vein to LSVC; right upper lobe vein to RSVC; morphologic RA was on right side receiving RSVC and hepatic vein; morphologic LA on the left side receiving LSVC and IVC; and unroofed coronary sinus. Complete AV canal defect: Rastelli Type A with large inlet VSD, severe MR - AML cleft, common atrium.

Result: The procedure included TAPVC repair and AV canal repair. Rerouting of pulmonary veins with wide anastomosis between the common venous chamber and $\mathrm{mLA}$. Vertical was ligated. Since a in nominate vein was present, LSVC was interrupted and left superior vein diverted to $\mathrm{mLA}$. Regarding AV canal repair, a double patch technique was used. VSD closure, MV repair with AML cleft repair, Tricuspid valve repair, ASD closure with pericardia patch creating an atrial baffle diverting IVC, RSVC and hepatic vein to mRA. Core cooling done to $28^{\circ} \mathrm{C}$. Total CPB time was 325 minutes and aortic cross-clamp time 235 minutes. Total ventilatory support for 86 hours and inotropes for 6 days. ICU stays till 8th POD.

Conclusion: Baby was discharged on 16 th POD with stable haemodynamics and normal oral feeds. 


\section{Abstract no: 1601 \\ Stenting of bilateral arterial ducts in complex congenital heart disease}

\section{Nawal AlAbdulkarim, Khalaf AIKhalaf and Atif AISahari}

Prince Sultan Cardiac Centre, Riyadh, Saudi Arabia

Background: Maintaining ductal patency in duct-dependent congenital heart lesions by implantation of coronary stents is an alternative to systemic pulmonary shunt in selected cases and lesions with suitable anatomy. Bilateral arterial ducts are a very rare pattern of pulmonary blood flow in congenital heart disease with pulmonary atresia with non-confluent pulmonary arteries. Ductal closure lead to severe systemic hypoxia indicating emergent surgical palliation or repair making percutaneous arterial duct stenting a possible alternative to surgery in such high risk patients.

Methods: We describe 2 critical neonates with complex heart disease and discontinuous pulmonary arteries surviving on bilateral arterial ducts who successfully underwent transcatheter ductal stenting as first-step palliation toward lower risk surgery.

Results: The 2 patients are summarised in the Table:

\begin{tabular}{|c|c|c|c|c|c|}
\hline Patient & Age (Weeks) & Weight (kg) & Sex & Anatomy & Procedure \\
\hline Number I & $\begin{array}{c}4 \\
\text { (Referred at } \\
4 \text { weeks of age) }\end{array}$ & 3.8 & Male & $\begin{array}{l}\text { Single double inlet left } \\
\text { ventricle, atretic right } \\
\text { atrioventricular valve, } \\
\text { restrictive ASD, pulmonary } \\
\text { atresia, disconnected } \\
\text { BPAs supplied by } \\
\text { bilateral ducts }\end{array}$ & $\begin{array}{c}\text { Atrial septostomy/ } \\
\text { bilateral coronary stent } \\
\text { placement in each ductus }\end{array}$ \\
\hline Number 2 & $\begin{array}{l}8 \\
\text { (diagnosed ante-natally } \\
\text { then delayed to complete } \\
\text { fungal sepsis treatment) }\end{array}$ & 4.3 & Male & $\begin{array}{l}\text { Single ventricle, atretic } \\
\text { left atrioventricular valve, } \\
\text { pulmonary atresia, right } \\
\text { arch disconnected BPAs } \\
\text { supplied by bilateral ducts }\end{array}$ & $\begin{array}{l}\text { Bilateral coronary stent } \\
\text { placement in each ductus }\end{array}$ \\
\hline
\end{tabular}

Ductal stabilisation was achieved with coronary stents: Patient number I is awaiting first palliative surgical procedure; Patient number 2 underwent a successful first palliative surgical procedure, and continues to do well in clinical follow-up at I 4 months of age.

Conclusions: Stent implantation is a technically feasible, safe and effective palliative option in high risk surgical patients with bilateral arterial ducts with pulmonary atresia and non-confluent pulmonary arteries.

\section{Abstract no: 1610}

\section{Early experience with ductal stenting in a public-sector service in South Africa}

\section{Paul Adams, Gcina Dumani, Barend Fourie and Antoinette Cilliers}

Division of Paediatric Cardiology, Chris Hani Baragwanath Academic Hospital, University of Witwatersrand, Johannesburg, South Africa

Background: Stenting of the ductus arteriosus has become a viable alternative to surgical shunts for selected patients with duct dependant pulmonary blood flow. From February 2012, we began stenting the arterial duct in order to provide an alternative treatment option for these infants.

Materials and methods: Clinical presentation, procedural and patient outcome data were extracted from patient files and clinic database. To be eligible for potential stenting, patients had to have duct dependant pulmonary blood flow, no left pulmonary artery stenosis and not accepted immediately for a surgical shunt. We will compare the outcomes of these patients with the 26 other patients who had similar diagnoses made since January 20 I0, prior to the procedure being available.

Results: Four patients with duct dependant pulmonary blood had stenting of their ductus arteriosus from early 2012 onwards. Two patients had pulmonary atresia and intact septum, another had pulmonary atresia with a small ventricular septal defect, and the fourth had tricuspid atresia IA. All procedures were successful, however I patient required a repeat catheterisation as arterial access could not be obtained at first procedure. After observation and stabilisation, patients were transported to their referral hospitals and then discharged home. Outcomes of the other 26 patients are being summarised presently.

Conclusions: Stenting the ductus arteriosus is not a technically simple procedure. Our early experience suggests it can be performed safely and effectively in our setting. This would offer an alternative to surgical shunts, where the more-invasive surgical procedure is often delayed due to personnel and infrastructure availability constraints, or is deemed too high risk. Building experience in these techniques is critical, not only beneficial for the patient undergoing the procedure but could relieve some of the burden from the surgical waiting lists. 


\section{Abstract no: 1630 \\ Temporary use of small stents in critical congenital heart disease}

\section{Stephen Brown, Carri-Lee Greig, Daniel Buys and Michael Long}

Department of Paediatric Cardiology, University of the Free State, Bloemfontein, South Africa

Background: Surgery in children with critical congenital heart disease is associated with a high morbidity and mortality. The aim of this study was to look at the short and medium term outcomes of stent implantation in these patients and to determine whether surgery could be delayed in a developing country.

Materials and methods: Eighteen children were included in a retrospective review from October 2005 - May 20 I2. Vascular access was obtained via the femoral artery and/or vein. Balloon ex pandable coronary bare metal stents were used. Follow-up included clinical assessment and echocardiography.

Results: Eighteen cases were successfully stented. Indications were: aortic arch obstruction ( $n=9)$; right ventricular outflow tract obstruction ( $n=4)$; systemic-topulmonary artery shunt stenosis $(n=3)$; pulmonary venous chamber stenosis $(n=1)$; and PDA stenting to preserve a discontinuous left pulmonary artery $(n=1)$. 8 patients had residual problems following prior surgery. Median age was 4.8 months (range: 0.1 - 65.1) and median weight 4.6kg (range: $1.7-17)$ ). Number of stents used: I $(n=12), 2(n=5)$ and $4(n=1)$. Implanted stent diameters: $2.5 \mathrm{~mm}(n=2) ; 3.5 \mathrm{~mm}(n=2) ; 4.0 \mathrm{~mm}(n=4) ; 4.5 \mathrm{~mm}(n=3) ; 5.0 \mathrm{~mm}(n=9) ; 5.5 \mathrm{~mm}(n=1)$; $6.0 \mathrm{~mm}(n=3)$ and $8.0 \mathrm{~mm}(n=2)$. Paired pressures were not obtainable in all cases as most patients were critically ill. Saturations improved from a median 83.5 $-90 \%$ in patients with obstructive pulmonary blood flow $(n=4)$. Peri-procedural complications included temporary rhythm disturbances $(n=3)$. There was no periprocedural mortality. Last follow-up was at a median 200 days (range: 10 - 1406). A 2nd stent procedure was performed in 6 patients ranging between 3.8 and 17.8 months post initial stent placement. 5 cases proceeded to surgery. 5 cases demised at a median of 73 days (range: 20 - 422), all at home secondary to unknown causes. Three patients have been lost to follow-up. Five patients remain in follow-up.

Conclusions: Mortality in this high risk group remains high. Stenting is feasible and may delay surgery in some however, problems in developing countries present unique challenges to patient follow-up.

\section{Abstract no: 1634 \\ Stenting of pulmonary arteries and conduits: An effective way of palliation in congenital heart diseases with narrowed pulmonary arteries}

Sivakumar Kothandam, Sreeja Pavithran, Anilkumar Singhi and Anpon Bhagyavathy

MIOT Hospital, Chennai, India

Background: Pulmonary artery and conduit stenosis lead to morbidity following conotruncal defect surgery. They may rarely occur denovo in some syndromes. We present our 5 year experience in pulmonary artery (PA) stenting to rehabilitate narrowed pulmonary arteries and conduits.

Methods: Thirty three consecutive patients who underwent PA stenting in a single centre from 2007 - 201 I were reviewed and their follow up analysed.

Results: The cohort of 33 patients had 16 males; age ranged 2 months to 38 years (median 7 years) and weight ranged $2 \mathrm{~kg}$ - $70 \mathrm{~kg}$ (median $25 \mathrm{~kg}$ ). Forty seven balloon expandable stents were implanted. All except 2 had previous cardiac surgery and most followed surgical repair of Tetralogy of Fallot. The sites stented were left PA in II patients, right PA in 2, bilateral PA in II, calcified homograft and xenograft conduits in 7, native pulmonary annulus in I and supravalvar main PA in I patient. The mean stenotic gradient decreased from $65 \mathrm{mmHg}-14 \mathrm{mmHg}$. The ratio of right ventricle to aortic systolic pressure reduced from 0.9 - 0.4 . Four patients had other interventions performed during the procedure. There was no mortality. One patient with Cutis Laxa and bilateral PA stenosis had hyperperfusion pulmonary hemorrhage in the right lung following the procedure. Stent migration was noted in 4 patients which needed use of another stent. No patient needed urgent surgical procedures for stent related complications. The median follow up period was 25 months. All the patients were asymptomatic and none of the patients required reintervention on follow-up.

Conclusion: PA Stenting provides effective interim palliation in native and postoperative pulmonary artery stenosis. Even in less than ideal situations like a calcified and stenosed right ventricular outflow tract conduit, the procedure prolongs the lifespan of the conduit and delays conduit replacement.

\section{Abstract no: 1695 \\ Aneurysm of the foramen ovale flap and ventricular size discrepancy in pre-natal echocardiography \\ Anna Lamberti, Mauricio Guirgis, Carine Vastel, Ronan Bonnefoy, Isabelle Denjoy, Jean Marc Lupoglazoff and Constance Beyler Robert Debré Hospital, Paris, France}

Background: Ventricular size discrepancy (VD) is easily detected and, in routine general fetal echography, echocardiography is therefore performed with the aim to prenatally detect left ventricle inflow or outflow obstructive lesions and aortic arch anomalies. However VD can be a physiological aspect late in pregnancy or associated to anomalies such as left vena cava or aneurysm of the foramen ovalis flap (AFOF).

Materials: Among 3500 pre-natal echocardiographies performed in our unit from January 2008 - July 20I2, 45 AFOF were detected with variable degree of VD late in pregnancy: mean term was $33.5 \pm 0.7$ weeks of gestation $(\mathrm{w})$. In all patients ventricular arterial ratio: right/left ventricular diameter (VR), aorta/pulmonary artery diameter (AR) and aortic isthmus diameter (AID) were measured, colour Doppler images and velocities were recorded. In most cases analysis of the aortic arch showed a complete aortic arch with normal flow velocity and direction; AID was considered as normal when $>3 \mathrm{~mm}$ after the 30 th week or $0.1 \mathrm{~mm} \times \mathrm{w}$ when earlier in gestation.

Results: Group I (GI) 33 patients: VR<1.5: 3 I =normal AID (3I), small AID (2); Group 2 (G2) I2 patients: VR I.5=normal AID (8), small AID (4). Only I5\% of the 45 patients with AFOF and VD had AID suspicious for post-natal coarctation. In these few cases birth was planned in our hospital with intensive care and paediatric cardiology units.

Conclusion: AFOF is frequently associated with VD in pre-natal echocardiography. If the AID is normal with forward flow and normal velocity, AFOF can be considered as the main factor responsible for $\mathrm{VD}$ and there is low risk of post-natal coarctation. This screening is useful to plan in utero transfers from peripheral hospitals and avoid non-productive decisions. 


\title{
Abstract no: 1697 \\ Screening of fetal cardiac disease in Saudi Arabia: A tertiary cardiac care centre experience
}

\section{Nawal AlAbdulkarim}

Prince Sultan Cardiac Centre, Riyadh, Saudi Arabia

Introduction: Antenatal detection of cardiac malformations remains suboptimal in most areas of the world with a gap in diagnosing serious cases of congenital heart disease.

Method: To describe the current status and factors affecting detection of congenital heart disease (CHD) ante-natally in Saudi Arabia.

Results: Fetal Echocardiography/Cardiology started in 1980s at our institution but the rate of detection of fetal CHD remains low with almost half the cases referred with serious CHD not detected in antenatal screening. Contributing factors include high incidence of CHD in low risk pregnancies, a sub-optimum referral system, lack of regional/national fetal cardiac screening programmes and the need for more countrywide training for cardiac screening at obstetric units, Conclusions: The overall rate of detection of major congenital heart disease before birth remains low. Cases will only be detected by screening of the low-risk population at the time of routine obstetric scanning. A common effective practice standard to detect CHD ante-natally need to be established nationally/ regionally and throughout the world. It ought to offer enhanced training and education on screening of the fetal heart plus an appropriate referral/ networking system.

\section{Abstract no: $|7| \mid$}

Bacterial endocarditis after transcatheter pulmonary valve replacement using the Melody Valve

\author{
Evan Zahn*, Lee Benson", Andreas Eicken ${ }^{\dagger}$, Jacqueline Kreutzer ${ }^{\ddagger}$ and Doff McElhinney \\ "Cedars-Sinai Medical Center, Los Angeles, California, United States of America \\ \#The Hospital for Sick Children, Toronto, Ontario, Canada \\ †German Heart Centre Munich, Munich, Germany \\ *Children's Hospital of Pittsburgh of UPMC, Pittsburgh, United States of America \\ §New York University Langone Medical Center, New York, United States of America
}

Background: Transcatheter pulmonary valve (TPV) Implantation has emerged as a viable therapy for treatment of right-ventricle-pulmonary artery conduit dysfunction. Little is known about the incidence, clinical course, and outcome of bacterial endocarditis (BE) following TPV implantation.

Methods: The datasets of 3 ongoing prospective multicenter trials (US IDE Continuation, European/Canadian Post-Market Surveillance, and US Post-Approval Studies) were queried for cases of BE following TPV implantation. Any clinical episode characterised as definite or presumed endocarditis with confirmatory blood cultures, regardless of TPV involvement was considered BE and did not require satisfaction of published criteria. Cases were characterised as TPV-related (TPV-R) or not TPV-R based upon echocardiographic evidence of TPV vegetations and/or new/progressive dysfunction.

Results: The study population included 31I TPV patients with 687. One patient-years of follow-up (median 2.5 years, max 5.1 years) of whom I6 were diagnosed with BE (first episode rate: $2.4 \%$ per patient-year). Median duration from implant to BE was I.3 years (50 days - 4.7 years). Six patients had TPV-R BE, 4 with vegetations, 3 with TPV dysfunction. For the TPV-R group, the annualised rate of a first BE episode was $0.88 \%$ per patient-year, and freedom from BE was $97 \pm 1 \%$ at 4 years. Vegetations were visualised in non-TPV locations in 4 patients. All 16 patients were treated with IV antibiotics, 4 underwent explantation ( 3 with TPV-R BE), and 2 received a second TPV. There were 2 sepsis-related deaths and 2 patients developed a second episode of endocarditis. Data regarding adherence to recommended BE prophylaxis were not available.

Conclusion: BE has occurred in all 3 prospective multicenter studies in North America and Europe. Most cases were subacute or chronic, did not involve the TPV, and responded to antibiotic treatment. More data are necessary to understand specific risk factors in this population.

\section{Abstract no: 1725 \\ A Population-based study on transcatheter interventions for congenital heart disease in children and adults during 22 years}

\author{
Signe Holm Larsen",\#, Kristian Emmertsen*, Vibeke Elisabeth Hjortdal\# and Jens Erik Nielsen-Kudsk* \\ "Department of Cardiology, Aarhus University Hospital, Skejby, Denmark \\ \#Department of Cardiothoracic Surgery, Aarhus University Hospital, Skejby, Denmark
}

Background: Advances in knowledge and interventional technology have increased the use of transcatheter interventions for congenital heart disease. We report experience from a single center providing treatment of children and adults from a background population of 3 million.

Materials and methods: Patients were identified using a clinical database on cardiac interventions from January I 990 - December 201 I. Linear regression was used to test for association between number of procedures and time, logistic regression for association between complications and time.

Results: A total of I 760 procedures were performed in I 489 patients (777 females, median age 5.1 years, 5 - 95 th percentile 0.0 - 59.5 years): closure of patent arterial duct $(n=406)$, closure of atrial septal defect $(n=305)$, pulmonary valvotomy $(n=203)$, dilatation/stent treatment of coarctation of the aortae ( $n=\mid 68)$, atrial septostomy $(n=148)$, dilatation/stent/percutaneous valve in the pulmonary artery, braches and conduits ( $n=\mid 34$ ), aortic valvotomy ( $n=9 \mid$ ), closure of patent foramen ovale $(n=86)$, procedure in systemic vein or pathway $(n=52)$, procedures with surgical shunts $(n=12)$, closure of ventricular septal defects $(n=8)$, and miscellaneous $(n=73)$. Over the years there was a fifth-fold increase in number of procedures $(p<0.00 \mathrm{I})$. Similar catheterization techniques were used for some acquired diseases such as post infarction ventricular septal defects, postsurgical or malignant vessel obstruction etc. $(n=100)$.

Complications in hospital were 224 (12.7\%), 45 (2.6\%) of these were considered major (death, life-threatening events requiring immediate therapy, need for 
surgical intervention, significant unanticipated permanent impairment resulting from catheterization). Of the major complications 16 (36.6\%) were arrhythmias treated successfully during the procedure, without sequelae. There were 10 deaths $(0.6 \%)$, none after 2006. The rate of complications decreased during the study period $(p<0.001)$.

Conclusion: In this population of patients with congenital heart disease treated with transcatheter intervention, there was an increase in the number of procedures performed during the study period and a decrease in complication rate.

\author{
Abstract no: 1770 \\ Prenatal diagnosis of Ebstein's anomaly: Report of 4 cases \\ Ana Flavia Torbey", \#, Maria de Fatima Leite" \\ Jose Pedro Silva and Fabio Bergman" \\ "Universidade Federal Fluminense, Brazil \\ \#Baby Cor Cardiologia Paediatrica e Fetal, Rio de Janeiro, Brazil \\ tInstituto Fernandes Figueira, Rio de Janeiro, Brazil \\ ¥Hospital Servidores do Estado, Rio de Janeiro, Brazil \\ §perinatal, Rio de Janeiro, Brazil \\ Beneficencia Portuguesa de Sao Paulo, Sao Paulo, Brazil
}

Ebstein's anomaly (EA) is a rare malformation of tricuspid valve leaflets and can be detected in fetal life by important cardiomegaly during obstetric ultrasonographic examination, the most common indication of fetal echocardiogram. We describe the evolution of four cases of fetal diagnosis of EA

Four cases of prenatal diagnosis of EA, from 3 units of Fetal and Pediatric Cardiology of Rio de Janeiro are reported. Patients were referred to fetal echocardiogram due to cardiomegaly observed during obstetric assessment, between 25 and 37 wga. All fetuses had significant tricuspid regurgitation, and significantly increased right atrium. Ventricular septal tricuspid leaflet was attached to ventricular septum, obstructing right ventricular outflow tract with functional pulmonary atresia (PA) in two cases, and true pulmonary atresia, associated with pulmonary branches hypoplasia in the other two fetuses. One fetus had heart failure with periods of atrial tachycardia treated with oral maternal digitalis. Two patients died, one on the 38th gestational week due to corioaminionitis confirmed by anatomopathologic study after birth and another due to pulmonary hypoplasia. Another case of PA needed a systemic-pulmonary shunt during neonatal period and had a tricuspid valve repair one month after birth with good postoperative evolution, although he developed tachyarrhythmia that was controlled with amiodarone. The last one had functional PA but pulmonary blood flow became normal, after measures to low pulmonary vascular resistance.

Fetal echocardiogram is an important tool for the diagnosis of congenital heart disease with severe manifestations and complications during the perinatal period. It permits the medical team to create strategies to prevent the dangers of metabolic acidosis and hypoxemia in the first hours after birth. The presence of intrauterine pulmonary hypoplasia, severe tricuspid regurgitation and pericardial effusion are signs of poor prognosis.

\title{
Abstract no: 1775 \\ Percutaneous transmitral commissurotomy in children: Intermediate term results with special reference to pulmonary hypertension
}

\section{Najma Patel}

National Institute of Cardiovascular Disease, Karachi, Pakistan

Objective: To know the long term results of PTMC and fate of pulmonary hypertension in children undergone PTMC.

Material: During last I I years 138 children age range 10.48 2 2.24 years $(3.5$ - 16) had PTMC. Indications were echocardiographic evidence of moderate to severe MS plus PAH $>50 \mathrm{mmHg}$ and/or FC III-IV.

Results: Pre PTMC MPG across mitral valve (MV) was $18.6 \mathrm{mmHg} \pm 3.4$ which decreased to $7.6 \mathrm{mmHg} \pm 2.21$. MVA was $0.62 \mathrm{~cm}^{2} \pm 0.12$ which increased to $1.6 \mathrm{~cm}^{2} \pm 0.3$. PAP was $83 \mathrm{mmHg} \pm 13$ decreased to $50 \mathrm{mmHg} \pm 14.5$. 3 were unsuccessful. Two deaths occurred: One within I/2 hour in patients with PAP of $100 \mathrm{mmHg}$ and LV dysfunction had no MR or tamponade; the other died after 24 hours had thromboembolism of LAD, revived and had successful PTMC, but died after 24 hours. Another patient had tamponade, which was drained and had successful PTMC after I week. There was no change in MR in 50 patients: + I (83); +2 (8) and +3 (3). After a mean follow-up period of 44.3 years \pm 30.6 of I I 0 patients ( 10 months - 10 years): 8 needed redo PTMC after $5.37 \pm 2.3$ years (.5-8 years); 2 had MVR after I and 7 years. Systolic PAP was $83 \mathrm{mmHg} \pm 13$ pre procedure which decreased to $50 \mathrm{mmHg} \pm \mid 4.5$ immediately after and to $42 \mathrm{mmHG} \pm 13$ after 6 months. Immediately after PTMC 54 patients had PAP more than 50mmHg, after 6 months 24pts. Out of these 2 died, of which one had severe PAH despite adequate relief of MV who died after 3 years. Another had mild MS and +2 MR with persistent severe PAH and had MVR after I year, PAP did not decline after surgery and died after 2 years of PTMC. Both have initial PAP $>100 \mathrm{mmHg}$.

Conclusion: PTMC is effective in relieving stenosis, however initial high PAP is a predictor of persistent pulmonary hypertension. 


\title{
Abstract no: 1783 \\ Congenital left ventricular aneurysm: Fetal diagnosis
}

\author{
Ana Flavia Torbey*,, Aurea Souza*, Tatiana Berg ${ }^{\dagger}$, Sandra Pereira ${ }^{\dagger}$, Luciana Duarte*, Sandra Possato", Maria de Fatima Leite $\#$, Alan Silva \\ and Fabio Bergman" \\ "Universidade Federal Fluminense, Rio de Janeiro, Brazil \\ \#Baby Cor Cardiologia Paediatrica e Fetal, Rio de Janeiro, Brazil \\ tHospital Servidores do Estado, Rio de Janeiro, Brazil \\ \#Instituto Fernandes Figueira, Rio de Janeiro, Brazil
}

Background: Congenital left ventricular aneurysm (CLVA) is a rare cardiac malformation occurring in 0.5: I 00000 births, regardless of gender and presents as a single lesion in $75 \%$ of cases. Most patients are asymptomatic, although some complications such as congestive heart failure, arrhythmias, thromboembolism and ventricular wall rupture may occur.

Material and method: A 29-year-old primigravida without risk factors was referred to fetal echocardiography at 33 weeks of gestational age (wga) with diagnosis of cardiomegaly during obstetric ultrasound. Fetal echocardiogram showed a $1.9 \mathrm{~cm}$ expansive rounded area in left ventricle apex, consistent with a CLVA with slow velocity flow inside. Doppler analysis detected supraventricular extra systoles and bigeminism periods. No other structural changes, thrombi or signs of heart failure were observed during subsequent evaluations.

Results: Delivery occurred at term without complications and post-natal echocardiogram confirmed the diagnosis. During the Ist weeks of life the newborn developed supraventricular tachycardia which is successfully controlled with beta blockers. Since then she has remained asymptomatic.

Conclusions: Etiology of CLVA is unknown and may occur by localised weakening of the ventricular wall during embryogenesis, infection or ischaemia of the ventricular myocardium. It can be produced by stenosis, hypoplasia or abnormal development of the coronary arteries. Fetal echocardiogram is essential for early diagnosis as well as monitoring during pregnancy and birth to detect complications such as rupture of the wall, thromboembolism, arrhythmias and heart failure, whereas the latter can receive intra-uterine treatment. The natural history of CLVA is not well studied so the approach must be individualised for each case.

\author{
Abstract no: 1789 \\ Trends in prenatal diagnosis of transposition of the great arteries with intact ventricular septum and \\ impact on outcomes \\ Maria Clara Escobar*, Alejandra Bueno-Martinez*, Brian Kalish", Ravi Thiagarajan*, David Brown*, Sitaram Emani*, Pedro Del Nido* \\ and Wayne Tworetzky* \\ "Boston Children's Hospital, Boston, Massachusetts, United States of America \\ \#Harvard Medical School, Boston, Massachusetts, United States of America
}

Background: In transposition of the great arteries with intact ventricular septum (TGA/IVS), changes occur after birth that, if left untreated, could lead to haemodynamic compromise and death. Pre-natal diagnosis (Dx) allows for delivery site planning and timely neonatal management. Despite TGA being a major heart defect, detection is challenging for obstetricians.

Objective: To evaluate temporal trends in pre-natal Dx and its impact on neonatal morbidity and mortality.

Methods: Patients with TGA/IVS referred for surgical management between 1995 and 2012 were included. The study time was divided into 3 6-year periods. We compared variables pre-op and post-op between those diagnosed pre- or post- natally. Variables included gestational age and weight at birth, age on admission, mechanical ventilation, ECMO, metabolic acidosis, timing of septostomy and surgery, ICU and hospital stay, and mortality.

Results: Of the 295 patients, 78(26\%) had prenatal Dx. There was an increase in the pre-natal diagnosis over the years from I0\% initially to $42 \%$ over the last few years. Gestatonal age and birth weight was similar between groups. Age at admission ( I.4 vs. 0), at septostomy ( 1.2 vs. 0.3 ) and surgery (4.7 vs. 3.9) were greater in post-natal Dx group. The post-natal Dx group had more metabolic acidosis (19\% vs. 7\%), mechanical ventilation (7I\% vs. 40\%) and need for ECMO (3.7\% vs. I.3\%) prior to surgery. The overall mortality was $2.7 \%$. In the post-natal Dx group $7 / 2$ I7 patients died, 3 pre-op and 4 post-op. There was I death (pre-op) in the pre-natal Dx group. Hospital and ICU stay was not significantly different between groups.

Conclusions: Pre-natal detection rate of TGA/IVS increased significantly over the study period, but is still disappointingly low at <50\%. Although the mortality rate did not differ between pre- and post-natal Dx groups, patients with pre-natal Dx had significantly less metabolic acidosis, earlier admission and septostomy compared to those diagnosed post-natally.

\section{Abstract no: 1798 \\ Fetal cardiac masses with large pericardial effusion: A case report}

Ana Flavia Torbey", \#, Aurea Grippa",\#, Sandra Pereira ${ }^{\dagger}$, Andrea Avilla ${ }^{\dagger}$, Martha Vilela ${ }^{\dagger}$, Lilian Stwert", Carla Kluft', Fernanda Balzana ${ }^{\dagger}$ and Maria de Fatima Leite" $\$$

"Universidade Federal Fluminense, Rio de Janeiro, Brazil

"Baby Cor Cardiologia Paediatrica e Fetal, Rio de Janeiro, Brazil

tHospital Servidores do Estado, Rio de Janeiro, Brazil

*Instituto de Cardiologia Aloisio de Castro, Rio de Janeiro, Brazil

§Instituto Fernandes Figueira, Rio de Janeiro, Brazil

Background: Primary tumours of the heart are rare with a prevalence rate ranging from I: I 000 - I: 100000 of the general population autopsy studies. Most of all, primary cardiac tumours are benign and rhabdomyoma is the most common in paediatric population.

Methods and material: A 16-year-old pregnant teenager at the 37th week was referenced to the fetal echocardiography evaluation. A large pericardial effusion was detected with partial right atrium collapse and a great number of myocardium well-delimitated masses distributed in the right and left ventricle walls and left atrium posterior wall without outflow obstruction. 
Results: Magnetic resonance images were performed to study the heart tumours distribution and screening to search for extra cardiac anomalies confirming the tuberous sclerosis complex diagnosis. Restrictive pericardial effusion was observed post-natally and the peri-cardiocentesis was performed. A new effusion was observed but the newborn evolved a severe sepsis and died on the 7th day of life. Unfortunately neither an autopsy nor even a cytological study of the pericardium effusion was performed. The teenage was referred for paediatric ambulatory attendance because she also had a diagnosis for tuberous sclerosis. Conclusions: Fetal echocardiography can provide an accurate diagnosis for the theoretical rhabdomyoma diagnosis and the necessary follow-up considering the disorder's morbidity. Serious complications can occur such as cardiac arrhythmia, flow obstruction with low cardiac output, hydrops fetalis and sudden death. Knowledge of the outcome of affected fetuses and associated tuberous sclerosis complex with cardiac rhabdomyoma is necessary for adequate pre-natal counselling and the planning of pre-natal and/or post-natal treatment.

\title{
Abstract no: 1804 \\ Referral patterns for fetal echocardiography and impact of prenatal diagnosis of congenital heart disease at a tertiary care centre in Greece
}

\author{
Maria Kiaffas", Cleo Laskari", Ariadne Malamitsi-Puchner", Athanasios Gouliamos', Eystathios Iliodromitis ${ }^{\ddagger}$ and Spyridon Rammos" \\ "Department of Paediatric Cardiology, Onassis Cardiac Surgery Centre, Kallithea, Greece \\ \#Department of Neonatology, Aretaieion General Hospital, Athens, Greece \\ tDepartment of Radiology, Aretaieion General Hospital, Athens, Greece

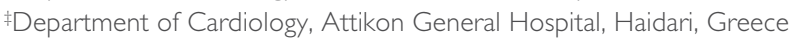

Objective: The aim of this study is to follow the patterns of referral for fetal echocardiograms (FE) and the impact of the pre-natal diagnosis of congenital heart disease (CHD) at a tertiary paediatric cardiology/cardiac surgery care centre in Greece.

Methods: This is a retrospective study; all fetal echocardiograms performed from 2005 - 201 I at our centre were reviewed. The main indication for the study, and the positive diagnoses of CHD were documented and analysed.

Results: 4694 FE studies were performed between 2005 and 201 I in 4120 patients at our centre. The gestational age (GA) at which the examination was performed ranged from 16 - 37 weeks. There was a gradual rise in the annual number of studies with the rate of detection of significant CHD being almost constant. There was a gradual increase in the number of patients being referred due to findings during the Ist trimester fetal scan: increased nuchal translucency, tricuspid regurgitation and flow reversal at the ductus venosus. Referral rate for suspected CHD at the anomaly scan, positive family history of CHD, arrhythmias, maternal diabetes and treatment with medications as well as suspected chromosomal anomalies remained important factors for cardiac evaluation of the fetus by a specialist. A total of 392 cases of significant CHD were diagnosed and 145 cases opted for termination of pregnancy.

Conclusions: This is a large series of FE in a small country's population rather homogeneous regarding racial, cultural and religious aspects. Our study over the last few years suggests that Ist trimester findings in the fetus influence the referral patterns for FE compared to known pre-existing indications, and that the GA for initial cardiac evaluation by a specialist is gradually decreasing. Termination of pregnancy is also the preferred option for significant CHD in our country despite the availability of appropriate post-natal interventional or surgical treatment.

\section{Abstract no: 1805 \\ Maternal drug levels and response time to maternal anti-arrhythmic treatment in fetal tachyarrhythmia}

Orhan Uzun, Kadir Babaoglu, Yusuf I. Ayhan, Katie L. Hardingham, Anju Sinha and Bryan Beattie

Department of Fetal Medicine, Cardiff, Wales, United Kingdom

Background: Fetal tachycardia is an emergency and requires rapid evaluation of fetal rhythm and prompt initiation of anti-arrhythmic medication. Fetal mortality is unacceptably high even in treated cases. Fetal response time and maternal anti-arrhythmic drug levels may play an important role in unfavourable outcomes. Methods: A 10 year review of fetuses presenting with tachyarrhythmia at a tertiary institution. A combination of flecainide and digoxin was the preferred treatment. Maternal anti-arrhythmic levels and fetal response time to tachycardia resolution have been evaluated.

Results: There were 44 patients and 37 fetuses were given flecainide and digoxin combination treatment. The sinus rhythm was restored in mean of 4.85 days (range, I - 14 days) in fetuses with supraventricular tachycardia (96\%). The fetal response time in atrial flutter was slightly longer with a mean of I0 days (range, I - 18 days) but all hydropic fetuses had complete resolution of ascites, pleural, and pericardial effusions with rate control. Resolution of hydrops took as long as 2 weeks after normalisation or reduction of fetal heart rate below $160 \mathrm{bpm}$. There was no correlation between maternal drug levels and maternal electrocardiographic parameters or fetal response time to tachycardia.

Conclusion: Combination treatment is well tolerated by pregnant women, and there was no major side effect requiring withdrawal of anti-arrhythmic medication. The 2-drug combination was effective in improving fetal haemodynamics in most cases, but fetal response time showed no significant correlation with maternal drug levels.

\footnotetext{
Abstract no: 1824

Transcatheter occlusion of large congenital coronary cameral fistulae using the Amplatzer vascular plug II

Farirai Fani Takawira and Greenwood Sinyangwe

Steve Biko Academic Hospital and the University of Pretoria, Gauteng, South Africa

Background: A coronary cameral fistula is a rare connection between a coronary artery and a cardiac chamber or vein. Most of these fistulae have a congenital origin; though they can be acquired. Congenital coronary cameral fistulae (CCCF) are rare abnormalities. Their management has ranged from cardiac surgery to transcatheter occlusion using multiple coils, bags, glue and, more recently, the Amplatzer devices. The most recently developed Amplatzer vascular plug II (AVPII), for peripheral vascular occlusions has only been used for the occlusion of coronary cameral fistulae in few published case reports.
} 
Materials and methods: Case I: A 28-year-old woman presented with a history of decreased effort tolerance and episodes of chest pain on exertion. On examination she was in NYHA (cardiac failure) class 2. Her pulse was 75bpm and tended to collapse. All her pulses were palpable. Her BP was I20/50mmHg. A 2/6 continuous murmur was audible in the left lower sternal boarder. Echocardiography demonstrated a large right coronary cameral fistula (CCCF) draining into the right ventricle. At cardiac catheterisation under general anaesthesia, a $14 \mathrm{~mm}$ Amplatzer vascular plug II (AVPII) was selected and deployed into the distal portion of the fistula with successful occlusion of the CCCF. She remains well 2 years after the procedure. Case 2: An 8-year-old boy presented with an incidental murmur found during evaluation for an URTI. He had no cardiac symptoms. On examination he had a pulse of 80/minute and bounding pulses. His BP was II0/45mmHg with a wide pulse pressure. Echocardiography demonstrated a large left CCCF draining into the LV. During cardiac catheterisation the fistula was successfully embolised using the Amplatzer vascular plug II.

Results: Both patients had successful transcatheter occlusion of the coronary cameral fistulae. Since the procedures they have remained well for $>$ I 8 months. No complications were encountered.

Conclusions: We report successful occlusion of large CCCF using the AVPII. The advent of the Amplatzer Vascular Plug (AGA Medical Corporation; Plymouth, MN, USA) for peripheral vascular applications has added another tool in the interventionist's armamentarium for closing large coronary artery fistulae.

\section{Abstract no: 1835}

Sub-pulmonary stenosis assessed in mid-trimester fetuses with Tetralogy of Fallot: A novel method for predicting post-natal pulmonary valve Z-score and surgical management

Elena N. Kwon, Ira Parness, Shubhika Srivastava, James Nielsen and Miwa Geiger

Mount Sinai Medical School, New York, United States of America

Background/hypothesis: The severity of right ventricular outflow tract (ROVT) obstruction impacts post-natal outcomes in Tetralogy of Fallot (ToF). There is no existing data relating to sub pulmonary stenosis (SPS) severity in the 2nd trimester to post-natal ToF course. We hypothesised that quantification of pre-natal SPS in the 2nd trimester would identify infants with smaller pulmonary valves who would require earlier surgery and/or undergo trans-annular patch (TAP) repairs.

Materials and methods: We retrospectively identified fetuses with ToF from 1998 - 2010 diagnosed at $\leq 26$ weeks gestation. Data evaluated included pre-natal and post-natal pulmonary valve Z-scores (PVZ). To quantify fetal SPS, we created a novel index, the "SPS/DAO" ratio, measured as the ratio of the minimum infundibular diameter to descending aorta diameter (DAO) at the level of the diaphragm. Multiple linear regressions were used to predict post-natal PVZ from pre-natally determined parameters, including SPS/DAO. Fetal parameters were analysed by logistic regression for association with post-natal outcomes: timing of surgery (<I month) and type of surgery (trans-annular patch (TAP) vs. "valve-sparing").

Results: Twenty three fetuses met inclusion criteria. Mean gestational age was $21.8 \pm 1.9$ ( $16.6-25.4$ weeks). There was an excellent correlation between predicted and measured PV Z-scores, $r=0.82 \mathrm{p}<0.000 \mathrm{I}$, using the derived equation: $-3.68+0.9$ I* pre-natal PVZ - 4.44* SPS/DAO - 3.19 (pre-natal PVZ* SPS/DAO), A SPS:DAO value $<0.5$ had $100 \%$ sensitivity and $56 \%$ specificity for repair $<1$ month and $<0.47$ had $100 \%$ sensitivity and $75 \%$ specificity for TAP repair.

Conclusions: Postnatal PVZ can be predicted using pre-natal PVZ with the SPS/DAO ratio in $<26$ week fetuses with ToF. Quantification of SPS with the SPS/DAO ratio identifies patients who may require early intervention and/or TAP repair, thereby impacting pre-natal counselling. 